\section{Corps}

W........ers

Waterways Experiment

Station

\title{
Information Management for Installation Restoration with Focus on Aberdeen Proving Ground, Maryland
}

by Joe D. Manous, Jr.

U.S. Army Corps of Engineers

\author{
U.S. Army Corps of Engineers
}

Approved For Public Release; Distribution Is Unlimited

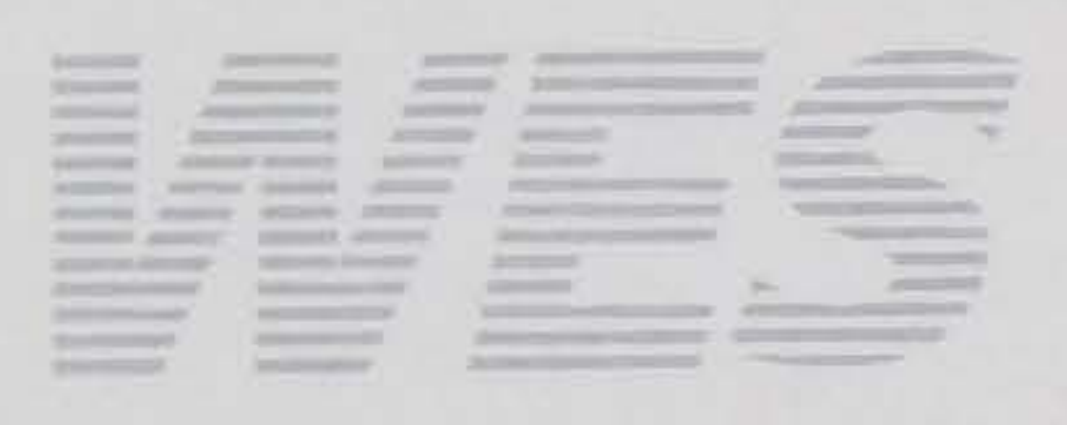

RESEARCH LIBRARY

US ARMY ENGINEER WATERWAYS

EXPERIMENT STATION

VICKSBURG, MISSISSIPPI

Prepared for Headquarters, U.S. Army Corps of Engineers 


\section{Information Management for Installation Restoration with Focus on Aberdeen Proving Ground, Maryland}

by Joe $\mathrm{D}$. Manous, Jr.

Department of the Army

U.S. Army Corps of Engineers

Washington, DC 20314-1000

Final report

Approved for public release; distribution is unlimited

Prepared for U.S. Army Corps of Engineers

Washington, DC 20314-1000

Monitored by Geotechnical Laboratory

U.S. Army Engineer Waterways Experiment Station

3909 Halls Ferry Road, Vicksburg, MS 39180-6199 


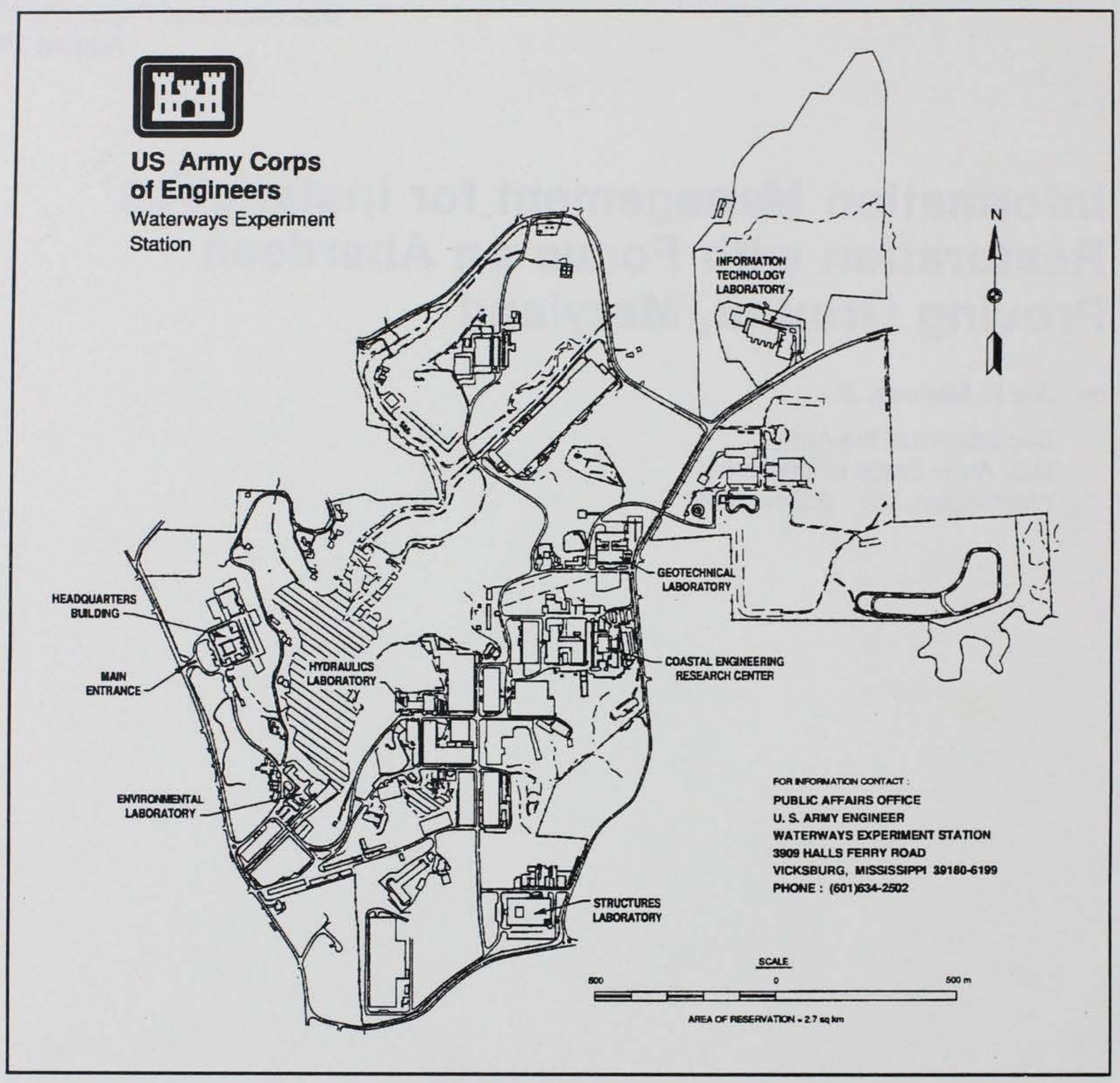

\section{Waterways Experiment Station Cataloging-in-Publication Data}

\section{Manous, Joe D.}

Information management for installation restoration with focus on Aberdeen Proving Ground, Maryland / by Joe D. Manous, Jr. ; prepared for U.S. Army Corps of Engineers ; monitored by Geotechnical Laboratory, U.S. Army Engineer Waterways Experiment Station.

65 p. : ill. ; $28 \mathrm{~cm}$. - (Miscellaneous paper ; GL-93-3) Includes bibliographical references.

1. Environmental engineering - Maryland - Aberdeen Proving Ground. 2. Data base management. 3. Environmental protection Data bases. 4. Geographic information systems. I. United States. Army. Corps of Engineers. II. U.S. Army Engineer Waterways Experiment Station. III. Title. IV. Series: Miscellaneous paper (U.S. Army Engineer Waterways Experiment Station) ; GL-93-3.

TA7 W34m no.GL-93-3 


\section{Contents}

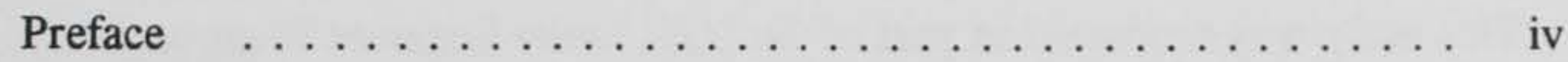

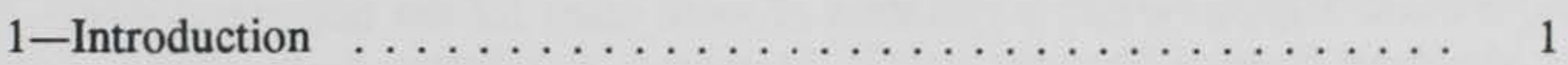

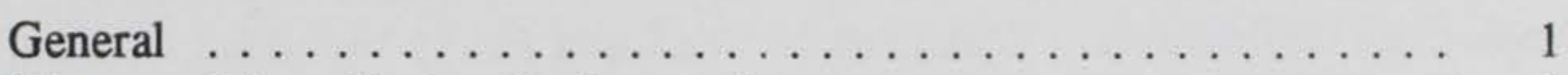

Edgewood Area Project Background $\ldots \ldots \ldots \ldots \ldots \ldots \ldots$

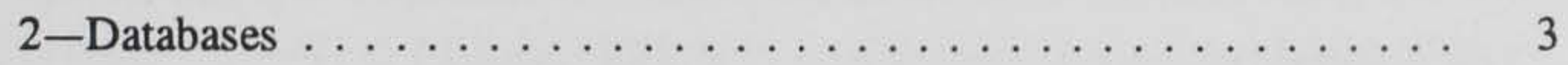

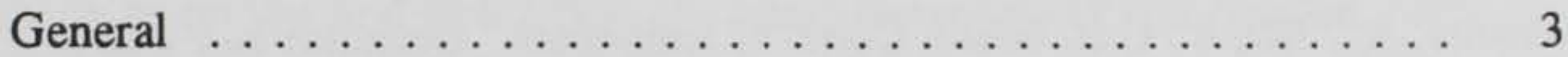

Database Requirements $\ldots \ldots \ldots \ldots \ldots \ldots \ldots \ldots$

Discussion ...................... 4

Services Provided with IRDMIS $\ldots \ldots \ldots \ldots \ldots \ldots \ldots \ldots$

Difficulties with IRDMIS $\ldots \ldots \ldots \ldots \ldots \ldots \ldots \ldots \ldots$

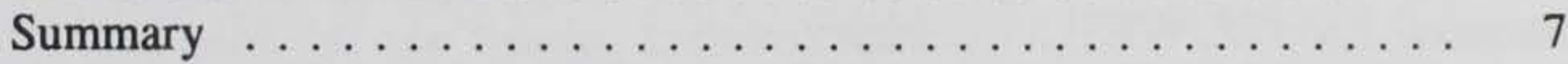

3-Geographic Information Systems $\ldots \ldots \ldots \ldots \ldots \ldots \ldots$

General . . . . . . . . . 8

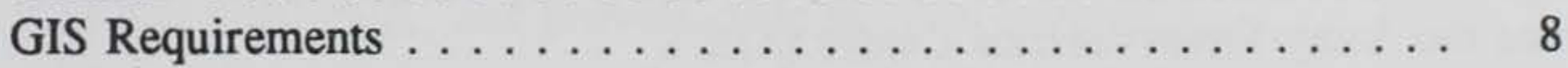

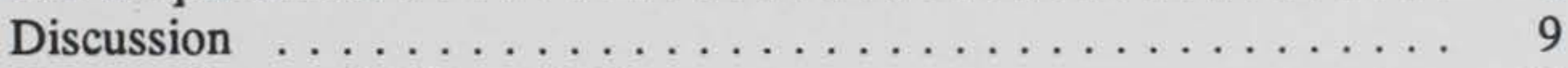

Current Status of GIS at APG $\ldots \ldots \ldots \ldots \ldots \ldots \ldots \ldots$

Specialty Graphical Systems $\ldots \ldots \ldots \ldots \ldots \ldots \ldots \ldots \ldots$

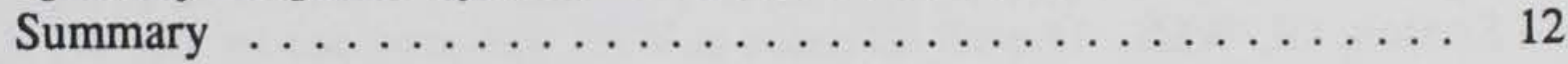

4-Recommendations . . . . . . . . . . . . . . . . . 14

Appendix A: Abbreviations $\ldots \ldots \ldots \ldots \ldots \ldots \ldots \ldots \ldots \ldots \ldots$

Appendix B: Addressees and Points of Contact $\ldots \ldots \ldots \ldots \ldots$ B1

Appendix C: Current THAMA Certified Labs $\ldots \ldots \ldots \ldots \ldots \ldots$ C1

Appendix D: IRDMIS General Information $\ldots \ldots \ldots \ldots \ldots \ldots$ D1

Appendix E: Condensed IRDMIS Data Dictionary $\ldots \ldots \ldots \ldots \ldots$ E1 


\section{Preface}

This study was conducted as part of the U.S. Army Engineer Waterways Experiment Station (WES) preparation of work plans for the Edgewood Area of Aberdeen Proving Grounds (APG), Maryland, Installation Restoration Project and Groundwater Contamination Studies at Rocky Mountain Arsenal, Colorado, during the period 2 June 91 to 17 July 91 .

The Principal Investigator and author of this report was Joe D. Manous, Jr., Major, U.S. Army Corps of Engineers. Graphics and Geographical Information System (GIS) technical support was provided by Mr. Gregory D. Comes, Earthquake Engineering and Seismology Branch (EEGD), Geotechnical Laboratory (GL-WES), and Mr. Mark Graves, Environmental Systems Division (ESD), Battlefield Environmental Group, Environmental Lab (EL-WES). Database technical support was provided by Ms. Benita Allen, Soil and Rock Mechanics Division, GL-WES and by Ms. Joann Pickett, Ms. Irene Vinsen, Ms. Laura Bremen, and Ms. Tracy Westbrook of Potomac Research, Incorporated (PRI) working under contract from the Army Environmental Center (AEC).

Direct supervision was provided by Dr. James H. May, Earthquake Engineering and Geophysics Division, EEGD, Hydrology and Site Characterization Section, GL-WES. Overall direction at WES was provided by Dr. W. F. Marcuson, III, Director, GL-WES.

At the time of publication of this report, Director of WES was Dr. Robert W. Whalin. Commander was COL Bruce K. Howard, EN. 


\section{Introduction}

\section{General}

This study reviews and evaluates database management systems currently used for chemical and geologic data storage, retrieval, and processing. A review was also conducted of Geographic Information Systems (GIS) and their use in coordination with different database programs and data formats. In addition to review and evaluation, the study consolidated information sufficient for inexperienced user access of the systems recommended by this study.

The focus for this study is the Edgewood Area (EA), of Aberdeen Proving Grounds (APG). In addition, consideration was given concerning applicability to Aberdeen Area of APG which will be concurrently remediated. In the larger context, the system evaluations performed should prove valid with respect to similar projects not associated with APG. The establishment of a standard information system is intended to yield increased economies of analysis time and techniques, and provide customer cost savings.

\section{Edgewood Area Project Background}

EA has been the site of extensive military munitions testing and disposal for over 70 years. Onsite burial of wastes was extensive until the 1970's. Some have been removed for remediation or "safer" storage. Unfortunately, much of the buried waste has not been recovered and no collective knowledge of burial sites is available.

The remediation process will require an extensive investigative effort to locate disposal sites and determine the extent of leachate movement. Additional information will be produced as the remediation process proceeds and understanding of the subsurface becomes better developed. The cumulative result will be an enormous body of information collected over the life of the remediation project. Remediation has been investigated and conducted at RMA for a period in excess of 17 years as of this writing. Storage of information for rapid accessibility is important as a base line for comparison of contaminant locations and concentrations over time and as a source of information whose importance may not be realized during the initial data review. 
Additionally, since long-term continuity of project remediation personnel is questionable, proper storage provides a means of "corporate memory" to prevent duplication of efforts. The method of storing and accessing chemical analysis and geotechnical data with the associated details of collection, handling, and analysis is the topic of this study.

Many environmentally related investigations at EA have taken place over the past 20 years. The results of these reports exist in paper copy and an assumed complete collection of these investigations is located in the offices of the EA, Director of Safety and Health (DSH). Producers of these reports include U.S. Geological Survey (USGS), Army Environmental Health Agency (AEHA), Environmental Protection Agency (EPA), WES, and private contractors. These reports are of variable usefulness and accuracy when compared with current AEC and EPA analysis standards. All reports, however, provide information useful from an investigative view point and may be the only historical records of a particular area. A portion of this information does exist in digital form in the Installation Restoration Data Management Information System (IRDMIS) operated by Army Environmental Center (AEC). 


\section{Databases}

\section{General}

An electronic database is a means of storing information for later sorting and retrieval. Input can be generated by typing at a keyboard or through electronic transfer in a standard format such as ASCII. Output is produced as tabular data printed in hard copy or as an electronic file. Graphical interfaces for database input or output were not part of this portion of the study.

\section{Database Requirements}

The following are specific requirements for a desired level of database functionality:

a. The database must be capable of handling large volumes of raw data or records either directly or through relational processes.

$b$. The database should be capable of importing and exporting information electronically using standard formatting procedures and in particular ASCII.

c. The database should be capable of performing user-specified searches and sorts of data.

d. The database system should be capable of producing user-specified reports suitable for presentation.

$e$. Setup and operating costs should be kept to a minimum. The intent is for a single, integrated database system.

f. The database should be easy to operate and not require specialized skills or an extensive training program.

g. The database system should operate on an existing computer system to reduce initial costs. (Not a problem at WES since computer options range from XT computers to supercomputers. 
The following are desirable criteria which aid in database use, but do not explicitly preclude a particular database system.

a. Predeveloped routines should be available for inexperienced users (i.e. a shell program). These routines should perform simple sorts and report production.

$b$. The system should be accessible by activities other than WES for information input and output. Concurrent with this criterion is the need for a database manager to oversee and maintain the database.

\section{Discussion}

During interviews with members of the EA-DSH, Baltimore District of the Corps of Engineers (a partner with WES in EA studies), the EPA, GL-WES, and EL-WES, no database system as outlined in paragraphs 7 and 8 was found in operation. Within these organizations, the most common general purpose database program was dBase.

AEC was the exception in information management. They have created a database specifically for the purpose of managing geotechnical and chemical analysis data under a program titled "Installation Restoration Data Management Information System" (IRDMIS). This program, begun in 1975, has undergone several updates as technology and database requirements have changed. The system is maintained for AEC under contract with Potomac Research, Inc. (PRI) and is physically collocated with AEC on EA, Maryland. Data from geotechnical chemical analysis and field survey results are supplied by AEC-authorized contractors and laboratories to PRI for input into the database. The system functions within a UNIX operating environment and uses Structured Query Language (SQL) as the database management format. SQL can be embedded in "C" or a proprietary formatting program called "Report-Writer" distributed by the IRDMIS computer and software manufacturer, Ingres. Both $\mathrm{C}$ and Report-Writer are currently available with IRDMIS.

Similar in operation to IRDMIS is a system employed at Rocky Mountain Arsenal (RMA), Colorado, by a contracted firm, D.P. Associates, Inc. That system also manufactured by Ingres is similar, but not as versatile as IRDMIS. A recent submittal by D.P. Associates has requested funding to upgrade to an IRDMIS equivalent software and hardware configuration.

RMA has used IRDMIS until 1985, but became disenchanted due to delays in processing information requests, database information integrity, duplicate entries, and data loss. Changes in hardware, software, and overall operation of IRDMIS have largely corrected the previous problems encountered by RMA. However, it is notable that even with the problems encountered with IRDMIS, RMA has chosen to stay with an IRDMIS compatible database system and continues to use AEC's Quality Assurance (QA) program. 
Using the data management systems currently available to activities involved at EA, a comparison evaluation was made between dBase and IRDMIS. Through a hands-on evaluation of these programs it was found that both adequately met the outlined database requirements (paragraph 7) with neither system showing any significant advantage or disadvantage.

In the desirable criteria area (paragraph 8), however, differences were apparent. A flexible user shell is possible for both systems and an IRDMIS shell currently exists. Changes, additions, and deletions to the IRDMIS shell must be justified, routed through AEC and placed in PRI's work schedule for action. This limits responsiveness to shell changes as could be performed in a locally operated system. An operator-defined shell could be installed within a user's directory on IRDMIS, but would not be directly supported by PRI (Academic Computing Division, USMA has such a UNIX based program). It should be noted that the greatest flexibility in database use is realized by running tailored query programs and not from a standardized shell interface. Neither dBase nor IRDMIS demonstrated a significant advantage in the use of tailored query programs.

In the second desirable criteria, a significant advantage of IRDMIS was apparent. The IRDMIS was designed and is managed to permit common access by many users for input and output. A similar input and output facility could be implemented using dBase, but would require a database manager such as PRI. Such a large and long-term commitment does not seem appropriate for GL's role at EA, nor is DSH-EA prepared to implement such a large scale project at this time. It should also be remembered that AEC provides the same database system for all Department of Defense (DoD) installations. Therefore, the IRDMIS skills and techniques employed at EA could be equally applied on similar projects at other federal installations.

\section{Services Provided with IRDMIS}

Several advantages and programs are available with IRDMIS to include program oversight by AEC and the availability of a dedicated database manager. AEC has made a long-term commitment to update and maintain the IRDMIS. How long is a matter of conjecture, but current indications are for long-term support.

AEC provides a QA program for chemical analysis labs supplying information. Tests from AEC certified labs (Appendix C) are characterized based on a combination of sampling techniques, sample holding times and other variables. The test results are then coded as to their accuracy and reliability. Data falling outside AEC-established criteria, not following AEC testing procedures, or coming from a non-AEC certified lab are coded "99." Much of the pre-1985 data in the IRDMIS is coded "99" because of current higher detection and handling standards. Unfortunately, test results from EPA's standard for chemical data collection, the Toxic Chemical Leachate Program (TCLP), are also coded "99." EPA TCLP data are a common, standardized 
testing procedure which can and should be incorporated in IRDMIS. An additional qualifying code could be added to the AEC coding list to indicate that the TCLP standard for chemical data collection and analysis has been followed. This inclusion should be pursued by EA-DSH and GL-WES.

In addition to chemical analysis QA, IRDMIS has a QA program for data integrity. All data submissions are reviewed by PRI to ensure that the data are properly identified and formatted. This check is concerned with qualitative entries and not with quantitative validity. An error such as omitting an installation identification code or using an undefined response would be identified as an error during the data QA check. On the other hand, a typographical error such as entering " 20 " instead of " 200 " $\mathrm{ft}^{1}$ for sample depth would not generate an error. The purpose of this check is to ensure sufficient information is provided to uniquely identify each record and maintain a minimum information level on each record. A MS-DOS program, "PC-Tool," was written and is maintained by PRI as the mechanism for data input. This menu-driven, interacting program checks data as they are entered for compatibility with the IRDMIS system. This is the same program used by PRI upon receipt of analytical data to again check for IRDMIS compatibility.

Chemical analysis data are not the only, nor the first entry into IRDMIS. Positional data (X, Y, Z locations) of analysis sites, wells, etc must be submitted prior to chemical analysis submissions. This process assures the completeness of the database record since the positional data and chemical data are produced by different sources. The positional data are also formatted for input using the program "PC-Tool." Universal Transverse Mercatur (UTM), longitude-latitude and state planar coordinate systems are honored. A brief summary of all database record entries can be found in Appendix E and a complete description is found in the IRDMIS Data Dictionary. No QA or QC program similar to AEC's lab certification is applied to positional data.

IRDMIS is also structured to record well construction information, logging results, and groundwater elevation data. As with any database, additional information types and records can be added as required. Again, a brief summary of all database record entries can be found in Appendix E and a complete description is found in the IRDMIS Data Dictionary.

Lastly, IRDMIS provides common user access. The IRDMIS is available to any authorized user through the Defense Data Network (DDN) or by modem. Connection details can be found in Appendix D.

1 To obtain meters, multiply feet by 0.3048 . 


\section{Difficulties with IRDMIS}

IRDMIS provides tremendous possibilities but is far from perfect. The major difficulty is the lack of user guidance and directions. No consolidated document or organization provides single source information concerning IRDMIS. Conceptual use and QA program questions are handled by AEC while specific hardware and software questions are handled by PRI. Input validation is physically accomplished by PRI, but AEC handles QA and sampling technique questions. Passwords are obtained through AEC, but connection details are handled through PRI. The representatives of AEC and PRI were found to be prompt and helpful with specific questions, but initial use required a personal visit to AEC and PRI along with substantial trial and error. Appendix D provides a consolidation of the basic information required for first time use of IRDMIS. Unfortunately, the lack of specific user guidance from a single source is a hindrance for potential users and will limit their desire to use this system.

As already stated, the presence of a contracted database manager provides significant advantages for this system. The presence of a contractor not directly responsible to the user also presents potential work prioritization problems. Though no difficulties were observed during this evaluation, specific requests for information, assistance, or service support could be delayed if AEC's or the PRI's work priority differs from the user.

\section{Summary}

In operability and function no significant difference was found between $\mathrm{dBase}$ and IRDMIS. IRDMIS provides the advantage of an established system with a dedicated database manager in place. IRDMIS also provides wide access of information by all investigative activities for most DoD sites in the United States. The use of IRDMIS will relinquish some user-control over data input as compared with a local database, but this loss should have a minimal impact on overall productivity. The major shortcomings of IRDMIS are insufficient documentation and added layers of management between the user and the data.

I recommend use of IRDMIS to store GL-WES IR data. The system is not perfect, but it is established and is capable of offering significant advantages in future IR work. I further recommend that EA-DSH contract an outside firm to review the investigative and remediation reports which have been collected for the EA. The pertinent data from each report can be reduced to digital form and submitted into IRDMIS. Finally, IRDMIS training sessions through AEC and PRI are available and should be attended by prospective IRDMIS users. 


\section{Geographic Information Systems}

\section{General}

A GIS is a means of graphically displaying land surface, geologic, chemical analysis, and so forth in a spatial or map-type format. Output is normally previewed on a computer screen with hard copy products available upon request. A GIS is capable of performing the same types of sorts and queries as a relational database though not with the ease, flexibility, or speed provided by a database program. In comparison with a database, a GIS exchanges speed of operation for graphical input and output capability. This speed tradeoff can be significant, but use of mainframe computers and recent advances in personal computers (PC's) has narrowed the difference.

\section{GIS Requirements}

The following are specific requirements required to satisfy this study's GIS objectives:

a. Functions:

(1) Sort by attribute name and by use of logical operators applied to attributes.

(2) Cross-sectional development capability (i.e. groundwater or geologic profiles).

(3) Ability to distinguish, display, and plot field entries in close proximity (i.e. well clusters $<10$-ft spacing).

(4) Able to print formatted output of all or selective tabular data chosen from the GIS interface.

(5) Able to plot scaled maps with a user selected grid system and user selected attributes. 
(6) Able to import data using $\mathrm{X}, \mathrm{Y}$, and $\mathrm{Z}$ coordinates as an import data field (direct input without digitizing).

b. Compatibility with common data formats:

(1) Import and export dBase files.

(2) Import and export ASCII files.

(3) Import and export INFORMIX files.

(4) Import and export Info files.

c. Operating system:

(1) Operate adequately on a "fast" PC (preferred operating system is MS-DOS, but this is not an absolute requirement).

(2) Able to shift system to a SUN or similar work station with minimal data conversions.

d. Cost:

(1) Minimal cost is always a major consideration. Development is based on two independent users, EA-DSH and GL-WES-preferably using available software and hardware.

e. Training:

(1) Considerable expertise will be required for system set-up and periodic system maintenance, but it is desirable that an "inexperienced user" interface be available for viewing common sorts, map plots, and tabular report generation.

\section{Discussion}

Unlike databases, a standard GIS has not evolved in installation restoration work. EA-DSH, the Corps of Engineers Baltimore District, U.S. EPA and RMA do not currently employ a GIS. EL-WES is working in ARCInfo and GL-WES has people trained and platforms available to operate ARCInfo, CAMMS, and Intergraph. Numerous Corps of Engineers District offices use Intergraph as their GIS. GRASS is widely used on U.S. military installations around the world in conjunction with the Installation Training and Management System (ITAMS). Finally, RMA has developed an elaborate computeraided drawing (CAD) system, which is used in conjunction with a database to produce products similar to a GIS. 
The following GIS systems were considered during this study; ARCInfo, Intergraph, CAMMS and GRASS. All systems could meet the functions and compatibility requirements of paragraph 27 . However, ARCInfo and Intergraph met the functions requirements with the greatest ease, and ARCInfo had a distinct advantage in compatibility over all four systems. GRASS, a raster GIS, has difficulty distinguishing features in close proximity, however, vector overlays can be produced to overcome this obstacle. CAMMS required some software improvements to meet all of the requirements in paragraph 26 .

Costs of GIS's ranged from extreme to no expense. Intergraph is the most expensive since all software and hardware is proprietary. ARCInfo is moderately priced and will run on most UNIX based work stations such as a SUN or a mainframe such as a VAX. A PC version of ARCInfo is now available and operates under MS-DOS using dBase files for relational data storage. This system best operates on a "fast" PC and is upwardly compatible with work station and mainframe versions of ARCInfo. GRASS software and technical support is available at no cost from the Construction Engineering Research Laboratory (CERL). GRASS operates in a UNIX environment, normally on a work station. Finally, CAMMS GIS software and limited technical support is available at no cost from the Mobility Section, GL-WES and will run on a PC.

\section{Current Status of GIS at APG}

The EL-WES has recently completed digitizing the man-made and natural features of EA. The digitized database is a compilation of different map series over the past 40 years. This work was performed on a reimbursable basis for the EA-DSH. The project includes digitization, selection of a GIS (PC version of ARCInfo) and procurement of a hardware system to support the software. During the study period, EA-DSH was not proficient in the use of ARCInfo, but is scheduled for training by EL-WES. Upon approval of the EA work, EL-WES will begin a similar digitization of the Aberdeen Area of APG.

Until completion of EL's digitization of EA, no single map series adequately represented EA. Many of the map series used in digitization were based on single coordinate systems and required conversions between longitude-latitude, state planar, or local coordinate systems into Universal Transverse Mercatur (UTM) coordinates. The standard coordinate system for the completed GIS is UTM. The conversions along with inadequate survey control of some large scale maps have introduced an as yet undetermined error in positional representations. The GIS is generally a better source of information than previously available, but will require validation by ground survey before GIS products should be used in final IR assessments or in-depth development of IR work plans. Use of a global positioning system (GPS) would be ideal for the validation work. 


\section{Specialty Graphical Systems}

CAD represents another approach in spatial information representation. CAD programs such as AutoCAD are common through Corps of Engineer activities to include GL-WES. These programs usually operate on "fast" PC's and provide, quality two-dimensional (2-D) and limited three-dimensional (3-D) mapping and graphic display capability. CAD programs are easily manipulated and are ideal for one of a kind projects. RMA has expanded on the CAD concept and developed a detailed CAD installation map which is managed by a private contractor. By using "layers" of information, similar to an acetate map overlay, details can be added to a base map. The result is a quality, scaled drawing. Unfortunately, CAD additions and deletions must be performed manually, often at considerable expense in time and money. A GIS is advantageous because it can quickly create new overlays by querying for desired features or attributes and then generating overlays internally. The advantage of GIS's increases as the areas under study become larger or more detailed. The contractor responsible for information management at RMA, DP Associates, has recently submitted a proposal for purchase of an ARCInfo system to transition RMA from CAD to GIS.

Graphic programs are another area of interest in spatial information displays. Most graphics programs are not GIS oriented, nor do they have the drawing flexibility of CAD. Many of these programs are aimed at interpolation of data sets and developing lines of equal concentrations, elevations, etc. This process, contouring, is a "best fit" process requiring the use of various polynomial and regression techniques applied in a trial and error fashion. These techniques can be used in analysis, but commonly the use is simply information exchange. The construction of 3-D graphical models can be a tremendous asset in conveying a concept or perception. In addition to plan, perspective, isometric or similar views, such programs also have crosssectional capabilities which can be useful in displaying geologic profiles or contaminant plumes.

A graphical program available through AEC is Interactive Surface Modeling (ISM) developed by Dynamic Graphics. This program is accessed by telnet or modem on THAMA3 and THAMA6 logins. Though the program can be executed from any PC or equivalent terminal, a "graphics terminal, such as a Tectronix or PC with Tectronix emulation software, is required to view the plotted results on screen. The plots can be stored in a data file for later retrieval and local printing. Input for ISM is generated from reports written from IRDMIS. Standard reports exist in the IRDMIS IR menu, but tailored reports can also be written, as discussed in Part II of this report. Once generated, contaminant contours, groundwater elevations, etc can be plotted, contoured, and displayed in 2-D or 3-D.

A complete Iris work station with ISM is available at GL-WES. This system can be linked directly with IRDMIS by telnet to provide faster (local) compilation of data than remote access. Direct linkage of the GL-WES Iris to a plotter is also available. 
Another common, PC based, graphic program is SURFER. Though not as fast nor elaborate as ISM, SURFER is a fully capable, 3-D graphics package which can display surface topography or similar information such as surface or groundwater levels. These displays can be viewed in 2-D as plan, contoured views, or in 3-D as perspective views. SURFER is commonly used in conjunction with CAD programs such as AutoCAD.

A recent development in graphical displays is VHS video presentations. WES has been working with video presentations which consist of multiple computer generated section and perspective views appended similar to frames of a cartoon. The result is a dynamic visual presentation offering dynamic views from several perspectives. A commercial firm, Z-Axis of Aurora, Colorado, produces similar videos, and can use animation technology to fill gaps between successive computer generated views. The application of animation technology is intended to reduce the number of required computer generated views and presumably lower production costs. Similar products of both animation and computer simulation can be produced at ITL-WES. These technologies are new, still developing and relatively expensive. The animation technology claims a savings in computational expense and development time, however, insufficient information was available to validate that claim in this study.

\section{Summary}

All of the GIS's or combination of CAD and database programs reviewed were capable of meeting the requirements as described in paragraph 27 (CAMMS would require some software enhancement). Since EA-DSH has already purchased an ARCInfo system and a digitized database through EL-WES, there is no technical reason for EA-DSH or GL-WES to implement a supplemental GIS. A copy of the ARCInfo database can be obtained from EL-WES. There is no cost involved with GL-WES operating the database on an existing ARCInfo platform. However, an additional user fee will be required for each copy of the PC version of ARCInfo obtained.

The use of a CAD program may be desirable for an individual investigator working at EA. However, a well maintained GIS should support most user's needs. A centralized approach to GIS will reduce effort and cost duplications, and provide each user with the same current information.

ISM is available at WES and through AEC. The incorporation of the data sets from the EA ARCInfo database was possible and was completed for buildings, roads, shorelines, wetlands and elevation contours as part of this study. Unfortunately, the ability to construct an adequate "gridding" file of the contour data does not currently exist. The "gridding" file is the first step in data interpolation required for 3-D graphic development. The developer of ISM, Dynamic Graphics, has been notified of this software shortcoming and is currently working on a solution. 
IRDMIS data are compatible with the ARCInfo database. As part of this study, a copy of EA analytical and positional well data was downloaded from IRDMIS, converted to dBase format, and provided to EL-WES for incorporation in the ARCInfo data set they are creating for EA.

Finally, the owner of the GIS, EA-DSH, needs to provide a mechanism for the validation of the digitized data and correction of errors as they are discovered. In addition, multi-user access to the GIS and a method of producing user requested GIS products needs implementation. These services can be provided by a dedicated "in-house" GIS manager or through a contractor. 


\section{Recommendations}

An installation policy needs implementing concerning the storage of IR analytical and geologic data for APG. This policy should be applicable to all IR work performed at APG. A unique database could be developed, but the collocation of AEC at APG and the experience of that organization with IRDMIS make new database development an unnecessary duplication of effort and cost. Designation of a mandatory data storage procedure by APG would require the establishment of direct and active communication between APG and AEC concerning IRDMIS. This interaction would be essential to ensure the needs of APG are met and supported.

In the absence of an installation directed policy concerning the storage of IR data, encouragement should be provided by DSH-EA for submittal of IR data produced at EA into IRDMIS. Whether specifically supportive of the APG IR program or not, IRDMIS is still the best available long-term repository of this information. IRDMIS data submission should be accomplished regardless of whether the information producer intends to access the data through IRDMIS or obtain it directly from an analysis lab. Reasons for IRDMIS submittal are two-fold. First, the data are part of an irreplaceable historical record, and second all data should be commonly available to each contractor and investigator working at APG. As the volume of acquired data increases over the next 10-15 years electronic access and retrieval will become essential to completely review all of the data produced. It is acknowledged that the use of IRDMIS will increase the cost of analysis processing and delay the return of analytical data (unless duplicate reports are requested for the user and AEC, which bypasses AEC's internal QA process). The long-term benefits, however, should outweigh these short-term costs.

When possible, utilize IRDMIS directly for data queries, retrieval, and development of data reports. The system is available and with use can be as easy to implement as $\mathrm{dBase}$.

The EA ARCInfo database should be validated by ground survey and/or GPS and corrected as necessary. IRDMIS well positions within the same area should be included in the validation. This should be a short-term objective.

APG should implement a full-time GIS manager responsible for EA and Aberdeen Area. This person(s) can be in-house or contracted. The utility of a GIS is directly related to two factors. First, the data must be accurate (as 
stated above) and second there must be "real time" interaction between GIS client requests and GIS output. Without the successful accomplishment of both factors, the credibility of the GIS will suffer and its full potential will not develop.

Work should continue to transfer all EA ARCInfo data into an ISM compatible format. Contact with Dynamic Graphics should be maintained concerned the transfer of the digitized ARCInfo contour data into a suitable "gridding" file. This development does not hinder the addition of contaminate data into ISM, but does limit the comparison of such data with respect to the topographic surface.

APG should instigate changes through AEC concerning the incorporation of TCLP data into the IRDMIS. If the required data standard for EA remains TCLP analysis, then consideration for coding this information should be provided within IRDMIS.

EA-DSH should reduce the on-hand hard copy reports of investigative and remediation work at EA into a digital format for incorporation in IRDMIS.

This work can be accomplished in-house or by contract. The information may not be of litigation quality but is an important source of historical and investigative information if placed in a format conducive to rapid query and retrieval. 


\title{
Appendix A Abbreviations
}

\author{
AA Aberdeen Area, Aberdeen Proving Grounds, MD \\ AEC Army Environmental Center \\ AEHA Army Environmental Health Agency \\ APG Aberdeen Proving Grounds, MD \\ ASCII American Standard Code Information Exchange \\ CAD Computer Aided Drawing \\ CLP Chemical Leachate Program \\ DSH Director of Safety and Health, EA APG
}

EA Edgewood Area, Aberdeen Proving Grounds, MD

EL-WES Environmental Laboratory, Waterways Experiment Station

EPA U.S. Environmental Protection Agency

GL-WES Geotechnical Laboratory, Waterways Experiment Station

IR Installation Restoration

IRDMIS Installation Restoration Data Management Information System

ISM Interactive Surface Modeling computer program

ITL-WES Information Technology Laboratory, Waterways Experiment Station

PRI Potomac Research, Incorporated

QS Quality Assurance 
QC Quality Control

RMA Rocky Mountain Arsenal, CO

USGS U.S. Geological Survey

WES Waterways Experiment Station, Vicksburg, MS 


\section{Appendix B \\ Addresses and Points of Contact}

1. Dynamic Graphics - Address: Dynamic Graphic, Inc. 1015 Atlantic Avenue Alameda, CA 94501

Technical and Sales Information (415) 522-0700

2. Grafpoint - Mailing Address: Grafpoint, Inc. 1485 Saratoga Avenue

San Jose, CA 95129

Sales Representative

Mr. Roy Caudill, (408) 446-1919, FAX (408) 466-0666

3. Ingres Corporation - Address: Ingres Corporation Marina Village Parkway

Alameda, CA 94501

Sales Representative

Mr. Tom Baldwin, (415) 748-2519, FAX (415) 748-2545

4. PRI - Mailing Address: Potomac Research, Inc.

P.O. Box 14

Gunpowder Br.

Aberdeen Proving Grounds,

MD 21010

\section{Program Manager}

Mr. Warren J. Wortman, (301) 679-3030, FAX (301) 676-0802

Database Administrator

Ms. Irene Vinsen, (301) 679-3030, FAX (301) 676-0802 
5. RMA - Mailing Address: (Installation Contractor for Data Management)
DP Associates

Rocky Mountain Arsenal

Building 111

Commerce City, CO 80022

Regional Manager

Dr. Jack C. Pantleo, (303) 287-3231

6. AEC - Mailing Address:

USAEC

ATTN:

Aberdeen Proving Grounds, MD 21010-5401

Edgewood Area, APG Data Management Supervisor

Ms. Roxann Moran, (301) 671-1544, FAX (301) 671-1548

AEC Chemistry Branch, EA Project Officer

Mr. Doug Stevenson, (301) 671-3348

AEC Geological Branch (Also past use of ISM with IRDMIS)

Mr. Ira May, (301) 671-1516

7. WES - Mailing Address:

\author{
USAE-WES \\ ATTN: CEWES- - (Name) \\ 3909 Halls Ferry Road \\ Vicksburg, MS 39180-6199
}

Report Supervisor, GL (CEWES-GG-YH)

Dr. James H. May, (601) 634-3395, FAX (601) 634-3453/3139

Silicon Graphics Use, GL (CEWES-GG-H)

Mr. Gregory D. Comes, (601) 634-3395, FAX (601) 634-3453/3139

ARCInfo based GIS Production of APG, EL (CEWES-EN-B)

Mr. Mark Graves, (601) 634-3395

8. Z-Axis Corporation:

Z-Axis

(Video Graphic Production)

10800 E. Bethany Drive

Suite 500

Aurora, CO 80014

Vice-Present

Mr. Raymond C. Hauschel, (303) 696-9608, FAX (303) 696-0857 
9. Study Investigator:

CPT Joe Manous

Department of Geography and

Environment Engineering

United States Military Academy

West Point, NY 10996

(914) 938-2472, FAX (914) 938-4175 


\section{Appendix C Current AEC Certified Labs}

Arthur D. Little, Inc.

California Analytical Laboratory, Sacramento, CA

Environmental Science and Engineering, Denver, CO

Environmental Testing and Certification, Edison, NJ

EA Engineering Science and Technology

Interpoll, Inc., Circle Pines, MN

International Technologies Corp., Knoxville, TN

Midwest Research Institute, Kansas City, MO

Pace Laboratories, Inc., Minneapolis, MN

Rocky Mountain Analytical Laboratory, Arvada, CO

Rocky Mountain Arsenal Laboratory

Datachem (Utah Biomedical Testing Laboratory)

Roy F. Weston, Lionville, PA

Roy F. Weston, Stockton, CA

Radian Corporation

VERSAR

NOTE: Some labs are not certified for the full range of AEC specified procedures. 


\section{Appendix D IRDMIS General Information}

IRDMIS (Installation Restoration Data Management Information System) is the current product of a 15-yr effort by AEC to develop a data management system. IRDMIS is currently managed by a contractor, Potomic Research Institute (PRI). PRI and AEC are collocated at Edgewood Area, APG (See Appendix B for POC's).

IRDMIS is a relational database operating on a Pyramid computer within a UNIX operating system. The database is a product of the Ingres Corporation and software support to included programming manuals is provided through that firm (See Appendix B).

IRDMIS can be accessed by either telnet or modem.

a. The telnet and FTP address for the "THAMA1" system is 131.92.80.11

b. IRDMIS can be reached by modem using VT-100 emulation at:

(301) 671-4550, 300-2400 baud Hayes compatible (301) $671-4650,300-1200$ baud Hayes compatible (301) 671-4750, 9600 baud Telcor

All modem connections must be made initially as a subscriber to THAMA1.

(1) Crosstalk communications software use: Even Parity; 8 Data Bits; 1 Stop Bit

(2) Procomm communications software use: Even Parity; 7 Data Bits; 1 Stop Bit

c. Connection to other THAMAx systems can be made by telnet from THAMA1 by using "telnet THAMAx," where " $x$ " is the name of the system being connected. 
d. THAMA1 and THAMA3 permit access to the IR database. THAMA3 also provides access to the ISM program developed by Dynamic Graphics. Each THAMAx system requires a separate login and password.

Logins are obtained through the AEC area representative. For EA, the representative is Ms. Roxann Moran (See Appendix B). A login application form is available in Enclosure 1. Access to more than one THAMAx system must be annotated separately on the application.

Upon login, a menu of available report formats can be displayed by typing "IR" \{return\}. The Installation Remediation Menu of report formats will be displayed. These reports, used in conjunction with the IRDMIS data dictionary, are relatively easy to manipulate but are inflexible in their structure of queries and output. Greater flexibility can be obtained by writing specific queries in the system's database language, SQL (Structured Query Language). To implement SQL, the SQL code must be imbedded in another programming language such as $\mathrm{C}$ or FORTRAN. Provided by Ingres is an executable and formatting code called "Report-Writer." Report-Writer is similar to FORTRAN in usage.

IRDMIS data management is broken into three levels.

a. Level 1 data - Input data provided from a lab or other source. Analytical data which meet AEC certification must be analyzed and submitted from a AEC certified lab (Appendix B). Survey and positional data (required for each analytical submission) are provided by the crew obtaining the sample or an independent survey crew. In all cases, the data are placed into the appropriate digital format by the submitter using "PC Tool" or other programs which produces output compatible with PC Tool. PC Tool was produced and is maintained by PRI. Submission of analytical data not meeting AEC certification (including EPA TCLP) requires direct coordination with the local AEC data management supervisor.

b. Level 2 data - Data processing within the IRDMIS system. End users have no interaction with this data level.

c. Level 3 data - Output data which are accessible using SQL. Details of codes, record names, tables etc. are available in the IRDMIS data dictionary. A condensed version of the data dictionary is available in Appendix E.

A PC program called "PC Link" is available to connect a PC with an Ingres database. PC Link permits direct conversion of database information into other data format types such as dBase or Lotus. PC Link is available from the Ingres Corporation.

Interactive Surface Modeling (ISM) is a software program developed by Dynamic Graphics. This program is available for remote use on the THAMA3 and THAMA6 logins. Input for ISM can be generated from 
standard query reports available from the IR User Menu or tailored query reports generated with SQL. The input format is ASCII. ISM provides spatial plotting capability for the tabular data generated from the IR database. The data can be contoured and/or displayed in 3-D perspective presentations. The results can be viewed on screen or sent to a hard copy printing device using HPGL graphic output. Viewing on screen requires a graphic terminal such as an Iris work station, Tectronix terminal, or use of a graphic terminal emulation package on a PC. One possible emulation package is marked by Graphpoint, Inc. In addition to plotted data, annotation files (roads, elevation contours, water, etc) can be produced to enhance the visual interpretation of the plotted data. Annotation files are not part of the contouring or 3-D development and are used only for presentation enhancement.

Documentation available concerning the use of IRDMIS and associated utilities is as follows:

a. Ingres/Reports: Report-Writer Reference Manual, release 6.3, November 1989.

Available from: Ingres Corp.

Cost: $\$ 25.00$

GSA Contract GS00K91AGS5822

b. Ingres/SQL Reference Manual

Available from: Ingres Corp.

Cost: $\$ 55.00$

GSA Contract GS00K91AGS5822

c. USATHAMA Quality Assurance Program, USATHAMA PAM 11-41, January 1990.

Available from: AEC

Cost: No Charge

d. USATHAMA User's Guide, produced by PRI, November 1989.

Available from: AEC

Cost: No Charge

e. THAMA User's Manual, PC Data Entry and Validation Subsystem (IRDMIS PC Tool), version 4.2, produced by PRI, April 1991.

Available from: AEC

Cost: No Charge

f. PC Tool Software, version 4.2, produced by PRI.

Available from: AEC

Cost: No Charge 
g. THAMA User's Manual, Data Dictionary, version 1991.2, produced by PRI, April 1991.

Available from: AEC

Cost: No Charge

h. PC Link Software

Available from: Ingres Corp.

Cost: $\$ 130.00$

GSA Contract GS00K91AGS5822

i. Grafpoint Emulation Software

Available from: Grafpoint, Inc

Cost: $\$ 746.25$

GSA Contract GS00K90AG55259PS01 


\section{Appendix E Condensed IRDMIS Data Dictionary}

Pages E-2 and E-3 contain a two-page summary of key level 3 data record descriptions contained in IRDMIS. This summary is sufficient for the beginning user to extract information from IRDMIS using either the existing IR MENU or tailored queries using SQL. See the IRDMIS Data Dictionary for a detailed listing of IRDMIS data record descriptions.

Page E-4 is a current listing of data tables contained in IRDMIS. Also annotated are field names contained in each table and the key fields required for relating different tables during queries.

Page E-5 through E-35 contain a selected extract of the IRDMIS Data Dictionary.

The complete IRDMIS Data Dictionary is available through AEC. See Appendix D for details. 


\section{CODE SUMMARY SAFET}

for

The Installation Restoration Data Management Information System (IRDMIS) System Owner - Toxic and Hazardous Materials Agency (THAMA) System Operator - Potomac Research, Inc (PRI)

1. Installation code (inst) - Identifies installation from which the data were collected.

Common Examples: $\quad A A=$ Aberdeen Area, Aberdeen Proving Ground, Md

$\mathrm{CR}=$ Crane Naval Weapons Support Center, IN

$\mathrm{EA}=$ Edgewood Area, Aberdeen Proving Ground, Md

$\mathrm{RK}=$ Rocky Mountain Arsenal, CO (data after 1984)

RM $=$ Rocky Mountain Arsenal

2. Eile Type or Media Type (media_type) - Code identifying the type of data.

Common Examples: $\quad$ CGW = Chemical Ground Water

(Currently the $\quad \mathrm{CSW}=$ Chemical Surface Water

only terms in $\quad \mathrm{CSE}=$ Chemical sediment

use)

cSO = Chemical Soil

3. Site Type (site_type) - Represents a type of landmark, feature or construction.

Common Examples:

$$
\begin{aligned}
& \text { FBLK }=\text { Field Blank } \\
& \text { FELD }=\text { Field } \\
& \text { SPTK }=\text { Septic Tank } \\
& \text { SUMP }=\text { Sump }
\end{aligned}
$$

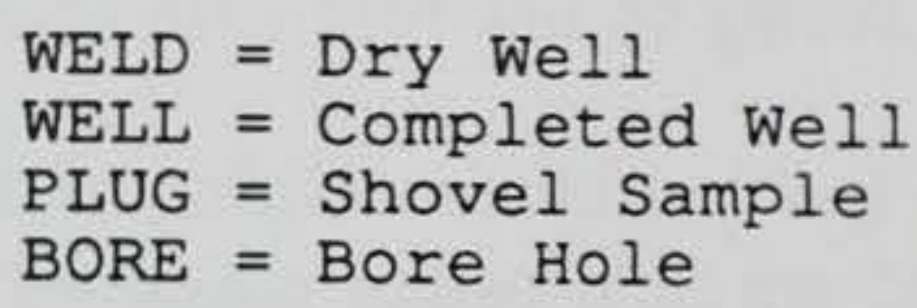

4. Depth (depth) - Depth to the nearest foot from the topographic surface to the interval being sampled. [-9999.0 is used to indicate no data was recorded, since an entry of 0 is possible. Well locations ( $x, y, \& z$ coordinates) are relative to a local datum].

5. Sample Date (samp_date) - Date sample was taken in the field. The date of actual testing of the sample (anly_date) is also available.

6. Analysis Type (anly_type) - Code representing the certification level of the analysis.

Common Examples: C1, 1A, 1B, \& C2 can all indicate a competent analysis level. (see data dictionary for details)

$$
\begin{aligned}
& 00=\text { Analytes not requiring certification } \\
& 99=\text { Quality level of analysis unknown or very poor }
\end{aligned}
$$

7. Analysis Accuracy (anly_acc) - Decimal number representing the standard error of the best-fit linear regression line of Found vs Target values for QC standard additions data.

8. Value (value) - Numerical value of analysis result (6 digit floating decimal precision). 
9. Measurement Boolean (meas bool) - Indicator that a measured quantity is not within the certified range, or that the test used does not yield quantitative results.

Common Examples: $\quad E Q=$ Equal to certified reporting or detection limit

$\mathrm{LT}=<$ Certified reporting or detection limit.

$\mathrm{GT}=>$ Certified reporting or detection limit.

blank = Within acceptable range.

ND $=$ Not Detectable.

10. Unit of Measurement (unit_meas) - Units of measured value.

Common Examples: $\quad$ UGL $=$ micrograms/liter

UGG = micrograms/gram

$\mathrm{PPM}=$ parts/million

11. Elagging code (i_s_c) - Code to indicate other-than-usual conditions or results.

Common Examples: $\quad \mathrm{D}=$ Duplicate sample or test name.

$\mathrm{E}=$ Element run with background corrections.

$\mathrm{H}=$ Out of control, but data accepted due to high recoveries.

blank = No special conditions apply to the results.

12. Rrime contractor (lab prime) - organization conducting or orchestrating a given data collection action.

Common Examples: $\quad A L=$ Arthur D. Little

$\mathrm{TH}=\mathrm{THAMA}$

$\mathrm{AH}=$ Army Environmental Hygiene Agency (AEHA)

GS = US Geological Survey

$\mathrm{WE}=\mathrm{WES}$

13. Test Name (analyte) - Parameter being measured.

Common Examples:

$11 \mathrm{DCE}=1,1-\mathrm{Dichloroethylene}$

$111 \mathrm{TCE}=1,1,1-\mathrm{Trichloroethane}$

$12 \mathrm{DCLE}=1,2-\mathrm{Dichloroethane}$

AS $=$ Arsenic

$\mathrm{C} 6 \mathrm{H} 6=$ Benzine

$\mathrm{CD} \quad=$ Cadmium

CMONOX = Carbon Tetrachloride

CLDEN = Chloride

$\mathrm{CR} \quad=$ Chromium

$\mathrm{CU} \quad=$ Copper

ENDRN = Endrin

$\mathrm{FE} \quad=$ Iron

$\mathrm{PB} \quad=$ Lead

LIN = Lindane

MN $=$ Manganese

$\begin{array}{ll}\text { HG } & \text { Mercury } \\ \text { MEXCLR } & =\text { Methoxychlor } \\ \text { NO3 } & =\text { Nitrate } \\ \text { PCB1016 } & \text { PCB } 1016 \quad \text { (etc) } \\ \text { PH } & \text { pH } \\ \text { SE } & =\text { Selenium } \\ \text { AG } & \text { Silver } \\ \text { STYR } & \text { Styrene } \\ \text { SO4 } & \text { Sulfate } \\ \text { MEC6H5 } & \text { Toluene } \\ \text { TXPHEN } & \text { Toxaphene } \\ \text { TRCLE } & =\text { Trichloroethylene } \\ \text { C2H3CL } & \text { Vinyl Chloride } \\ \text { XYLEN } & \text { Xylenes } \\ \text { ZN } & =\text { Zinc }\end{array}$


LEVEL 3 FILE FORMATS

installation
IRDMIS Level 3 Data Record Tables

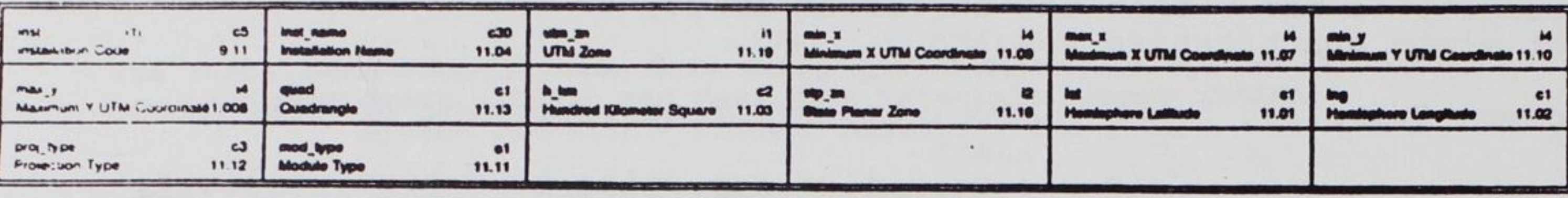

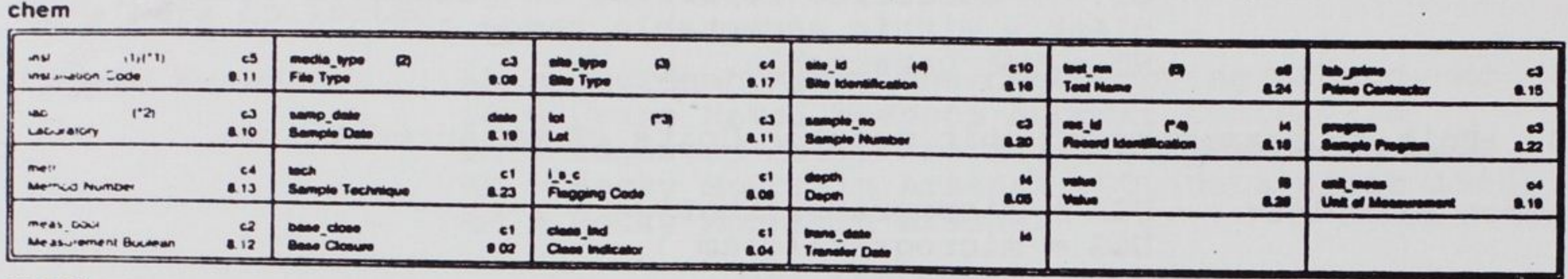

\section{chem2}

\begin{tabular}{|c|c|c|c|c|c|c|c|c|c|c|}
\hline 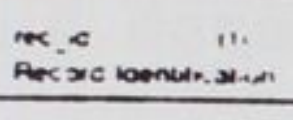 & 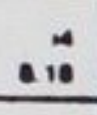 & Find & $\underset{0}{\infty}$ & Dom & 0.21 & 我 & $\infty$ & : & and & 20. \\
\hline 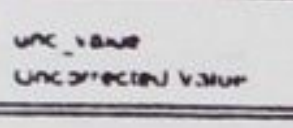 & .20 & anden factor & $\infty$ & mind & 214 & Tipos & $\infty$ & $=$ & & \\
\hline
\end{tabular}

sample_loc

\begin{tabular}{|c|c|c|c|c|c|c|c|c|c|c|c|}
\hline ns: & $\begin{array}{l}\infty \\
011\end{array}$ & $\sin _{1 \infty \pi \infty}^{\infty} \infty$ & a 17 & $=10$ & $\begin{array}{l}\mathbf{1 0} \\
010 \\
\end{array}$ & Dimes & $\infty$ & 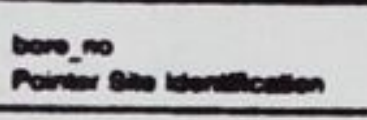 & $\begin{array}{l}210 \\
213 \\
\end{array}$ & $\min$ & 201 \\
\hline$\because x$ & 0 & 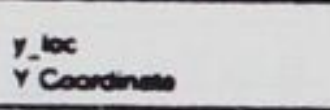 & .21 & 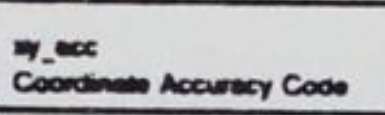 & $\stackrel{2}{a}$ & 2loce & 210 & Xex & $\underbrace{0}_{200}$ & 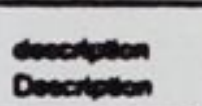 & $\begin{array}{c}\text { ese } \\
\text { 207 }\end{array}$ \\
\hline 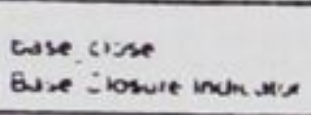 & $\stackrel{c 1}{9 \infty}$ & 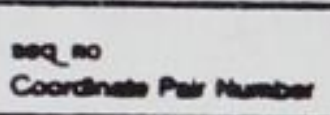 & 20 & & & & & & & & \\
\hline
\end{tabular}

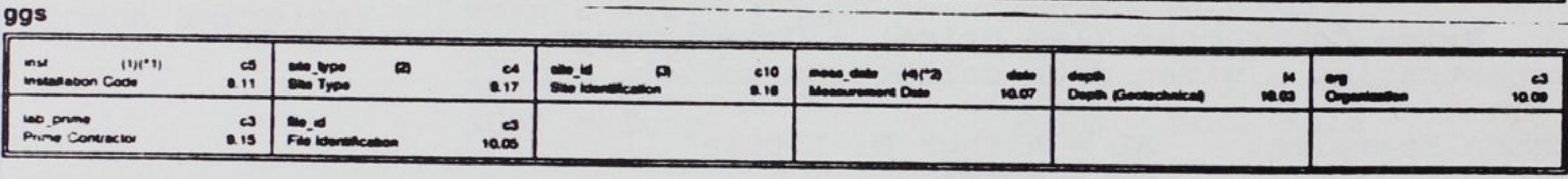

gwc

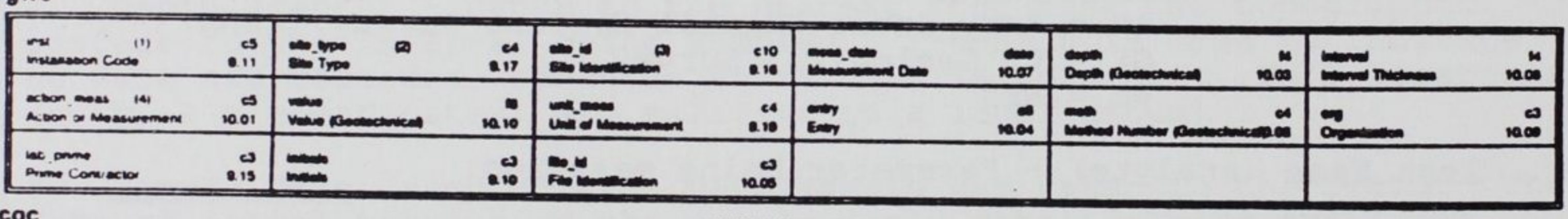

cqc

\begin{tabular}{|c|c|c|c|c|c|c|c|c|c|c|}
\hline invereson cosos" & an & Fin & $\underset{\infty}{\infty}$ & $\infty$ & an & $\infty \cos$ & an & $\infty$ & $\infty$ & $\underset{200}{\infty}$ \\
\hline 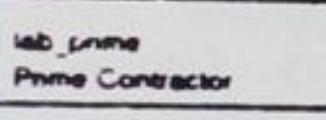 & ais & ind & $\infty$ & $=$ & $\underset{200}{10}$ & Lat & $\infty$ & $\infty$ & minnes & $\underset{20}{\infty}$ \\
\hline monos nemoser & ais & 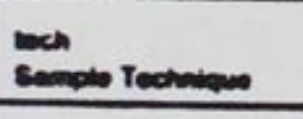 & et & 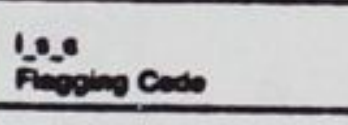 & $\begin{array}{c}c 1 \\
0\end{array}$ & $\infty$ & $\stackrel{m}{\infty}$ & $\infty$ & indm & $\underset{a 10}{\infty}$ \\
\hline 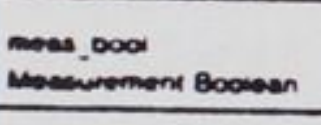 & $\begin{array}{r}0 \\
212 \\
\end{array}$ & 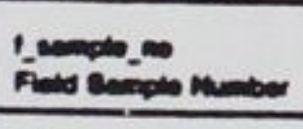 & eक & 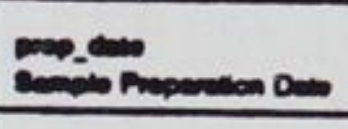 & 21 & indinam & $\infty$ & in & 舟 & 201 \\
\hline unceriecioso value & .20 & oted foctor & 200 & Dat & 214 & 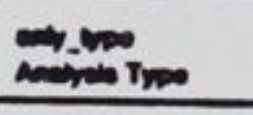 & $\infty$ & - & $\infty$ & act \\
\hline mas: & $\begin{array}{r}c 3 \\
0.10 \\
\end{array}$ & mand & in & & & & & & & \\
\hline
\end{tabular}

gid

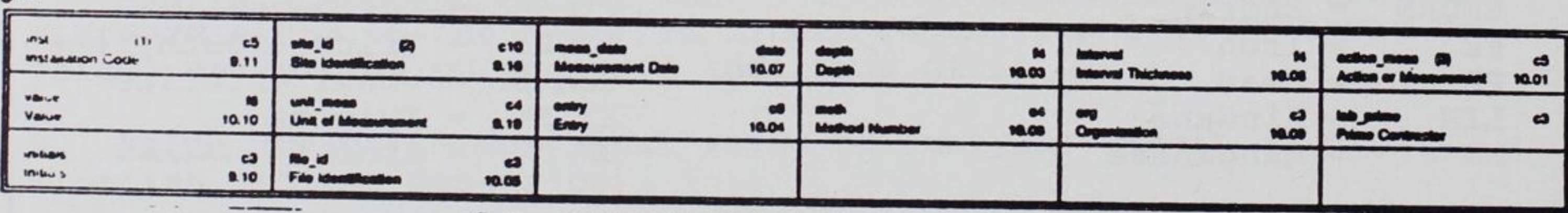

IV12_log

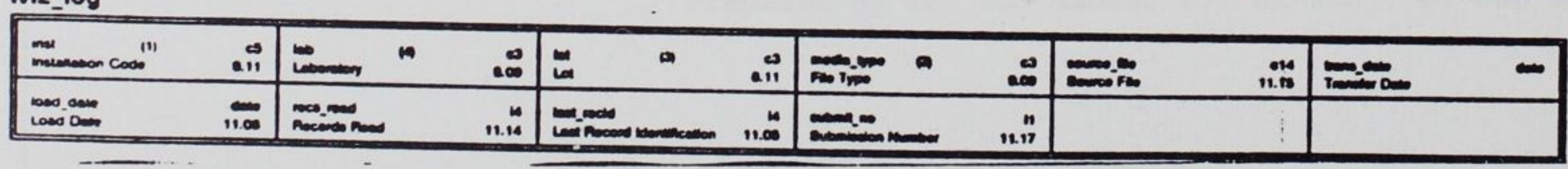

LEGEND

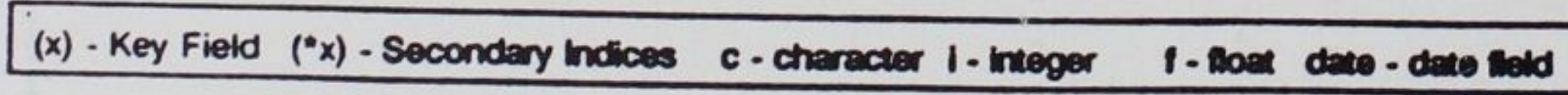

1 Aprill 1991 
ELEMENT IS USED IN THE FOLLOWING IR RECORDS AND DATA BASE TABLES

\begin{tabular}{|c|c|c|c|c|c|c|c|}
\hline 'an' & conson & m. & 1002 & Con & וסומה & I & $\infty \mathrm{c}$ \\
\hline נוn & & 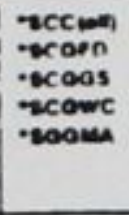 & & $\begin{array}{l}\because: \\
\because ; \\
\text { is }\end{array}$ & $=$ & & $\equiv$ \\
\hline
\end{tabular}

ELEMENT SIZE AND CHARACTERISTICS:

defined.

S alphanumeric characters, left justified. Only the first nowo characten are presently

ELEMENT DESCRIPTION:

Code identifving the name of the installacion from which date is being collected. aCCEPTABle CaITERIA:

- Required in File Name and in records indicated above

ACCEPTABLE ENTRIES

(Listed alphabecically by installacion Code; vee abo 11.04 tratallation Name)

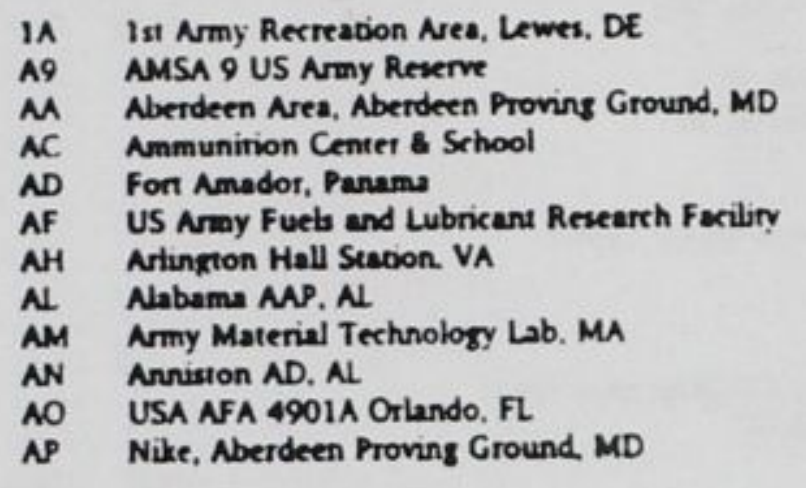

14ard 1901

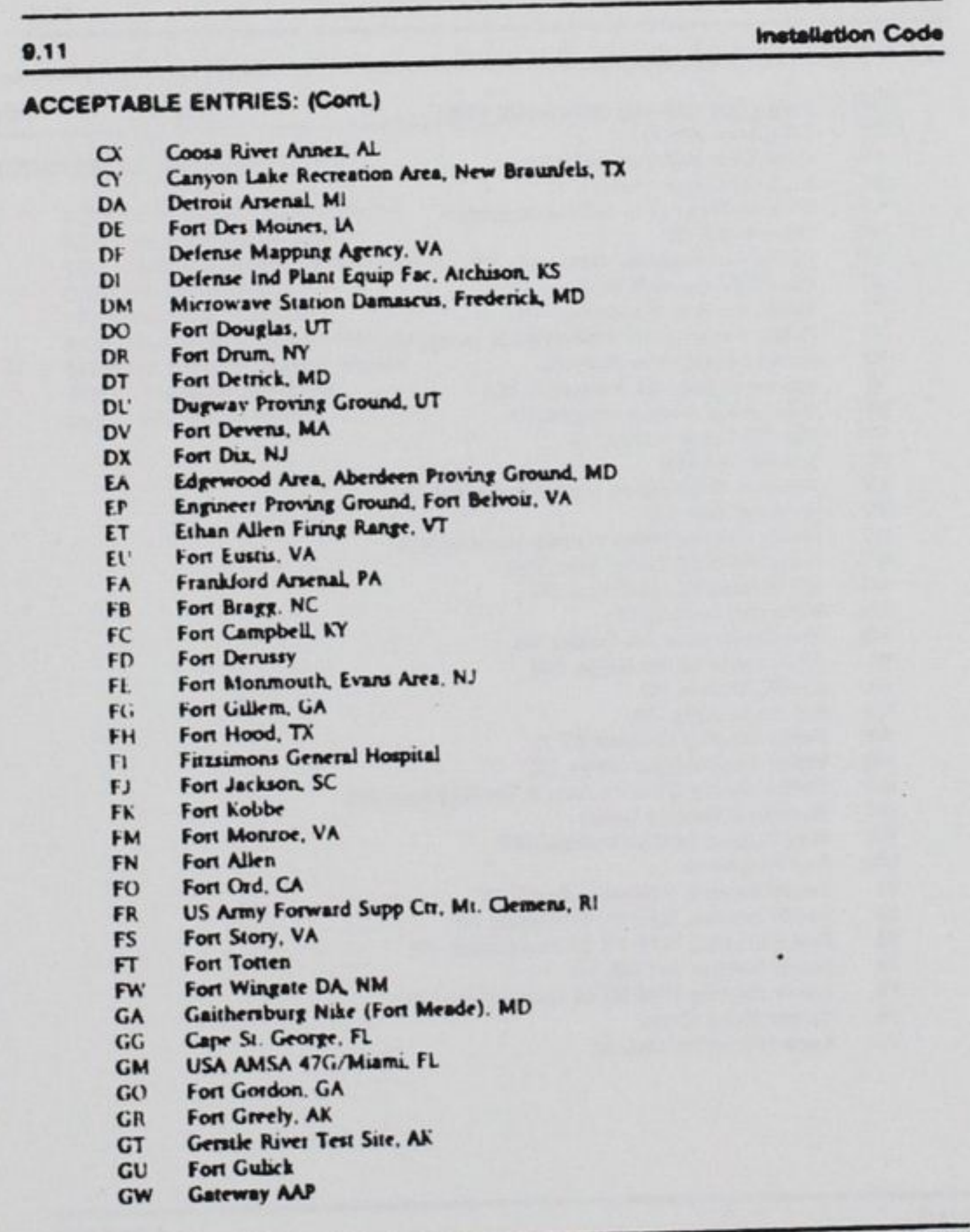

1A001 1001
ACCEPTABLE ENTRIES: (Cont.)

Numament Aunutions Chemacal I ammand

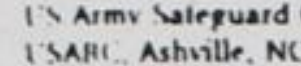

Aadye+1 MP. WI NC MI

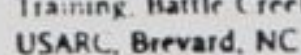

Alue Gisss Nea, Lexingrion Blue Grass DA $\mathrm{Kr}$

Fon Renjamin Harnson, iN

Fon Baket
For Bliss.

intenance

Bensett Army Nocional Guerd Fecility, CO

Blosiom Point Ordname Worta MD

Fed Repromal Center Broctell Brothell, wA

For Burhanon, PR

Fon Betroi, VA

Bayonne Militery Oreean Terminal, N.

Family Housing Pleinnilte. CT

Family Housing Faurfield, CT

Famuly Housing Windeor, CT

Family Housine Middletown. CT

Family Housing Orange, CT

Fomily Housing Portend, CT

Carliste Barrack

Corpus Christi ND. TX

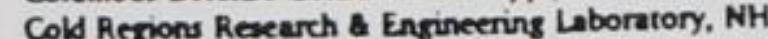

Fort Chatiee

Cherketion Amay DA

Family Housing PITT 42 Ehirabeth. PA

Cemeron Station, VA

Fort Canon $\infty$

Comp parts Livermere a

Grane Naval Weapons Support Center. IN

Comp Sums

Fort Clayton
Grane Arny Ammunition Activing

0.112

1 apent 1001

Installation Code

0.11

ACCEPTABLE ENTRIES: (Comt.

HI Family Housing Hull MA 36

H3 Hunter tis Middle Marker, Surannaht, GA

UA Hawihorne MP. MV

B Fon Holabird. MD

MD Harrv Uramonu Laboratories

HE Hek Dod Sonts Row Paro Ronles C

Hil tort A P. Hill VA

ith Fon Huniet Legett. CA

IM For Hamilion

HN For Hankoxk

HO Hobion MP.

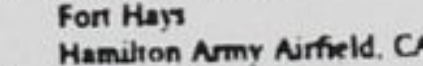

Fon Huarhuca. AZ

Hones MP. PA

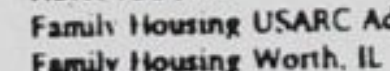

Family Housing Worth. It

Lown MP. IA

Fon Indiantiown Gep. PA

Indiana M

Imun Support Det Arnez. Mekeespon. PA

Family Housing NIKE Phila $41 / 43$ Clemnen, NJ

Family Howsing NIKE MY

Fanily Howna NIKE MT

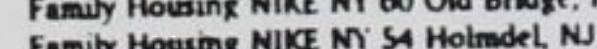

Jefterion Berracks LDF

Jefterion Proving Ground, IN

Jobiet MP. II.

Kaneas MP. KS

Kings bay Mbiary Oreen Terminal

(ing $30, \mathrm{MO}$

Kopaleme Military Revervation. III

For Knor. KY

Litert Dam. Baltimuie. MID

Late Ciry MP. MO

Fort Leonerd Wood. MU

For Lee, VA 
011

ACCEPTABLE ENTRIES (Cont)

inotallation Code

1 Benghnen AN

Lamas Armi Mowitio

Lake lawen Neveth Gulth Sutr 1

Lume oln Supynut fardits Smuthineld. RI

Lone Star MP. TM

USARC LI

USARC. Lumberion. $N C$
Fon Leavenworth. KS

Forl Lewis. WA

Lexington Ares, Lexington-Blue Grass DA, KY

Family Housing Topatreld, MA

Family Housing Randolph. MA

Family llosing bevelly, MA

Fomily Housing Buringeld, MA

Family Housing Bedford, MA

Family Housing Swansea, MA

Famuly Housing NIKE Wesh Balt 35 Croom MD

Family Housing SLAsc Wherry. MO

For Mousing Mancheter CT 25

For Meade, MD

Memphis Delense Depor

Michigan MAP

Milan ANP. TN

MCNester MP. OK

Fon Monmourth Main Post, $N$

Fort McNaur

Family Housing Milford CT 17

Fort McPhernon, GA

Mississippi Aup, MS
Fort MacArthut

Fort Missoula, Fort Massoula. MT

Matenel Development and Readines:

Fort McCoy, Wi

Mort McCoy, W

Mavajo AD, NZ

Fandily Housing New Britain CT S7

140011001

Q.11-

$9.11 \quad$ motellotion Code

ACCEPTABLE ENTRIES: (Comt)

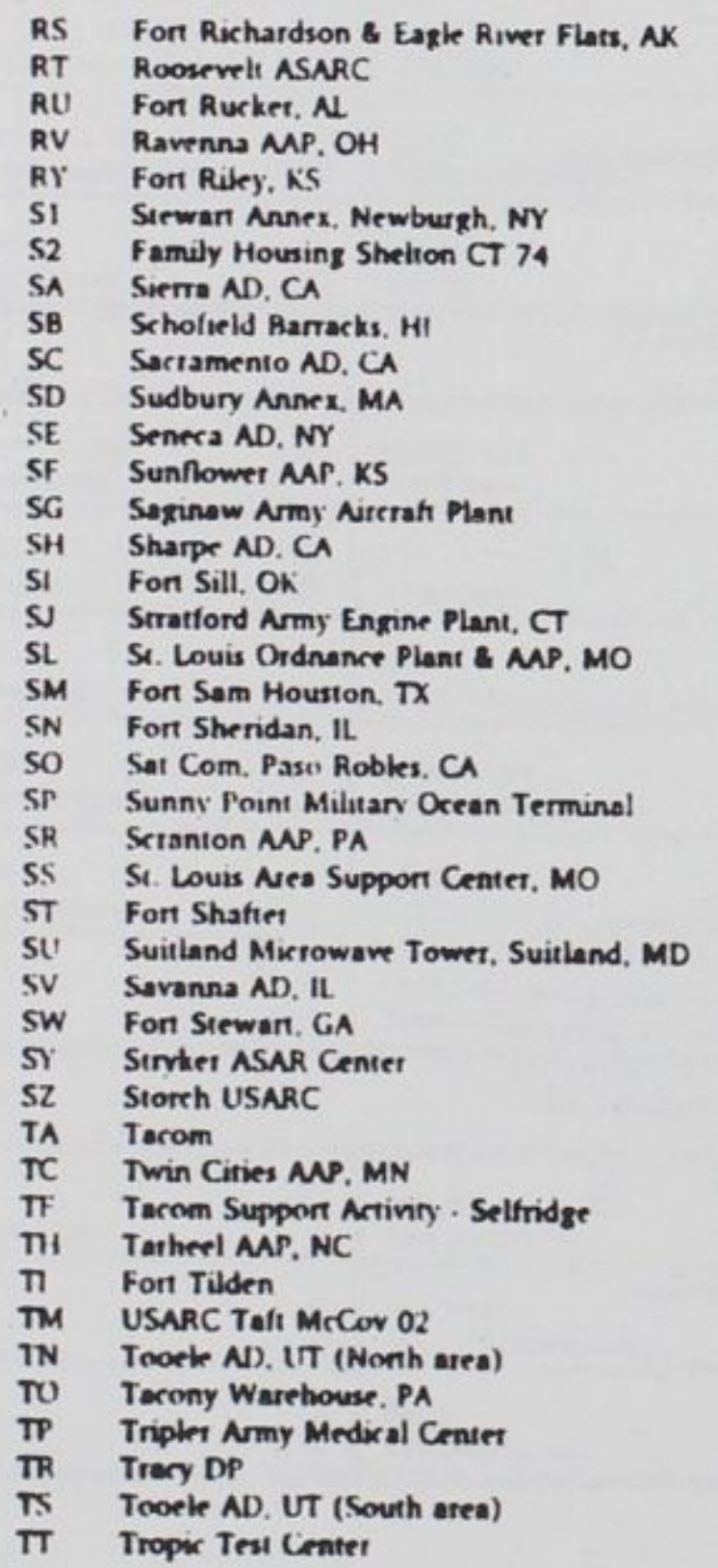

1 A001 1001 ingtellation Code

ACCEPTABLE ENTRIES: (Cont)

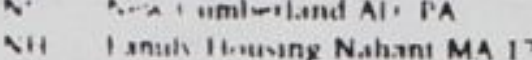

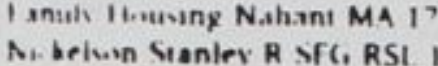

Aatub Hilt, MA

New Citreans Midarar Ocean Terminal Lu

CFI N' Britan USAR Centet

Chabdale Support Center

(S)den velense Depor, U

Cakdate Army Base

Family Housing PTT 03 Doneyville. PA

Family Housing PTT O2 Herminue, PA

Family Housing Irwin SPT DET ANX PA

famuly Housing PITT 25 Monroenlle PA

famuly Housing PITT 52 Finjeyville. PA

Famuly Houssing Coraopolis 71 (PI 71L). PA
Family Housing Coroopolis 72 (PI 7IC). PA

Famuly Housing Coraopolis 72 (PI $71 \mathrm{C}$ ). PA

Rine Bluft Arremel, AR

Phorphate Developence

Philadelphis Defense Penonnel Supporr Center, PA

Pratinny Arsenal. N

For Pickett, VA

Fon Polk LA

Pontier Siomenterey. CA

Presidio of $\operatorname{San}$ Francisco, $C$

Puebto DA CO

Phoenix Military Reservation MD

Quarry Height

Family Housaing Davisville, RI

Fomily Hovaing N Smithfield, R

Redstone Areenal, $A$

Richmond Defense General Supply Center

Redilord MP, VA

Fon Richtio

Rock lisend Arenel, II

(dota after 1964)

Rocky mountain Arenal, $c 0$

2.114

14001

\begin{tabular}{ll}
\hline motelnotion Codo & 9.11 \\
\hline
\end{tabular}

ACCEPTABLE ENTRIES: (COML)

TW Twin Cities MP. MN (data belore 1986)

Tobyhanna AD, PA

US

US Army Houstion Armed Forces Center

Umarillo AD. OR

US Miltary Arademy, West Point, NY

Jasac Tampa 4015 W. $T L$

Family Howing NINE Nortol VA P. Heng. VA

Family Housing Manoum $V A$

Vancouver Barreckse. Vencouver, WA

Vaber Forese General Houpienl PA

Vint Hill Farms Station, VA

Van Nuyn Maintenance Shop, Los Angeles, CA

Fomity Housing wA

Fandy Hovering Youngs Late, WA

Family Housing Sun Praine, Wr

Waterubet Anenal, NY

Wodre Soge Research Facdin. VA

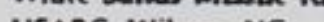

For Weinwrighe AN

Fanily Hovaing Westpon CT 73

Mant \& Trining Avea, MO

Fen

Family Houning NIIE NY 25 Rocky Point. NY

Family Houning Dry Hill NY

Yacuma furing $c$ 
ELEMENT IS USED IN THE FOLLOWING IR RECORD AND DATA BASE TABLES

ACCEPTABLE ENTRIES (CONT)

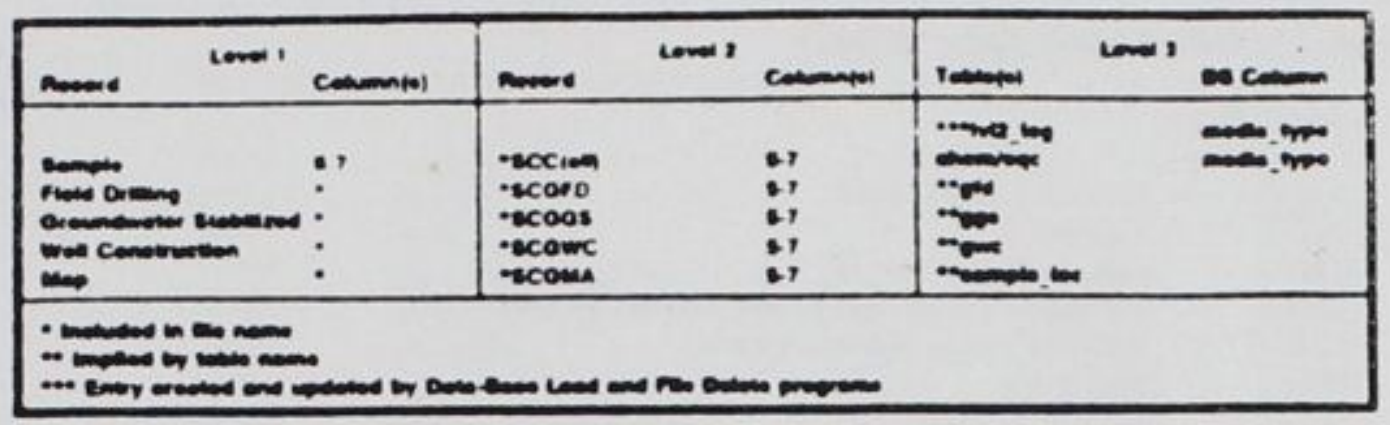

ELEMENT SIZE AND CMARACTERISTICS:

3 upper-case alphabencal characters, full field

ELEMENT DESCAIPTION:

Code identifying the rype of dars.

ACCEPTABLE CRITERIA:

- Required for all renords (explicitly or implicitly es indicated above)

ACCEPTABLE ENTRIES

(- Fllo Type not currently in wee)

CAP Chemical Anelyzris Paint

CAR Chemical Nis
CAT Chemical Animal Tissue

CAT. Chemical Animal Tissue

CBE. Chemical Benthic

CBI Chemical Building - Interior

CBS Chemical Building

CBX Chemical Buildieg - Exterio

CCM. Chemical Composite Semples

CCO. Chemical Concrete

CDM Chemical Drums

CDR. Chemical Drain

CGW Chemical Grownd Water

31 anower 1000

IM. ( hemical Influtation, Waies

(MI)" (Mremoal Merthuds IL

CP Chemical Process Contro

CPW. Chemical Powder well

CQC Chemasal Oualivi Control

COR. Chemical OC Prman Standard

CSD Chemaral SLandards Developmen

CSE Chemical Sediment

CSO Chemical Surety Materis

CSO Chemical Soil

CSS Chemaical Staintess Sier

CSu Chemical Sump

CSW Chemical Surtere Water

CTF Chemical Transforme

CT.' Chemical Tite

cWD. Chenical Vent

DTT Deron Treatability Matrix

EGO- Exological Generol Obsemations

EMO•-Ecological Mecroonverebrate Observations

EOC. Ecological Orgenism Code

ESP. ENological Semple Preparation Fil

EWV. Erological Herbage Vesetarion

GAO Geofecthnical Aquider Aratyris

GEL. Geotechnical Elevation File

GFD Geotechnical Field Drilling

GGS Georecthnical Ground Water Stabihized

GMA Georechnical Map

GMD" Geotectinical Methods Description

GMS" Geotechnical Methods Summary

GOR. Geotechnical Origin

GPA Georechnical Physical Aralysis

PAT" Gorechnical WeV Construction

MTT Rolloson nostewent Treacment

RMT Radiological Arimal Tave

RBX Rediotogical Buidans intenior

RGW Rediologicel Ground Went

RPT• Rediological Plant Trere

0.09

Fuo Typo

ACCEPTABLE ENTRIES: (CONT.)

ROC. Radiological Qualiry Control

RSE Radiological Sediment

RSI. Radrological Survey

RSR Radiological Sonver

RSW Radiological Surfece Woter

RWT: Radiologncal Surfece Wiping

TOPS Trearment operamions 
ELEMENT IS USED IN THE FOLLOWING IR RECORDS AND DATA BASE TABLES

\begin{tabular}{|c|c|c|c|c|c|c|c|}
\hline Aneres. & cones & Conmontol & Marere & 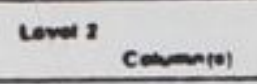 & וסוד & cons & $\infty$ cen- \\
\hline 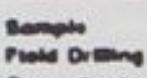 & & $\because "$ & scc & :": & - & & $-m$ \\
\hline$=0$ & & 翟 & 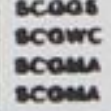 & 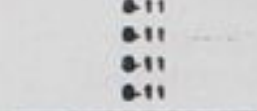 & $=$ & & $=\infty$ \\
\hline
\end{tabular}

ELEMENT SIZE AND CHARACTERISTICS

4 uppei case alphabetic characters, teft jusrified

ELEMENT DESCRIPTION:

A standardized code representing a rype of landmark, fearure, or construction.

ACCEPTABLE CRITERIA:

- Required on all rerords

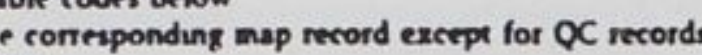

must be blank

ACCEPTABLE ENTRIES:

(The ette typees ere lleted by deta type for eese of wee.)

Chemical or Rediologicel Data:

Chomicel Analyois Poimt (CAP)

BLDG building

FBLK field blank

SURF surfeces in seneral

TRIP trip blank
Site Type

ACCEPtable EntRies (Cont.)

Themical or Rediological Data: (Cont)

Alt (CAR):

ARM() aut monitoning sation

BLDG buildins

CMPG Composite grab somple

composite sample colen multipte loration

chemisal aludge disposal renther

OLSP old lagoon aludge pik

RNSW rinsewater

TRIP Trip blant

TUNL tunnel

UNKG unknown grab somple

Animal Treaus (CAT or PAT):

BIOL biolonical sample

CMPII composite sample taken from multipte locations

FBEK FiK field blan

WAKE lake

RNNW pond

RVER NRSEW

STRM Trer

SURF surfoces in general

TRuP trip blank

Buliding intorlor (CBI or ABI)

ASPH esphats

BLDC building

CASE casemale

CMPH composite sample taken from multiple loc ation

CONC concrete

FBY Reiling bie
9.17

ACCEPTABle ENTRIES: (Cont)

Chomicel or Radlotogicel Dato: (ComL)

Bullding Interior (CBL or RBI): (COOL)

FELD freld

GSDA mease or sludge disposal area

PLAS

RNSW

TRIP imp blank

WINS nivil noor til

WIPE wipe
WLBD wall board

Whon wood

WTL wall tile

Bullding Survoy (CBS):

BATT batrery

BLDC building
CASE cesernate

CMPH romposite sample token from multiple beration

FBLK FW field blank

RNSW nipsewore

TRIPL tip blant

Chomical Blou (CBT):

BIOL biological semple
FBLK field blank

RNSW rinsewater

TRIP trip blank
Sho Typt

\section{She Type}

ACCEPTABLE ENTRIES: (Com.)

Chemicel of Radologicel Dore: (Comt)

Bullding Extertor (CBX or RBX)

ASPH osphalt

BLDC buildin

CASE casemose

ind

cencen

CONC EONCrete

GSDA srease or atudore dipposel ares

RNSW rinsewnter

ROOF roofing moterial

SHGL shingle

TRIP trip blank

WIPE mpe

Drum (COM):

DRUM drum

FBLX field blan

RNSW rinsewar

TRIP trip blank

UFS unknown fobriceted oubrtaone

Grounownter (CaW oe RaWn:

CMPH composine sample taken from multiple bocation

Bux

FLPL
LYSM Aloodplain
hyimetet

OTFL ourtall

RNSW rinsewater
SPRG

STTK eptix tan 


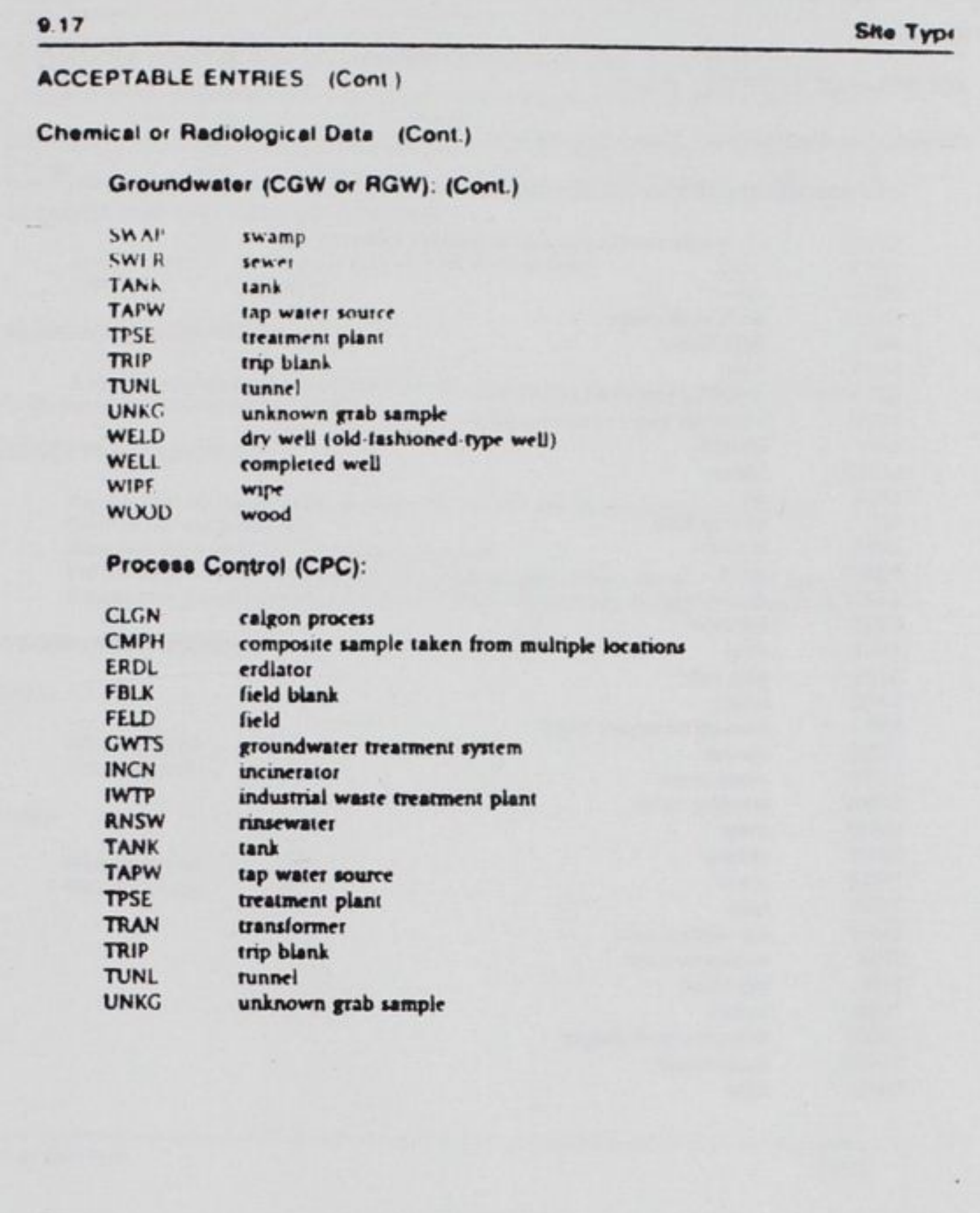

140011 1001

9.17

ACCEPTABle ENTRIES: (Com)

Chemicel or Badiological Data: (Com)

Sodiment (CSE or ESE): (Cons)

\begin{tabular}{|c|c|}
\hline $\begin{array}{l}\text { WFL } \\
\text { LAGO }\end{array}$ & $\begin{array}{l}\text { landfill } \\
\text { lagoon }\end{array}$ \\
\hline & lake \\
\hline $\begin{array}{l}\text { MAHO } \\
\text { MT }\end{array}$ & manhote \\
\hline $\begin{array}{l}\text { MT } \\
\text { OTFL }\end{array}$ & $\begin{array}{l}\text { marshy area } \\
\text { outfall }\end{array}$ \\
\hline PLUG & shovel remple \\
\hline POND & pond \\
\hline PRSW & processs sewer \\
\hline $\begin{array}{l}\text { RNSW } \\
\text { RSVR }\end{array}$ & rinse water \\
\hline & resenvit \\
\hline $\begin{array}{l}\text { RVER } \\
\text { SKHL }\end{array}$ & niver \\
\hline $\begin{array}{l}\text { SKHL } \\
\text { SPRG }\end{array}$ & sink hole \\
\hline $\begin{array}{l}\text { SPRG } \\
\text { STP }\end{array}$ & $\begin{array}{l}\text { spnne } \\
\text { senitan trearmens plans }\end{array}$ \\
\hline $\begin{array}{l}\operatorname{STP}_{\text {STRMI }} \\
\text { STR }\end{array}$ & $\begin{array}{l}\text { sonitarv treatment plant } \\
\text { stream }\end{array}$ \\
\hline STSW & $\begin{array}{l}\text { stream } \\
\text { storm sewet }\end{array}$ \\
\hline STwA & randing water \\
\hline SL'Mr & sump \\
\hline SURF & surlaces in general \\
\hline SWAP & swamp \\
\hline SWER & sewer \\
\hline TANK & tank \\
\hline TPSE & treatment plant \\
\hline TRIP & $\begin{array}{l}\text { irip hlenk } \\
\text { then }\end{array}$ \\
\hline TUNL & runnel \\
\hline UNKG & unknown grab sample \\
\hline WASS & colid waste \\
\hline WELD & dn well (old-le \\
\hline WIPE. & \\
\hline wOOD & wood \\
\hline
\end{tabular}

14001001
She Type

ACCEPTABLE ENTRIES (CON!)

Chomical or Rediological Dete (Cont)

Plent Theove (CPT or RPT

AI: II brotorical sompie

MPH composite ample taken from multiple torenons

FBik hield blank

vaste trearment plant

PLUC shovel sample

POND pond

SURF surfeces in genera

TUNL mp blank

WOOD

Standerde Davetopment (CSD)

EVAL evaluation

FBLK field blonk

TEST tert

TRIP trip blant

TUNL Tunnel

UNKG unknown grab sample

Sedimont (CSE O RSE):

BASN besin

BORE bore hok

CMPH cistern

CREK Treek

DTCH drum or drainage

FBLK fireld blank

FELD field

FLPL Roodplain

grease or sludge disposel ares

induscrial waste trestienet plent

0.174

12001001

Sno Typo

.17

ACCEPTABle ENTRIES: (Comt)

Chemicel of Racllological Date: (ComL)

Suroty Metorlal (CSM):

FBUK field blank

TRIP trip blank

UNKG unknown grab rample

SOll (CSO or RSO):

AREA ares of lend

OLSN basin

BORE bore hol

BUGR buming groun

conilerous deriduoes moodian

composiate menpte taken fron multiple locations

CREK

CSDT chemicel aludare dirponal orencher

DEMO demotiona area

DTCH ditch or drainage.

FBLK field blent

FEL

FLPL. Noodploin

GSDA greeme or aludoge dieposed area

WFL lendfII

MAGO lagoon

OLSP old laproon etudge pite

OTFL ourtall

Prit piefree apede

QNSW rinse water

SKHL sink nole

STSW Storm rewe

SURF sump sures in genert

SWNP surfores 


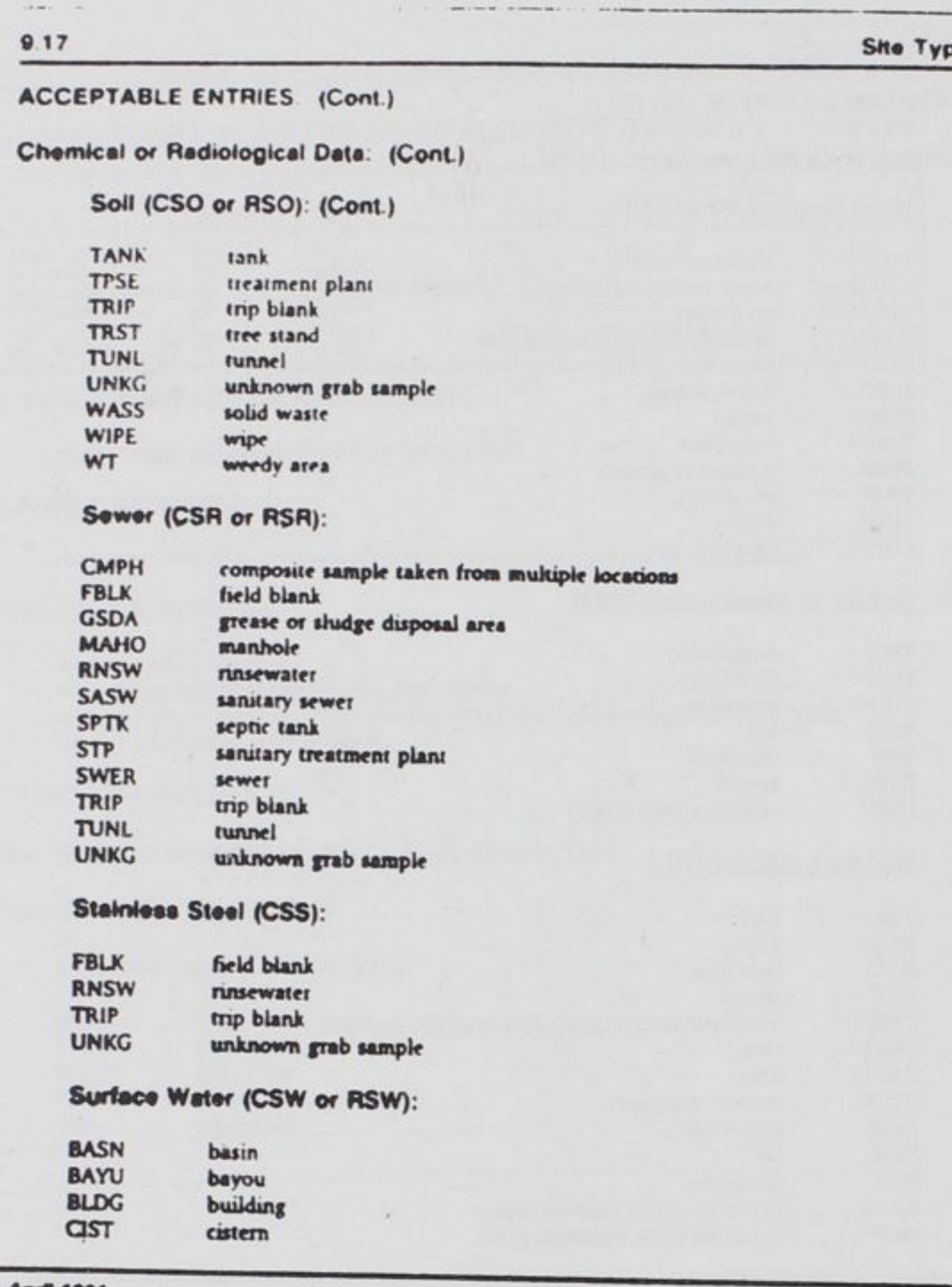

140.t 1901

2.170

SHe Type

ACCeptable entries (Cont)

Chomical or Radiological Data: (Cont.)

Surface Wator (CSW or RSW): (Cont).)

CMPH somposite sample taken from multipte bocenons

CREK Cleek

DTCH ditch or drainere

FBLK field blank

FELD fielo

GSDA grease of sludge disponal area

industrial waste treatment ph

UGiO landfil

UKE lake

MT manhy area

OTFI outiall

RNSW rinse wore

RSVR reservo

RVER R rivet hol

STP anitary reacment plant

STRM stream

STSW norm newer

STWA randing wo

SUMP Bump

SWER TWE

TAPW tap wreer source

TPSE treatment plant

TRIP trip bleak

TUNL Tunnel

unknown grab rample

WIPE bquid wase

9.17

$2.17 \cdot 10$

1001

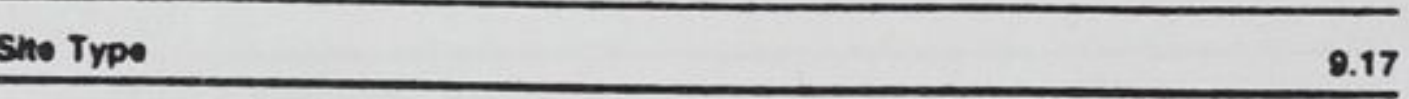

Flold Driling (GED):

BORE bONe

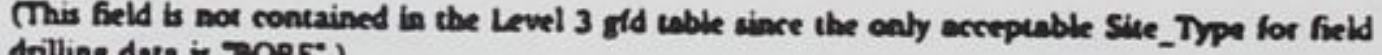
Gilling dots is BORE:)

Woll Conetruction (aWC):

WELl completed well
IYSM

Mep Dete (ama):

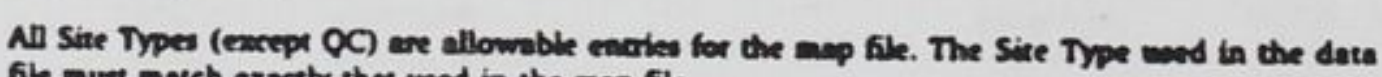

Other Data:

Unoxploded Ordinence/Motal Obloct (USS):

UNKC unknown grob remple

\section{7}

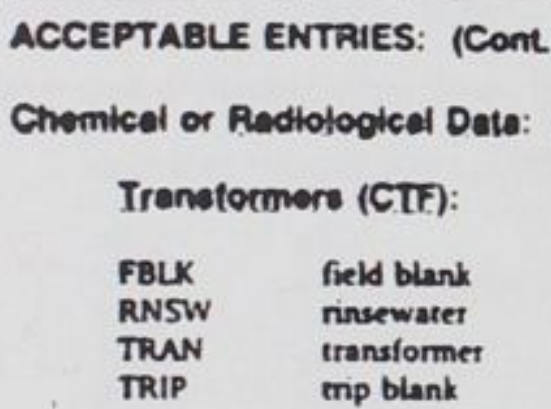


8.05

Depth (Chemical)

ELEMENT IS USED IS THE FOULOWINO IA RECORDS ANO DATA BASE TABLES

\begin{tabular}{|c|c|c|c|c|c|}
\hline r. & ron' ' & זי & " l' & $\mathrm{ram}$ & 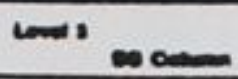 \\
\hline$\infty$ & , & $\mathrm{cos}$ & $=0$ & همam & $\infty$ \\
\hline
\end{tabular}

ELEMENT SIZE AND CMARACTERISTICS:

Levels 1 and 2: Derimal (5 digits plus decimal point)
Level 3:

ELEMENT DESCRIPTION:

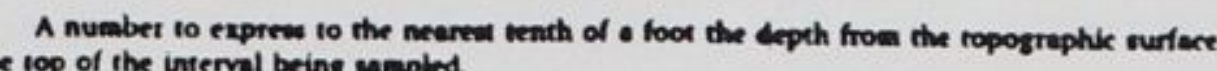

aCCEPTABle Carteaia

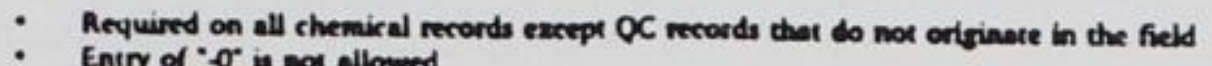

Entry of - $\sigma$ is mor allowird

Musi be a derianal to the neareat rench of o look

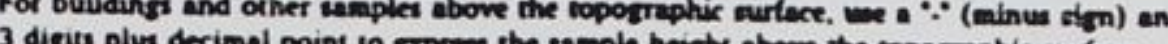
ACCEPTABLE ENTRIES

Depth:

$\begin{array}{ll}\text { Minimum value } & 0.0 \\ \text { Maximum value } & 0.099 .9\end{array}$

Height:

Minimuma value
Maximuma value
8.19

Sample Date

ELEMENT IS USED IN THE FOULOWINO IR RECOROS AND DATA BASE TABLES

\begin{tabular}{|c|c|c|c|c|c|}
\hline Nos & 1 & ner & lond & $100+1$ & (nest) \\
\hline 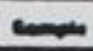 & $\infty n$ & cans & $m$ & דוم- & تصه \\
\hline
\end{tabular}

ELEMENT SIZE AND CHARACTERISTICS:

Level 1: Becharacters - format (MMDDOM

Level 2: Julian date (TrDDO

ELEMENT DESCAIPTION

Dote on which the sample was taken

ACCEPTABLF CRITERIA:

Valid date

ACCEPTABLE ENTRAES:

Minimum: $\quad 2-1 \tan 75$

Maximum: <- Sample Pieparation Date

¿- Aralyents Date 
8.03 Analysis Type

ELEMENT IS USED IN THE FOLLOWING IR RECORDS AND DA. A BASE TABLES

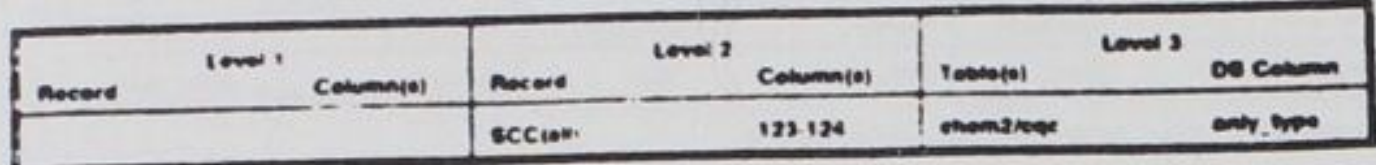
ELEMENT SIZE AND CHARACTERISTICS:

$$
2 \text { characters. full field }
$$

ELEMENT DESCAIPTION:

$$
\text { A cade that represents the certification Invil for the analyos It in goneroted during }
$$

Centification

(1) mecthed.)

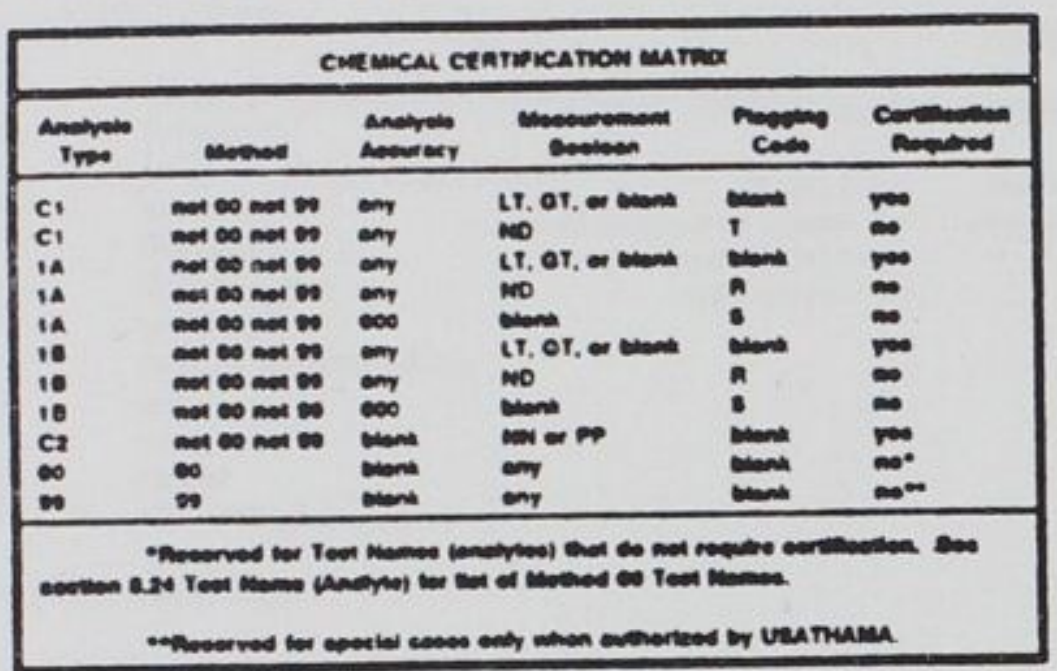

ACCEPTABLE CRITERIA:

- Required lor all level 2 and 3 chenical rererde
aCceptable ENTRIES:

SCC Rerords:

C. Class 1 (quantitative)

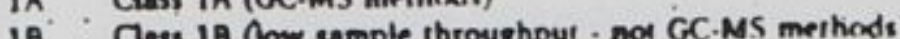

C2 Class 2 (anolinetive)

00 Special Teat Names that do not require certification

99 Sperial cases only when authorized by USATHAMA

SAC Rscords (obrokete)

ON Quantitative methodology,

SO Semiquentitative methodology

\begin{tabular}{|c|c|c|c|c|c|c|}
\hline 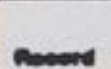 & 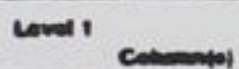 & mor & ${ }^{200} 2$ & 1 & cond 3 & 0 \\
\hline דهenמ & $\infty$ & $\underset{c \infty}{\infty x}$ & $\lim _{i=1}$ & $=$ & & $=-\infty$ \\
\hline
\end{tabular}

ELEMENT IS USED IN THE FOLLOWNG IR RECOROS AND DATA EASE TARLLS:

ELEMENT SIZE AND CHARACTERISTICS

ACCEPTABLE CATTERU: (CONT.)

overemons:

blank

menes

blank

nomes in

Ulenk

Badtotosicel Deta:

Decimel number benwen ".001' and 999." (zero and blank not allowed) ACCEPTABLE ENTAMES:

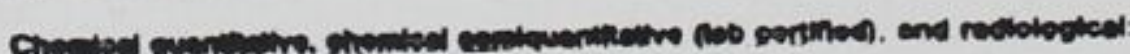

Mintrimer: $\quad \begin{aligned} & .001 \\ & \text { Marimum: }\end{aligned}$

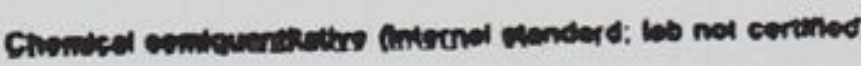

$\infty$

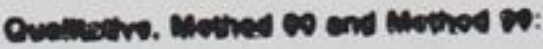

Blank

Must be e decimal number between ".001" and 999 "

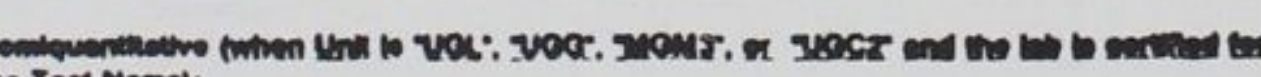
Ten Neano:

Must be a decimal number berweren " $.001^{\circ}$ and $999{ }^{\circ}$

Zero and blank are not allowed

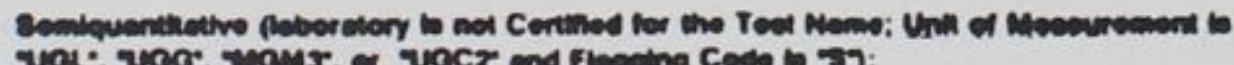

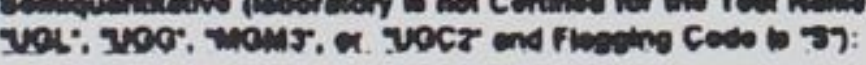

noo: 


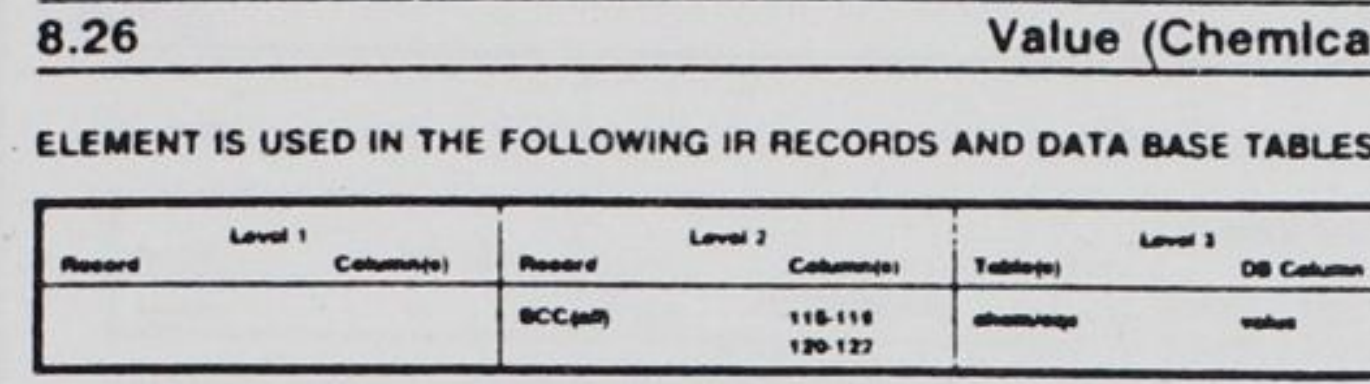

ELEMENT SIZE AND CHARACTERISTICS:

Level 2: Mantisss - 4 digits plus decimal point, nght justified

Level 3: Fipart - 6 digits plus decimal pount

ELEMENT DESCRIPTION:

Value of the analysis dependent on Test Name and Unit of Measurement. The Value is computed by one of the following rwo equarions:

EQUATION

(1)

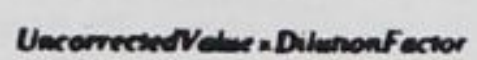

\section{DATA TIRE CONDTHON}

Non-COC Measurement

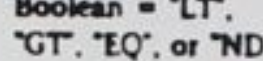

$\operatorname{cQC} \quad Q C$ Tet $=5, M$ of ' $\mathrm{N}$ ' and the Boolean a IT. TT. Boolean a "LT. CT
EQ. ND., or blank)

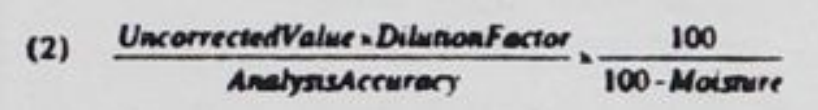
Non-COC Measurement $\operatorname{COC} \quad$ OC Test $=R^{*} \cdot T$

\section{ACCEPTABLE CRITERIA}

- Required for all chemical ierords.

See wertion 8.25. Uncorieried Value, Ior maximum number of significant figures

\section{ELEMENT IS USED IN THE FOLOWING IR RECORDS AND DATA BASE TABLES}

\begin{tabular}{|c|c|c|c|c|c|}
\hline סמשח & 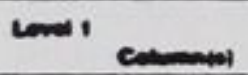 & ניחנ & ו ו & 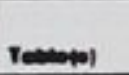 & 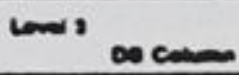 \\
\hline זسمس & $\infty$ & 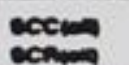 & הen & $=$ & $=-$ \\
\hline
\end{tabular}

ELEMENT SIZE AND CHARACTERISTICS:

2 upper ease alphaberical charactern, full ficld (blank allowed)

\section{ELEMENT DESCRIPTION:}

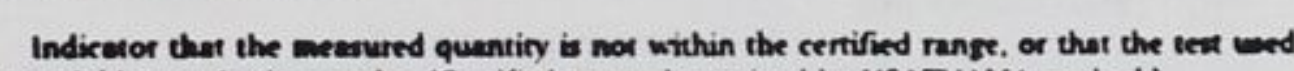
does not yeld quancicative resulta (Centifed range determined by USATHWMA method.) ACCEPTABLE CRITERIA.

- Must match an arreptable code below for the level of cerrification pertinent to the rerord

$$
\text { in question. }
$$

The value must be at the maximusa cerrified concentration when "CT is uned for

quantuative or semiquantasine mords

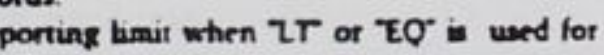

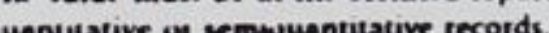

ACCEPTABLE ENTRIES

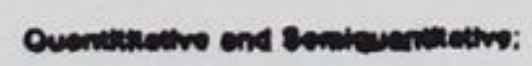

$$
\begin{aligned}
& \text { EO equal to the Centified Reporting Limi } \\
& \text { LT less then Certified Reporting Lumit } \\
& \begin{array}{l}
\text { GT greater than maximum certified concentiotion } \\
\text { blank }
\end{array}
\end{aligned}
$$

\begin{tabular}{ll} 
Value (Chomical) & 8.26 \\
\hline
\end{tabular}

ACCEPTABLE ENTRIES:

Lovol 2

Marwees:

Minumum: $\quad 1.000$

croones:

Minumum: 6

Lonets:

Minimum: $\quad .000001$

Meceuremem Booloen $\quad 2.12$

ACCEPTABLE ENTRAES: (CONT.)

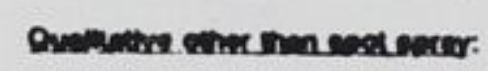

EQ equal to detertion livait
IT

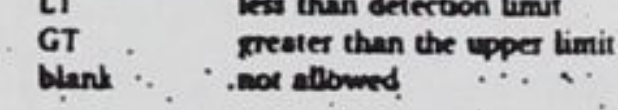

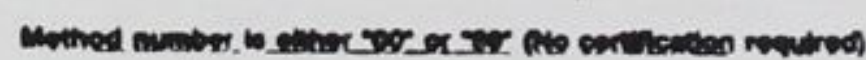

LT VTe than deterion limit

Whant for all ocher cosper tim

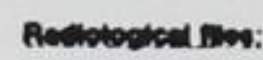

BB below background

LT less then detection limit

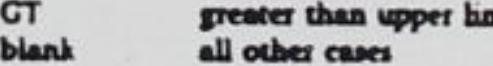

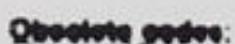

OD positve odor lest
Q1
qualitative - insermel standerd 


\subsection{9 \\ Unit of Measurement}

ELEMENT IS USED IN THE FOLLOWING IR AECORDS AND DATA BASE TABLES

\begin{tabular}{|c|c|c|c|c|c|}
\hline nome & $c$ & $\rightarrow$ & |cos & 1 & 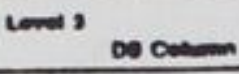 \\
\hline$\prod_{n=0}^{\infty}$ & (3) & 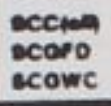 & (⿻) & $=$ & $==$ \\
\hline
\end{tabular}

ELEMENT SIZE AND CHARACTERISTICS:

4 alphanumeric character, keth justified (no embedded blanks)

ELEMENT DESCRIPTION:

$$
\text { Code representing the Unit of Measurement of the Value. }
$$

\begin{tabular}{|c|c|c|}
\hline Promx Coso & Promx & Pomm of 1 \\
\hline A & anto & .18 \\
\hline $\begin{array}{l}F \\
p\end{array}$ & Iemto & .15 \\
\hline $\begin{array}{l}\mathrm{P} \\
\mathrm{N}\end{array}$ & piro & -12 \\
\hline${ }_{U}^{N}$ & nano & 9 \\
\hline $\begin{array}{l}U \\
M\end{array}$ & тікто & 6 \\
\hline$M$ & milli & .3 \\
\hline$k$ & tulo & +3 \\
\hline ME & mege & +6 \\
\hline G & sg8 & +9 \\
\hline T & iere & +12 \\
\hline PT & peta & +15 \\
\hline & exto & +18 \\
\hline
\end{tabular}

\section{ACCEPTABLE CRITERIA}

Required on all records that contain an uncorterted mantisas value

Blank fot records where the Measurement Bookean is "NN" of TP

Blank for records where Test Name a "PH

9.19

Unh of meecuremem

ACCEPTABLE CATERLA: (CONT.)

Rediolooted Dete:

unir oeschionow

CM2P pirocunes/2quare centimetes

CPM counts/minute

CTS counts

ns/minute/squere meter

(a)

DPMA disintegrations/minute/100 square col

FCL femtocunies/liter

FCG temtograms/gram

GL Iemtograma/liner

MCM millirem hour

AICMII. muerexunes/miltimeter

MGGA milligram gram-ash

MRAD milltrads

MRII millirem hour

MRM millioentrens/munute

NCL nancrunes. iter

NCNG nanoxuries nanogram
NGiGi nanograms' ram dry

NGL nanograms'liter

PCC prooruries

CCG picocuries gram

CGA pikoruries gram-ash

CGD picorunes/gram-dry

CCLW picorunes/gram

CCM2 pirorunieviquare meter

PCMIL pirorunes/millibiter

UC mikrorunes

UCGA microxunies/gram ash

ICCDD mitroruites Riam din

CCGW mikricures gram wo

UC. motckuties liter

KCML mictiruires/milhitie
Unn of Meesurcment

ACCEPTABLE ENTRIES

Chomical Deta:

$\begin{array}{ll}\text { UNTT MEDAA } & \text { DESCRIPTION } \\ \text { UGL Liquids, drums } & \text { mirrograme liter }\end{array}$

UGL bquids, drums micrograme titer
UGG solids. drums micrograma/gram

UGM3 gases micrograma/cubic meter

UGC2 surfaces micrograma/ aquare centimeter

Soectal Coser (Mothed 00):

UNT DESCRIPTION TEST MAME(S)

blank unitless

CPML CounV $100 \mathrm{ml}$ TOTCOL

COLOR

NGBEST (and other asbestos Test Names)

NTU nephalometrix rerbidity unit

ASBEST (and ather abbestos Test Namer)

TDN caste dilution index number TASTE

UMHC micromho/em-ronductiviry COND

Queliny Control Data:

unT Descrention

BLANK PH

MOUP mole percent

PCTP percent phosphorus

PPB parts/billion

PPK parts/thousand

PPM . Parta/miltion

PM parts allition

0.102

1 14001 1901

Unth of Mecevurement

9.19

ACCEPTADLE CARTERUA. (CONT.)

\section{Onetectioled Dats:}

Eledd Daing Datio:

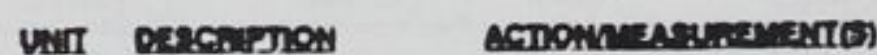

BL hamer blom Hasto

TT Det DERK DRTOT, GROWT, RECVR, NFUSL

MIN minutet BNLT, RECVT. TME (depending on Method)

PSI poundwaquere inch TMEE (depending on Method)

SEC N ADVAU. BFILL BSTAT, CAVEI COLOR, CONSS. DRTVE NDVAU. BFLLL BSTAT. CAVEL COLOR, CONSS, DRIVE. USCS

Woll Cenerruction Dore:

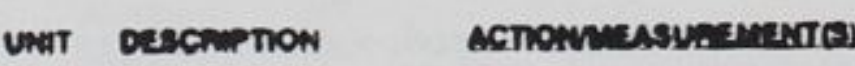

FT leet CASE, CASED, CASES, CSENL, DPTOT, LYSDP, RECVR.

then

$\begin{array}{ll}\text { MIN mumutes } & \text { BNLT, RECVT } \\ \text { blonk } & \text { BFLL, BSENL, GFILT, GROUT, SCREN, SURF, TOPO }\end{array}$ 
ELEMENT IS USED IN THE FOLLOWING IR RECOROS ANO DATA BASE TABLES

\begin{tabular}{|c|c|c|c|c|c|}
\hline nesere & 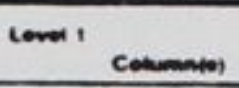 & بתس & $\operatorname{lom}^{2} \mathrm{C}$ & $1000+01$ & Lons \\
\hline - & 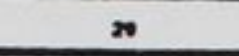 & sections & 100 & $-\infty$ & $\ldots$ \\
\hline
\end{tabular}

ELEMENT SIZE AND CHARACTERISTICS:

1 upper case alphabetical chasactet, full field or blank

ELEMENT DESCRIPTION:

Code to indicate other-than usual anahtical conditions or results.

\section{ACCEPTABLE CRITERIA:}

B Anaiye found in blank as well as cample. This nagking code is to be uned for analyes which are found and quantitated above the Certufied Reporaing Limir (CRL) or at highet-than-mormal beckground leveb in the mechod blank and aleo in
anaivical semples.

C Aratusis was confirmed. Thus nagring code is to be used when a confimantional

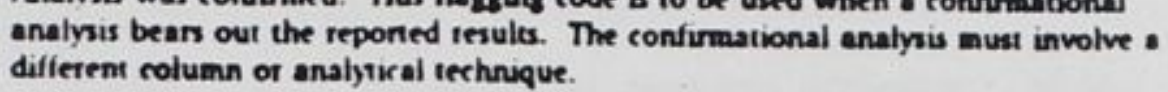

D Duplicate sample or test name. This nagsing code is to be uned to distingruish

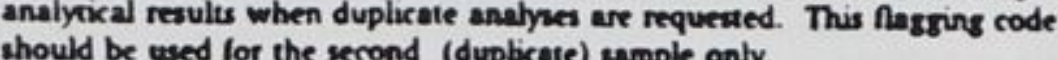

E Element run with background corrertion. This Ragging code is to be wed to identify reporied results from ICP or M analynes when beckground correction it
not the mormal mode of analysis.

F Sample filtered beloce analynis. This hagging code is to be wed when the results of fitrered samples are to be differentiated from mon-fitered samples. or when (required) finering of samples is a densanon from the sop.

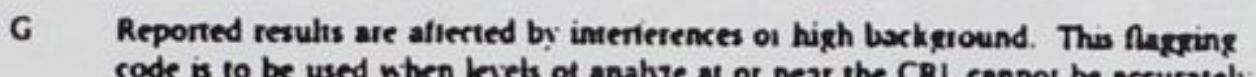
code is to be used ntren lerets of anahre at or near the CRL cannot be eccurately.
quantified to the ertual CRL due to interferents. (This will allow the laboratory it unput a different CRL Iather than defaulang to che Methods table.)

Fleoging Code

ACCEPTABle CaIteria: (CONT.)

H Sut of control but date secepted dur to high remorrnes This hageng code is to be used when control ansives show highel than normal recrovenes. assuring would have been iepored

1 Out of controt, data rejpered due to low recoveries This nagsing code is to be assurance that values ot or near the CRL are accurate.

1 Misced holding rime, acceptable based on the results of the holding - time study. This hagging code is to be used when holding timet are misted but deta is not believed to be afferted besed on ithe pont EPA.USATHuMA srudy.

k Missed holding times for extraction end preparation. This nagsing code is to be used when extraction and/or preparation dates are not met but dats qualiry is not believed to be affected.

L Mised bolding time for analynis. This hagging code is to be weed when extraction and or preparabon ames have been met bur analyocal holl

M Duplicate (high) spike analysis not mithin control lievts. This Ragsing code is to

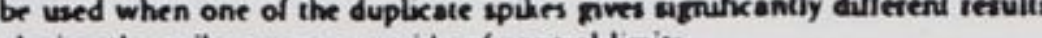
placing the spike average outside of control timits.

N Low apike rerovery is not within control linis. This hagring code is to be used Lence

Resuls less than CRL but greater then Criteris of Detection (COD). This Ragging code is to be used when the Loboratory can quancity resula which would normally tall below the CRL

Q Surrogate recovery markedly different from histoncal data. This hapring rode is 10 be weed when the reconery of a surrogate ss markedh diflerent trom historical dets.

R Analye required for reporting purposea but not currently certified. This hasering code is used to identify GCMMS analves for which no certification dats exists buit

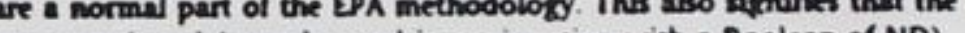
not quancitared (must be wed in conjunction wich a Bookean of ND).

2.00 Flogoing code

ACCEPTABLE CAITERIA: (CONT.)

S Results besed on internal standard. This Ragring code ts to be noed in conjunction

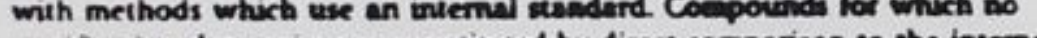

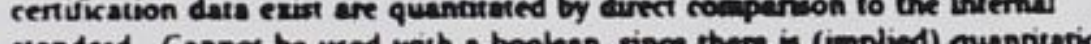

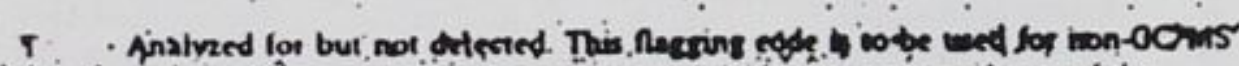
multi-analve methods to repori compoutinds that are a normal part of the methodology but for which no certification dats exisat

U Arahsis is unconfirmed. This Ragging code is to be wed when a confirmational

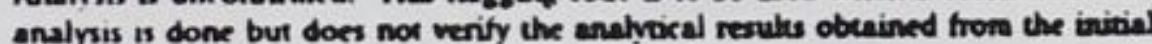
analyzis.

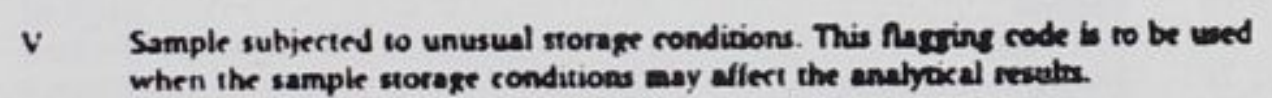

w Single ansiber Inquired from a muln-analye method. This neggring code is to be used when only one analye from a multi analyre method is to be reponed. Thi nakring code is usetw
interest tor the method.

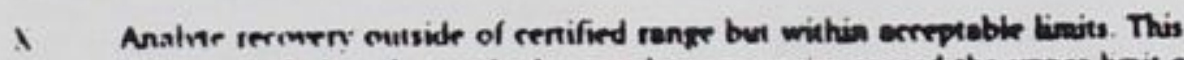

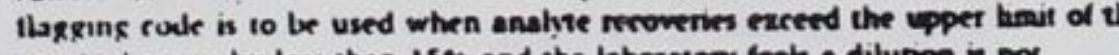
certitied range by kess than 15w and the laboratory feets o dilution is nor wananied.

ACCEPTABLE ENTRIES

B Analive found in blank as well as remple.

Analysis was confirmed.

Duphicate sompie of Test Name.

Filement run with berkground corrertion

sample fikered betore analy is

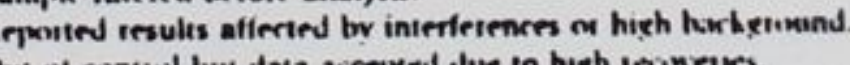

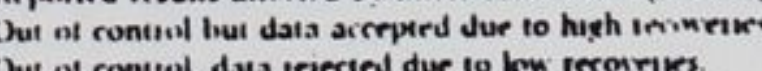

Missed holding ume, acreptabte based on holding time stud.

Missed tholding times tor extiartion and preparation.

Missed hotding time fot analyzis.

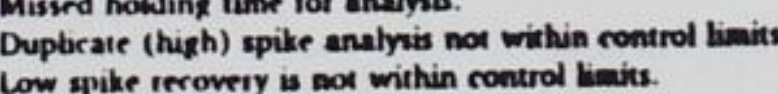

Fleoging Code

\section{ACCEPTABLE ENTRIES: (CONT.)}

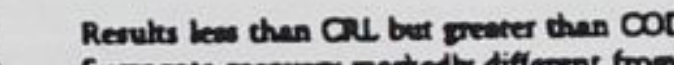

ical deter

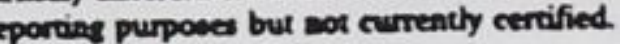

Results besed on internal mander

Nraineed lor. but.nor detectrel.

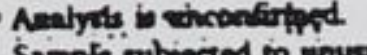

Sample subjected to unusual thoraso condition

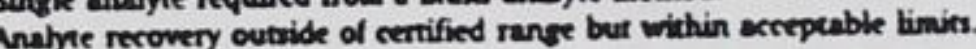




\subsection{5}

Prime Contractor

ELEMENT IS USED IN THE FOLLOWING IR RECORDS AND DATA BASE TABLES:

\begin{tabular}{|c|c|c|c|c|c|c|}
\hline nomer & 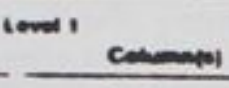 & $=$ & cond 2 & 1 & (n) & $\infty \cos$ \\
\hline tos & 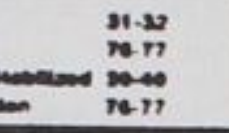 & 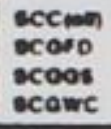 & 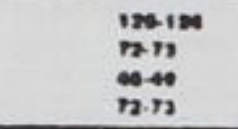 & $=$ & & $=$ \\
\hline
\end{tabular}

ELEMENT SIZE AND CMARACTERISTICS

2 alphobotic cherectere, tull fiold

ELEMENT DESCRIPTION:

Code to identify the prime contrartor (Le.e, the organization directly reeponsible to ACCEPTABLE CRITERIA

- Required on all chemical and seorechnical rerords.

ACCEPTABle ENTRIES:

(olphebotic by codo)

AC Ace Well Drilling. Inc.

AE Aetra Technucal Sernces, ins.

AG Agri Science

NH Army Environmental Hygiene Agency (AEHA)

AL Nrthur D. Linte

AN Assonne Laboratories

AP Alobams MPP

AQ Aqualab. Inc., Streamwood, II

As Alliant Teserearche. tin

AT ATEC and Associates, Ins.

BA Burget and Associates.

Betelle. Columbus. OH

BH B a H Drilling. No.

BM Bomer-Morner

CA Calron Pimber pa

soet 100

Primo Contrector

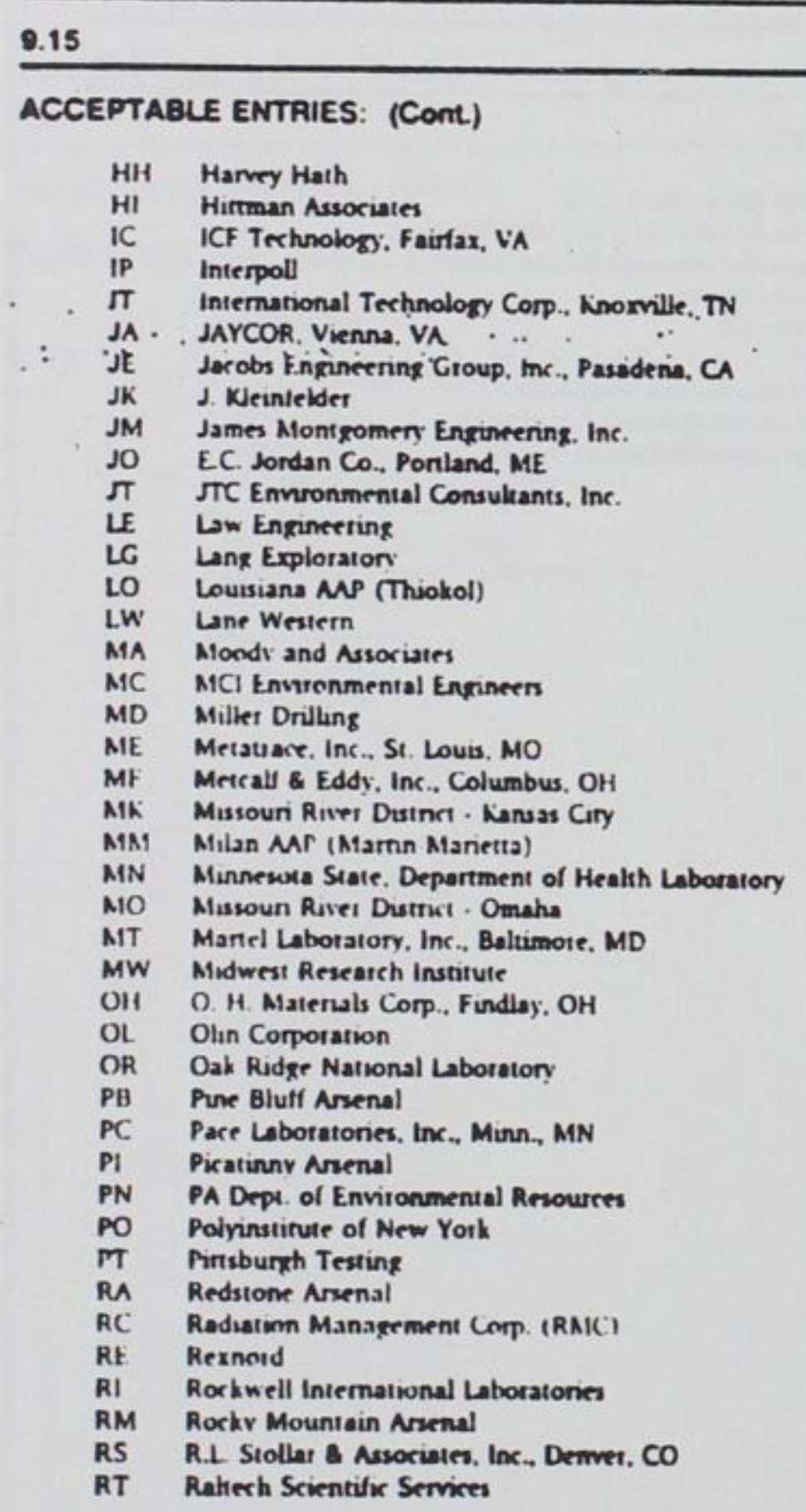

1aperd 1801

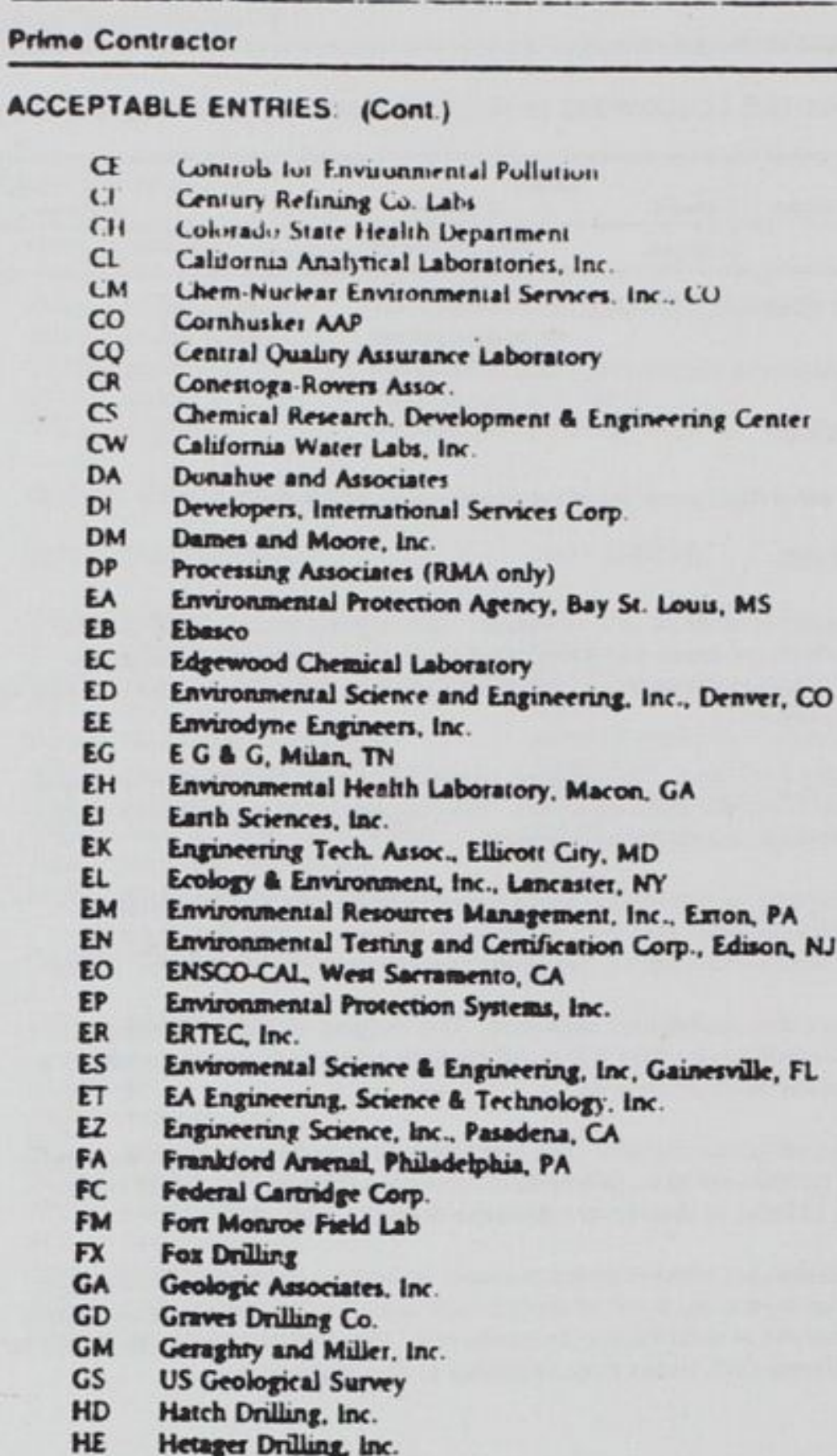

Prime Contractor

acceptable Entaies (Cont.

CE Contobs lur Enviuunniental Pullution

Cit Century Refining C. L L ats

CI Calitornis Anatyrical Laboratories, Inc

CM Chem-Nuriear Envionmental Sernces. Inc.. CO

Cornhusker Ma

Central Qualiry Assurance Loboratory

CS Conestoga-Roven Asso

Denginerring Center

DA Densahue and Asocisices

DI Developers. Imtemational Services Corp.

DM Dames and Moore, Inc.

Processing Aesocistes (RMA only)

An Environmental Protection Agency, Bay St. Lous, MS

EC Edrewood Chemical Leboratory

ED Environmental Science and Engineering. Inc., Denver, $C O$

EE Enviodyne Engineers, Inc.

EG EG \& G, Milan, TN

EH Environmental Heath Laboratory. Macon. GA

EX Eanth Sciences. Inc.

EL Ensineering Tech Assox. Ellirot Ciry, MD

EM Exology a Environment, inc., Lencaster, NY

EN Environmented Testings and Cerrification Corp., Edison

EO ENSCOCCNL, Weet Serrmenento, CA

EP ERvironmenta:

ES Enviromental Scienre \& Engineering, Inx, Gainesvilk, FL

EZ EA Enginerering. Science \& Technology. In

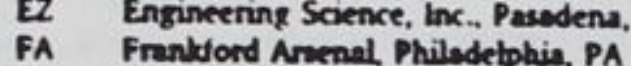

FC Federal Carridge Corp.

FM For Monroe F

GA Geologic Aesociates. In

GD Grves Drilling Co.

GS US Gerany and Miller. In

Hatch Drilling In

E Heteger Drilling ins.

Promo Contrector 0.15

\section{ACCEPTAQLE ENTRIES: (Com)}

RW Rocky Mounenia WES Combination (RMu only)
SC Shell Chemical Rocky Mountain Arenal

SD Siephenson Drilling

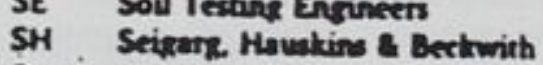

SL. Siewar Latomenonies

so. Southriver Leborator

SS STS Comsuleanar, Inc, Minneapolis, M

ST Soil Tetring Serviner

SW Swerdrup Technology. Inc.

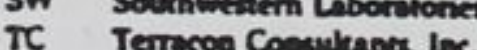

TD Tes Driting

$T \pi$ Tear Dnilivis

TH USATIAMA

in Testing. Incorporated

To Touek Avmy Depot, UT

UB DatwChem inc.

UN UNC, Grand Georgis

UT Univerniry of Teres, Atingon

VR Versar, Inc., Springtield VA

WA Water \& Nit Research, inc.

WE Waterwass Experiment Sincion (WES)

WI Werton Sences inc,

Wh. Wertion Sernces, Inc., Noreroses, GA

WN Roy F. Westom Wesidid

Weatward Opde Federal Services, Washingron, DC

WP Weet Point

WT Roy F. Weston, Inx.

WZ Warzyn Enguneering. Inx.

(bisted alphobetic thy contractor name)

Are Well Drilling. Ins.

Aetra Tecthnical Servires, inc.

Aeri Science

Nlliant Techsysems (formerty Homeymell) 


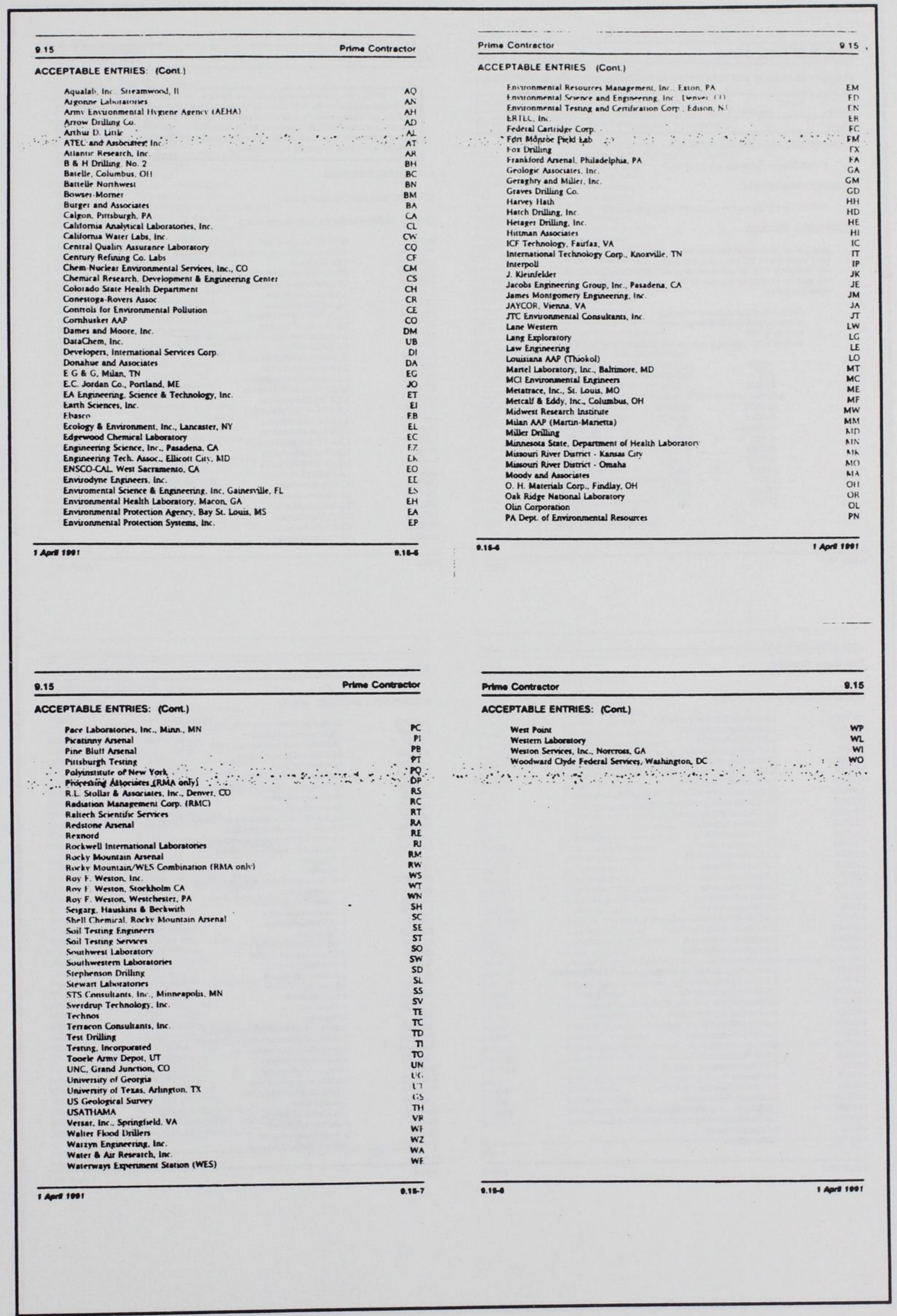


Test Name (Analyte)

8.24

ELEMENT IS USED IN THE FOLLOWING IR RECORDS AND DATA BASE TABLES:

\begin{tabular}{|c|c|c|c|c|c|}
\hline morr & Lend & nowers & 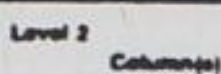 & Tollot & 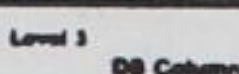 \\
\hline דינהח & $\Rightarrow$ & occins & $n$ & - & $\infty$ \\
\hline
\end{tabular}

ELEMENT SIZE AND CMARACTERISTICS:

6 alphanumeric characters, leht justified

ELEMENT DESCRIPTION:

Code to identily the analye or parameter being measured.

ACCEPTABLE CATTERIA:

Mequired on all chemical and radiological records

For untenowns, musur be within the range of UNKOOI through UNKoge

Lab must be certified for the apecific Teat Name eucept when one of the lollowing conditions exists:

Method is semiquantitative screening

Method is

\begin{tabular}{|c|c|c|}
\hline ACDIT & $\infty 00$ & REACTY \\
\hline $\begin{array}{l}\text { NUK } \\
\text { NLKBIC }\end{array}$ & $\begin{array}{l}\text { CU, } \\
\text { COLIOR }\end{array}$ & $\begin{array}{l}\text { SAUNAE } \\
\text { SNUNN }\end{array}$ \\
\hline NUKCAR & COND & ssol. \\
\hline NLKFYD & CORRT & TASTE \\
\hline NLKPHE & CROCO & TOS \\
\hline ALPHAG & DO & TEMP \\
\hline AMOS & DOC & TOC \\
\hline ANPHO & EPTOX & TOX \\
\hline ASBEST & HARD & \\
\hline BETAC & IGNIT & TREACT \\
\hline$B O D$ & ODOR & TSOUD \\
\hline CHARD & OILGR & \\
\hline CHRYS & PH & TURBID \\
\hline
\end{tabular}

1 Apont 1001

Toet Namo (Anetyo) 2.24

ACCEPTABLE ENTRIES: (Coml)

12DNAP

$120 \mathrm{PPH}$

12EPCH

12EPEB

$12 M T D M$

$127 \mathrm{MCP}$

$135 \mathrm{MCP}$

$135 T$ TNB

$13 C P D O$

130804

$130 \mathrm{DC}$

$13 D C P$
$13 D C P E$

13DEB

13DFB

13DMM

13DMCH

$13 D N A P$

13DNB

13DPPR

$13 \mathrm{MCP}$

$13 \mathrm{MCPE}$

$1402 E \mathrm{E}$

14DAC

140804

$140 \mathrm{DB}$

$140 \mathrm{DC}$

$140 F B$

14DMB

14DMCH

14DMNP

$140 \mathrm{~m} \times$

14DNB

14001 1001
1.2. Dimerthytbenzene / o-xyled

1.2. Dipherey

1.2. Diphenenylhydraxion

Cyclohexene oxide / 1.2-Eporyeyclohesene

1.2. Eporyethylbenzene /

1.2.2. Tetramethylkelopropan

1.3.5. Trimethyloenzener

3.5-Trinitrobensene

1.3. Creclopemiodione

3. Dichlorobenaene

3. Dichloropropane

3.-Diecthylbensene

3-Dimathylbenrese / I X xvilene

1.3. Dimethylbury) benzene

3. Dimethylocioherane

3.-Dimethylaph hehaleen

1:(1.3.-Propanediyl) bis (benzene) / 1.3-Dtphenytpropene

1.3-Dihydro-2H-indol-2-one

13. Tetradecynoic ecid, methyl ester

1.4-Diacerylbenzene

1.4-Dichlorobenuene-D

1.4-Dichlorobensene

4.Dilloorobenzen

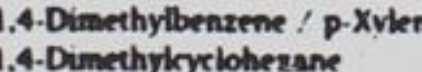

1.4-Dingdro-1.4-methanonaphithalen

1.4-Dimethoryanthracene

1,4-Dinitrobenseace
12. Mechyletraderanaic acid merhyl eater
6.24

Toet Nemo (Analyo)

NOTE: For unknown compounds, use the code UNLOOOC where 2000 represents the number ascigned by the field leb to the unknowns from 001 thru 999 . The numbern are full field, 00 "unknown one" would be expreased at UNKool." with the refos inctuded. The documentation and be consistent within the cume installation. Therefore UNKoO 1. Can only represent one unique known for each instollation

ACCEPTABLE ENTRIES:

Chomiced and Radlotogical Data:

(Sortad alphebotically by Teet Nemo code)

OINHCL.

$10 \mathrm{CUDM}$

$0.1 \mathrm{~N}$ Hydrochloric acid

$10-$ Crelopentylundecanoic acid, methyl ener

1004 Mechenol

10-mechylundecanoic acid, methyl enter

IOMUDM

1OOEME

IIITCE

1,1,3 Trimethykycloheran

IDCE

IDCE

IIDMEB

IMCPE

234MB

$23 C P R$

$123 M$ CH

$123 \mathrm{TCB}$

$123 \mathrm{MMB}$

$124 M C H$
$124 T C B$
$124 T M B$

$247 \mathrm{MB}$

DDERE

$12 D C D 4$

$12 \mathrm{DCE}$

$12 \mathrm{DCOB}$

1,1-Dichloro-1-propene 1 1.-Dichloroethylene / 1,1-Dichloroethen

1,1-Dichloroethane

1,1-Diphectyylethyl) benthe

1.2.3. Teras chencane

12,3-Tichloropropene

1,2,3-Trimethyloycloberas

12.3. Trichlorobenzene

1.2.3-Trimechylbenzene

1,2, Trimecthyloyedoberan

1.2. Thimerobenesene

1.2. Dichlonobemenae- Dor

1.2-Dibromoecthane

1.2-Dichloroecthane-DA

1.2-Dichloroechenes / 12.Dichloroechylenes (cis and trans iromers)

12.Dichlorobentene

1.2-Dichloroethane

ans

14001

.24

Toet Namo (Anelyno)

ACCEPTABLE ENTRIES: (ComR)

14MPME 14.Mechytpentadecanic add, mechyl eter

STMHME

16DMIN

6DNNP

6MAHIME

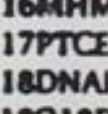

OOIRD

N3MP2

BY4H:

ICAL
ICDL
ICDMZ
ICH

$\mathrm{CH}$

a.24h

ICONA

$1 D O D C$

IE24DB
IE2MB

IEZMB

IEFB

IFNAP

IHPDOL

IHXE

IMRPEC

IMTMEN

IMCFNE

IMDB

IMECPR

IMFUR

IMNN

IMENB

5-Methylheredecanoix acid, methyl eover

1,6-Dimethylinden

1,6-Dimethylaephathaleme

16-Mecthylhepredecanoik acid, methyl erer

17.Pentetrieromem

8. Dispethylnephethelene

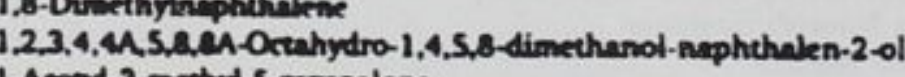

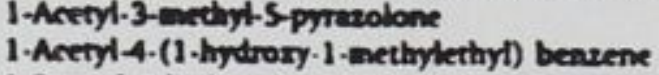

1-Benzyt-4 hydromteninitarate

I.Propanol

1. Butanol

1-Carbemoyl-3.5-dimethyl-2-pyrazoline

1.Chloroherane

Chloro-2,4-heredien

1-Chloromaphilhalene

1. Doderanol

1.Ethyl-2,4-dimethylbenzem

1.Ethyl-2-methylbenzene

1. Ethylherylbensene

1. Ethylpropylbenserse

1. Fluoronephthaim

-Heren-3-ol

-Methyl-2-(2-propenyl) extopeniame

-Methyl.7. (1-methylethyl) naphthalene

1. Methylbens (N) anchrocem

1. Methylidectopentbensene

Methperybrazere

. Methylethyleystopropan

- Methylinden

1.-Methyl-9H-Aworeme

1-Methylnaphithalene

Methylnonylbemeene 
Teet Name (Anolyte) 0.24

ACCEPTABle EntRies (Cont)

\begin{tabular}{|c|c|}
\hline IMPYR & 1. Methvipurene \\
\hline $\begin{array}{l}\text { IMXIPF } \\
\text { IN2ONF }\end{array}$ & $\begin{array}{l}\text { 1 Nethoxy 1 propene } \\
\text { 1. Nutto-2.octanone }\end{array}$ \\
\hline INAPA & $\begin{array}{l}\text { I Naphehviamine } \\
\text { intane }\end{array}$ \\
\hline INHP & I Nutroheptaner \\
\hline INKCL & 1. ON Polassium chionde wolution \\
\hline INPN & 1-Nitroptopane \\
\hline IOCTOL & 1.-Ortanol \\
\hline IPECHX & 1. Propenylcycloterane \\
\hline IPNAP & 1. Phenylnaphithalene \\
\hline ІТВСНА & 1.1-Burykecloheranecartorylic ecid \\
\hline 210DMU & 2.10-Dimethylundecane \\
\hline $2255 C B$ & 2.2.5.5-Tetrechlorobiphenyl \\
\hline 225TCB & 2.2.5-Trkhlorobuphenyl \\
\hline $226 \mathrm{TMO}$ & 2.2.6. Trimecthyloctene \\
\hline 22DMCA & 2.2. Dimethylbutane \\
\hline $2345 C B$ & 2.3.4.5-Tetrachiorobiphenyl \\
\hline $2346 C P$ & 2,3.4.6-Tetrachlorophenol \\
\hline $2356 C P$ & 2,3,5,6-Tetrachlorophenol \\
\hline $235 T C P$ & 2,3,5-Trichlorophenol \\
\hline $235 T M D$ & 2.3.5-Trimethyldecane \\
\hline $236 \mathrm{TMN}$ & 2.3.6-Trimethylraphethalene \\
\hline $237 \mathrm{TMO}$ & 2,3.7. Trimethylociane \\
\hline 23CIPE & 2.3-Dichloro-1-propene \\
\hline 23D2HL & 2.3-Dimethyl.2-heranol \\
\hline 23DCLP & 2.3-Dichlorophenol \\
\hline 23DMC4 & 2.3-Dimethylbutane \\
\hline 23DMCS & 2.3-Dimethylpentane \\
\hline 23DMP & 2,3-Dimethylphenol \\
\hline 23DNAP & 2,3-Dimenthytraphthalene \\
\hline $237 M P$ & $2,2,3,3$-Terrmethytpentane \\
\hline 245PCB & $2,24,5,5$ - Pentechlorobiphenyl \\
\hline 245T & 2.4.5-Trichiorophenoxyaceric arid \\
\hline $245 T C P$ & 2,4.5. Trichlorophenol \\
\hline 245TP & 2.(2.4.5-Trichlorophenory) Propionic \\
\hline $246 \mathrm{MPY}$ & 2,4.6-Trimethylpyridine \\
\hline $246 \mathrm{TBP}$ & 2.4.6-Tribromophenol \\
\hline $246 \mathrm{TCA}$ & 2,4,6-Trichloroaniline \\
\hline $246 \pi C P$ & 2.4.6-Trichlorophenol \\
\hline $246 \mathrm{TMO}$ & 24.6-Tnmethyloctane \\
\hline $246 \mathrm{TNP}$ & 2,4,6-Trinitrophenol / Picric acid \\
\hline
\end{tabular}

inent reer

enerarbontic ecid

2.5.5-Tetrechlorobiphenyl

2., $4,5,5$. Pentachlorobiphen

2.4. Tninchorophenoxy

2.4.5-Trichlorophenoxy) Propionic Acid

2,4,6-Trinitrophenol / Picric ocid

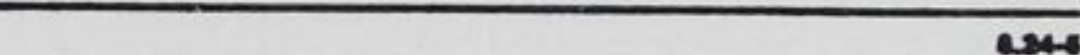

aCceptable entries (Cont)

24nTNR 2.4. Trnitroresmenol / Sryphnk acid

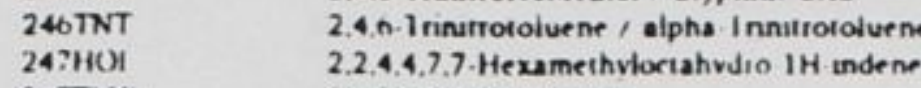

24 TMC) 2.47 Tnmethitociam

24) 2.4. Dichlorophenorvacetic acid

$24 \mathrm{DB} \quad$ 4. (2.4. Dichlorophenoxv) burync acid / 2,4.DB

240 24DCLP 2.4 Dichlorobiphenst

$\begin{array}{ll}240 C L P & \text { 2,4. Dichlorophenol } \\ \text { 24DMCS } & \text { 2,4-Dimethylpentane }\end{array}$

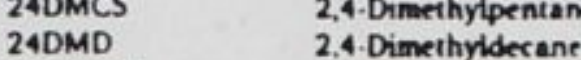

24DMHX 2,4 Dimethylherane

$\begin{array}{ll}\text { 24DMPN } & \text { 2,4.Dimethylphenol } \\ \text { 24DNP } & \text { 2.4.Dinitrophenol }\end{array}$

$\begin{array}{ll}\text { 24DNP } & \text { 2.4. Dinitrophenol } \\ 24 \mathrm{DNT} & \text { 2.4. Dinitrotoluente }\end{array}$

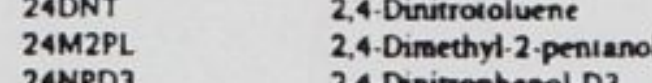

24.4. Trimephenol D3

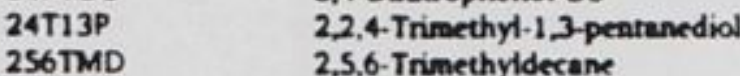

2SC14D 2,5-Cyelohethydiecen-1,4-dio

25DCMP 2.5. Dichlorophenol

$\begin{array}{ll}\text { 25DMP } & \text { 2.5. Dimethylphenol } \\ \text { 25DMPA } & 2,5 \text { Dimethylphenenthrene }\end{array}$

2SETHF 2.5-Dimethyltetrahudroluran

2SHPCB $2,2,3,4,5,5,6$ - Heptachlorobiphenyl

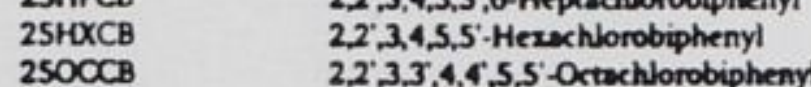

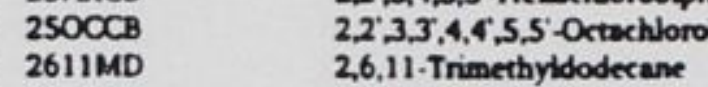

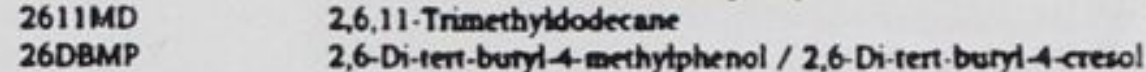

$260 \mathrm{MP}$

26DMO

26DMP

26DMST

260NA

260NT

$26 \mathrm{HPCS}$

$27 D N A P$

29DMUDA

2,6-0ichlorophenol

2,6-Dimethylphenol

2,6-Dimechylsyrene

2,6-Dimethylundeca

2,6-Divicroeniline

2.6-Dinitrotoluene
$2,2,3,4,4.5,6-$ Heptachlorobiphenyl
2.7. Dimecthyloctane

27.Dimethyloctane

2.9. Dimethylundecterane

2-Amino-1,6-dinitrouniline
Toet Namo (Anelyne)

8.24

\section{ACCEPTABLE ENTRIES: (Cont)}

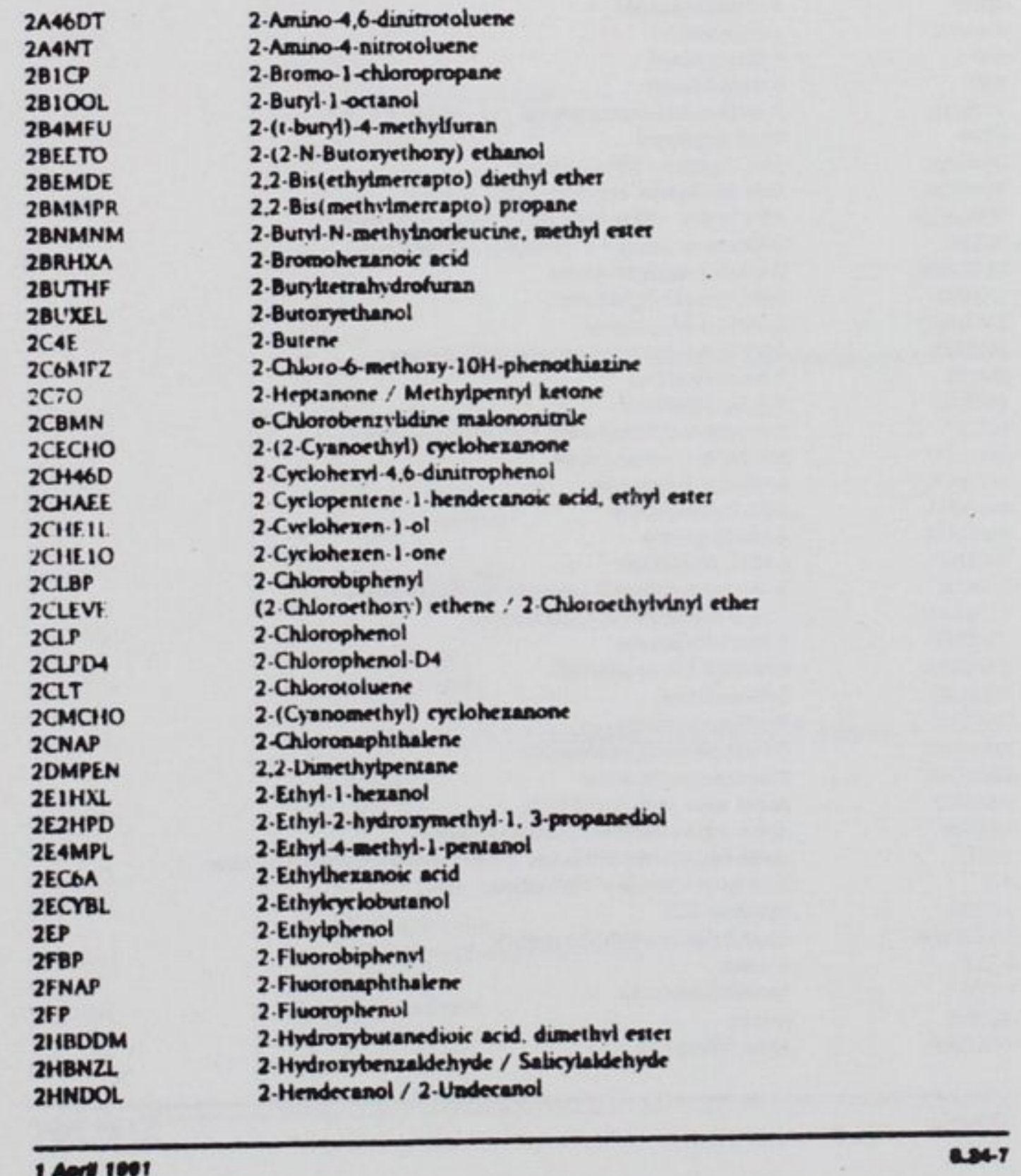

and

2.24

Toet Nomo (Anetrye)

ACCEPTABLE ENTRIES: (Cont)

2HMP 2 2.Hytrontiphent

2MIDDL 2 2-Methyl1-1.dodecanol

2M24P 2 2.Methyl.2.4-pentanediol

$2 \mathrm{M} C \mathrm{C}$

$2 \mathrm{M} 2 \mathrm{H} 3 \mathrm{~B}$

2M3HXE

$2 \mathrm{MBZ}$

$2 \mathrm{MC}$

$2 M C^{2 M C}$

2MC7

$2 \mathrm{MCPL}$

2MDOD

2MENAP

2MEPEN

2MMECO

2MNAP

2MPAIE

2MPNHT

2MPAME

2MPEAE

2MTETD

$2 M T M A F$

2MXIPE

$2 \mathrm{MPESC}$

$2 m \times T M$

2N3C

2NANA

2NKCL

2-Mechyl-2-propanol / iert-Butanol

2-Methyl-2-hydroxy-3

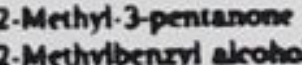

2-Mechylpropene / trobutane

2.Methylbutane / bopencane

2. Methylherane / boheptane

2. Methylheptane / bookine

2.Methylky clopentano

-Methyldodecerne

2.-(1-Methylecthvi) naphrthalene

2-Methylocteder anok axid

2-Methylpentare

2.Methylphenol / 2 - Creepl

2-1soburynx acid

-Methytpropenoic arid, methyl enter

2.Methyl-2-propenoik acid, 1.2-ethanediyl eater

2.Mechylpyrene

2. Methyterradecane

2-Mechylthio 4 - hydrompyrimidine

2.-Methary-1-propene

2-Methoxy-2-methylpropane / ien-Buntmethyl ather

3-Mechyl-2-nitrophenol / 2-Nuro-merresol

2. Nitroeniline

2-Napthinylamine

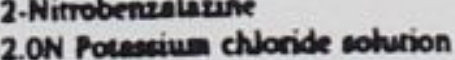

2.240

Inent 1001 


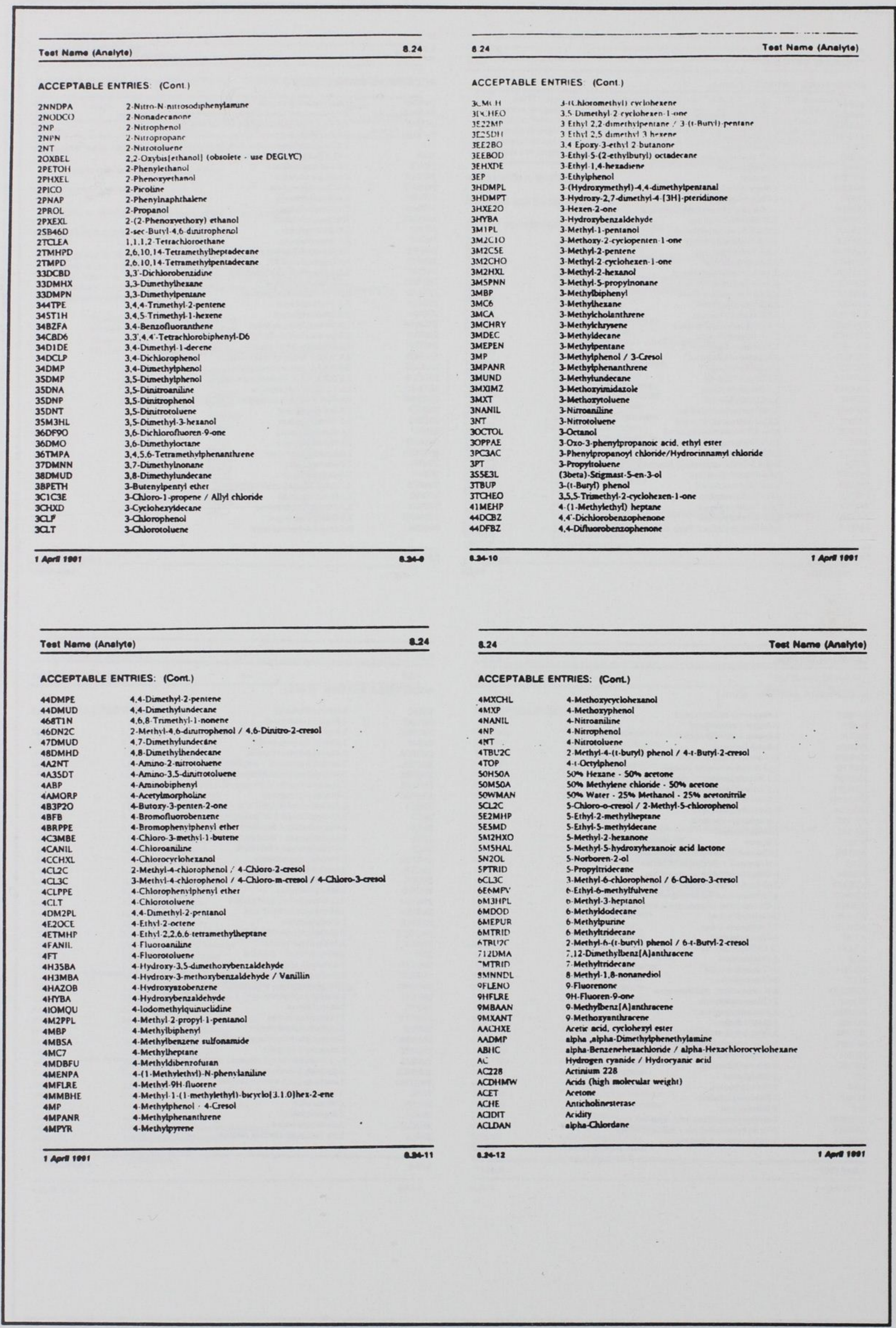


Toet Namo (Anolyto)

6.24

ACCEPTABle ENTRIES (Cont)

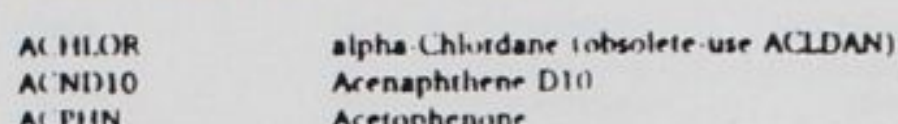

ACPIIN Acetophen

ACRILO - ACronionite

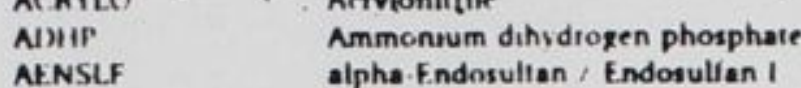

AG $\quad$ Silver

Al. Numinum

ALDEHY Niphatic al

$\begin{array}{ll}\text { ALDRN } & \text { Ndinn } \\ \text { ALHC } & \text { Niphanc hudrocantons } \\ \text { ALHMW } & \text { Neohols (high molecular weight) }\end{array}$

Nrohols

ALKBIC N Nealinity-bicarbonale

ALKHYD N Nikalinity - hydroxide

$\begin{array}{ll}\text { ALKN } & \text { Nkanes } \\ \text { ALKPIE } & \text { Nkalinify - phenolphthalein }\end{array}$

NLPGF Npha gross-field

ALPGLA N N ha gross- boluble acid fraction

Nphe grose-solute woter frocion

NLPHAC NPho gross

AMPHPN $\quad$ Alpho-Prnene

AMOS Nmosite astestor

NAPNE Acenaphthene

Acenaphthylen

ANELNT Anion elle

ANPHO Anthophyllite asbestios

ANTRC Anthracene

ANTRCN 9-Anthracenecarbonitrite

ANTRQU 9.10-Anthracenedione ; Athraquinon

AS ANEenic

ASBEST Astestos
ASEXT Arenic extractable

2.4.6. Trinitrobenseldehyde

1apent 1901
02419
824

Toal Neme (Analye

ACCEPTABLE ENTRIES (Cont)

ATNT Alpha Trinitrotoluene fobsolete ure 246TNT

Atmine
Gold

ATISTH NACN Nivi ether

NJACN Azirylononane

B

B2CLEE Bis (2-ehloroethyl) ether

B2EHP Bis (2-ethylhexyl) phithalate

$\begin{array}{ll}\text { BA } & \text { Barium } \\ \text { OMUNTR } & \text { Benzo[A]anthracene }\end{array}$

$\begin{array}{ll}\text { BANTR } & \text { Benzo(A)anthracene } \\ \text { BAC } & \text { Benzal chlonde } \\ \text { BNIXE } & \text { Butanoic acid, 1-hexyl ester }\end{array}$

$\begin{array}{ll}\text { BNIXE } & \text { Butanoic acid, } \\ \text { BAPYR } & \text { Benzo(A) A prene }\end{array}$

BBFANT Benzo(B) Invorenthene

BFLRE Benzo(B) fluorene

Benzo(B) lluorene

beta-Benzeneheracthoride / thera-i

Benzo(B) naphtho(2,3-D)luran
Benzo(B) naphtho(1.2-D)thiophene

Burylbenzyl phitholate

Bicrcto $(2,2,1)$ heptes-2.5-dien

bets-Chlordone

Bis (chloromechyl) ether

Bis (carboxymethyl) sulfoxid

Bis (carboxymethyi) sulione

(DDT reethyled)

Bicyclo $(3,1,0)$ heren

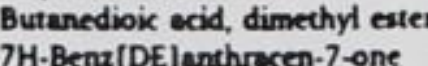

Beryllium ,

Beryllium 7

1-(2-Butoryethory) ethano

Beta gamma gross

Gela Endosulian /

Bensianthrone

Benzidine

2-Butoryethenol phosphat
Toet Name (Anelyte)

.24

\section{ACCEPTABle ENTAIES: (Cont.)}

\begin{tabular}{|c|c|}
\hline $\begin{array}{l}\text { BEPYR } \\
\text { BETAG }\end{array}$ & $\begin{array}{l}\text { Benzo[E]pyine } \\
\text { Bets gross }\end{array}$ \\
\hline BETGF & \\
\hline BFTGL. & Beta gross-lab \\
\hline $\begin{array}{l}\text { BETGLA } \\
\text { BETGLW }\end{array}$ & $\begin{array}{l}\text { Beta grpss-woluble and fraction, } \\
\text { Beta gross-roluble water fraction }\end{array}$ \\
\hline BF2ANT & $\begin{array}{l}\text { Beta grosti-oluble water traction } \\
\text { Bensobilluorosnthene. }\end{array}$ \\
\hline & BenzolG.H,IInuroanthene \\
\hline $\begin{array}{l}\text { BGHIPY } \\
\text { BHC }\end{array}$ & Benzo(G,H,I)pentene \\
\hline $\begin{array}{l}\mathrm{BHC} \\
\mathrm{BI}\end{array}$ & $\begin{array}{l}\text { BHC. nonspecific } \\
\text { Busmuth }\end{array}$ \\
\hline 81212 & $\begin{array}{l}\text { Bumuth } \\
\text { Bismuth } 212\end{array}$ \\
\hline 81214 & Bismuth 214 \\
\hline Bicvix & Bucretohervl \\
\hline RIOAI & 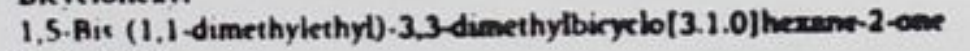 \\
\hline BINAP & Binaphethyl \\
\hline BHFANT & Benzo; J) fluoranthene \\
\hline BKFANT & Benzo(K) fuoranithere \\
\hline $\begin{array}{l}\text { BLDX } \\
\text { HMIr }\end{array}$ & Bladex \\
\hline BMD & Buntmethyl phithalase \\
\hline BOD & Biological oxyzen demand \\
\hline BOLS & Bolstat \\
\hline $\begin{array}{l}B P B C \\
B R\end{array}$ & Burytphthatyl butiglveolate \\
\hline $\begin{array}{l}\text { BR } \\
\text { BRCBHS }\end{array}$ & $\begin{array}{l}\text { Bromide } \\
\text { Bromubenzene }\end{array}$ \\
\hline BRCLM & $\begin{array}{l}\text { Bromulenzierie } \\
\text { Bromorhlorumethane }\end{array}$ \\
\hline BRDCINI & Bromodkhloromethane \\
\hline ВRMCIL. & Bromeril \\
\hline BTAZON & 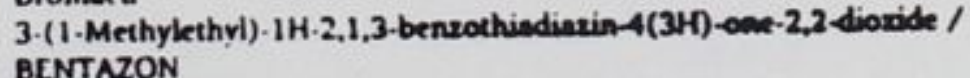 \\
\hline & $\begin{array}{l}\text { BENTAZZN } \\
\text { Benzotrichloride }\end{array}$ \\
\hline BTMSOA & Bus (trimethylaibl) oxalix acid \\
\hline BTZ & Benzothuarole \\
\hline Bucolls & Burythensene \\
\hline BUEETH & Burylethyl ether \\
\hline & \\
\hline BZNL2M & $\begin{array}{l}\text { alpha, alpho-Dumethribenzenemecthanol } \\
\text { Benzvi alohol }\end{array}$ \\
\hline 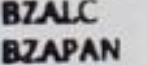 & \\
\hline BZCPAN & Benzol Clphenenthrene \\
\hline BZFANT & Benefluoranthene \\
\hline
\end{tabular}

iand 1001
2.24

ACCEPTABLE ENTRIES: (Com)

BZHOUN Benzo(H)quinotine

Benzoic acid, methyl ester / Methyl benzoote

(1)

2OTRP

ZZTRZ

BZPA

Brm

Ci1

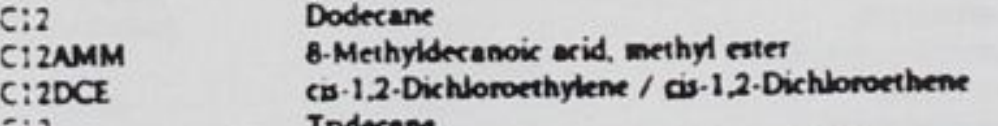

$\mathrm{C}: 3$
$\mathrm{C}: 3 \mathrm{DCP}$

C13

C14

cianse

C:S
CisA
C:0

C: 104

C:OABE
S:OADM

C:OADM

C:OAME
CIOSAT

ci:?

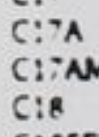

C:INM
CIBSFP
CISA

CIGABE
CIONE
CIBAME

CIOAME

Cistod
Cisuns

C18UN
C19
C19A

Benzoic acid ammonium as

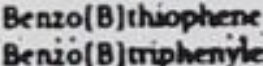

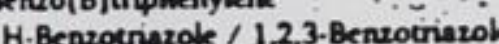

Benzenephosphonix arid

Benzyl bromide / alpha-Bromorolvern

Derane

cs-1,3-Dichloropropylene / cis-1,3-Dichloropropene

Terndecane

Tetradecanoic acid / Myristic acid

Pentadecane

Pentaderanoic acid

Hersdecane

Herodecanoik acid / Palmirik acid

Heraderanoic acid, bumethyl etert

Heraderanoic exid, bis (2ethylheryl) ester

Sorvutated hydrocobons (C16)

Hepraderane

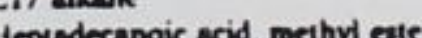

Heppodecanoic

Bu (pentaffuorophenyl) phemyl phosphime

CI8 alleane

Ortadecanoic arid. burth ester

Ortaderanoic and. ensil ester

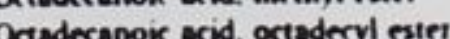

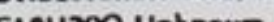

Nonaderanoix acid 
Toet Nome (Anatyo)

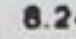

\section{ACCEPTABLE ENTRies (Cont.)}

\begin{tabular}{|c|c|}
\hline CIADMF & Carbonic acid dimethyl ester \\
\hline & Eirosane \\
\hline C21 & $\begin{array}{l}\text { Heneremane } \\
(2211400 \text { Lnknown }\end{array}$ \\
\hline C22LIN & $\begin{array}{l}\text { (2211400 Lnknown } \\
\text { Penvarosente }\end{array}$ \\
\hline CZAEE & Aretk acid ethyl esiter ; Ethyl ocerate \\
\hline C2AVE & Acetic ocid. nnyl estet / Vinyl acetate \\
\hline $\mathrm{C} 2 \mathrm{H} 3 \mathrm{CL}$ & Chioroethene / Vinyl chlonde \\
\hline C2HSCL & Chloroethane \\
\hline CЗОAME & Tnacontanoic acid, methyl ester \\
\hline C35 & Pentatrucontane \\
\hline $\mathrm{C} 36$ & Hexatriacontane \\
\hline С $32 \mathrm{MMB}$ & Propanoik acid. 2-methviburyl ester \\
\hline СЗАME & Propanoic acid. methvl ester \\
\hline & Butane \\
\hline CAHXIL & cis-4.Heren $1-01$ \\
\hline & Pentanoic acid / Valeric acid \\
\hline$C O D 6$ & Benzene. Do \\
\hline $\mathrm{COH} 6$ & Benzene \\
\hline СОНОН & Cyclohexanol \\
\hline C7 & Heptane \\
\hline C7A & Heplanoir acid \\
\hline C7NBI & Hepiachioronorbornene \\
\hline$C_{B}$ & Ortane \\
\hline & Cis alkane \\
\hline COAME & Ortanoic arid, methyl eater \\
\hline$c 9$ & Nonane \\
\hline cu & Cakium \\
\hline СМн & Chloroscetaldehyde \\
\hline CACOZ3S & Calcium carbonate solution \\
\hline СNLMW & Hydrocarbons (all molecular weighes) \\
\hline CAMBEN & 3-Amino-2.5-dichlorobenzoic acid / CHLORUMBEN \\
\hline CAME & Carbamix acid, methvi estet \\
\hline самр & Comphor \\
\hline CAPLCT & Cappolortam / O-Aminohexamoix arid lartam \\
\hline CARBAZ & 9H-Carbazole \\
\hline CATOL & Caterhol \\
\hline & a-Chiorobenzaldehwe \\
\hline $\mathrm{CBCCH}$ & cis-1-Bromo-2-shlorors \\
\hline CBOA & ochlorobenzoir acid \\
\hline$\infty$ & $x \times 0 \mathrm{C} 3$ \\
\hline
\end{tabular}

ind 1001
6.2

Toet Nome (Anelyto)

ACCEPTABle ENTRies: (Cont.)

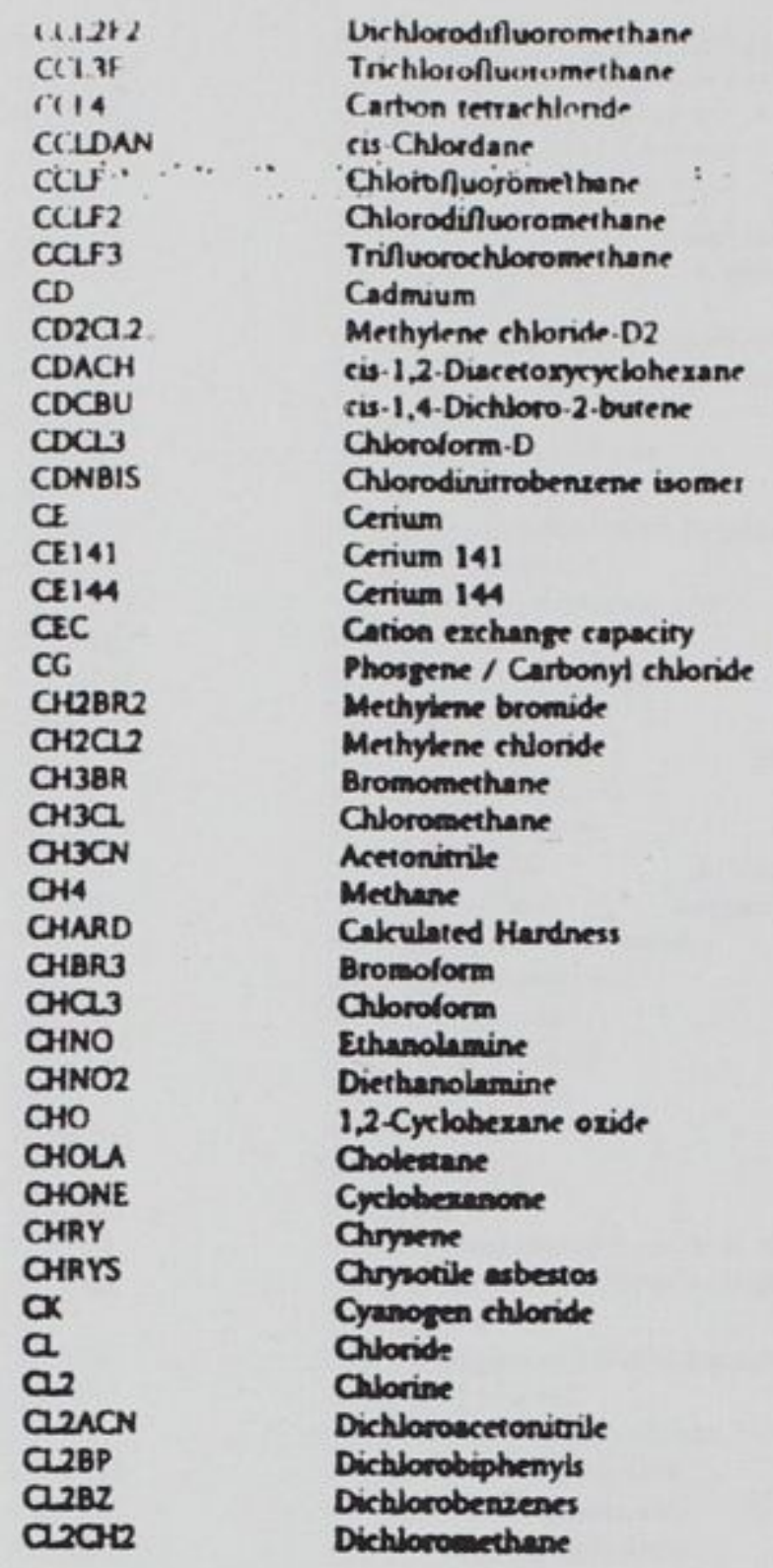

Teet Namo (Analyne)

224

ACCEPTABLE ENTRIES: (Comt)

\begin{tabular}{|c|c|}
\hline C.2NAP & Dichloronaphthalenes \\
\hline CL3BP & Trichlorobiphenyls \\
\hline CL3C3E & Trichloropropenes \\
\hline CL3NAP & Tricbloronaphchalenes \\
\hline & -Tinchlorophignals : \\
\hline CABp & Tetrachlorobiphenyts \\
\hline CLANAP & Tetrachioronaphishalenes \\
\hline CLSB & Peniachlorobenzene \\
\hline CLSBP & Pentachlorobiphenyls \\
\hline CLSET & Pentachloroethane \\
\hline CGRP & Mezechlorobiphenyts \\
\hline CoBz & Hezachlorobenzene \\
\hline CGCP & Herachloroxyclopentadiene \\
\hline CLEET & Hexachloroethane \\
\hline C.7BP & Heptachlorobiphenyls \\
\hline C.7NB & Hepeachloronorbormediene, \\
\hline$C_{C L}$ & Chloroeretic ecid \\
\hline CLCODS & Chlorobentene. DS \\
\hline CLCOHS & Chiorobenzene \\
\hline CLCHIX & Chlorocyeloherane \\
\hline CLD & Chionne demand \\
\hline CLOAN & Chlordane \\
\hline CLEN & Chlordene \\
\hline CINAP & Chloronaphithalenes \\
\hline $\mathrm{CO}_{3}$ & Chiorate \\
\hline ap & Chlorophenols \\
\hline CIVRA & 2. Chlorovinyl arnonic acid \\
\hline CUXB & Chlonnated bensenes \\
\hline CUXNAP & Chlorinated naphathelenes \\
\hline CMME & Chloromethyl methyl ether \\
\hline CMONOX & Carbon monoxide \\
\hline $\mathrm{CN}$ & Chloroseretophenone \\
\hline co & Cobalt \\
\hline $\mathrm{CO} 2$ & Garbon dioride \\
\hline$\infty 3$ & Corbonate \\
\hline $\cos 7$ & Cobaht 57 \\
\hline co6o & Cohali on \\
\hline COD & Chemical orvgen demand \\
\hline $\mathrm{cou}$ & Fecal coliform \\
\hline COLOR & Color \\
\hline COND & Specific ronductiving \\
\hline
\end{tabular}

14001 1001

\section{ACCEPTAQLE ENTRUES: (Com)}

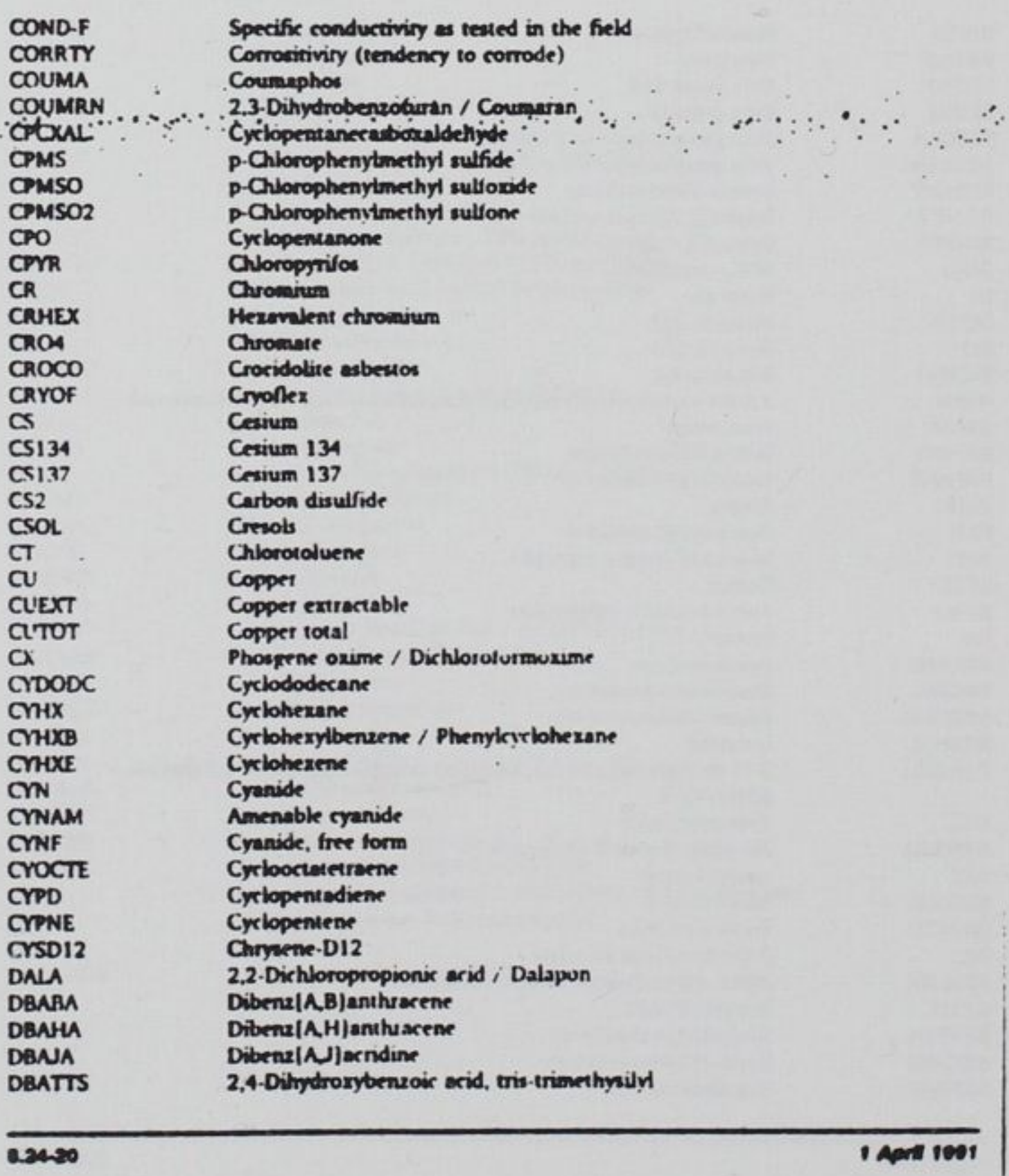


Toet Neme (Analye)

8.24

ACCeptable entries: (Cont.)

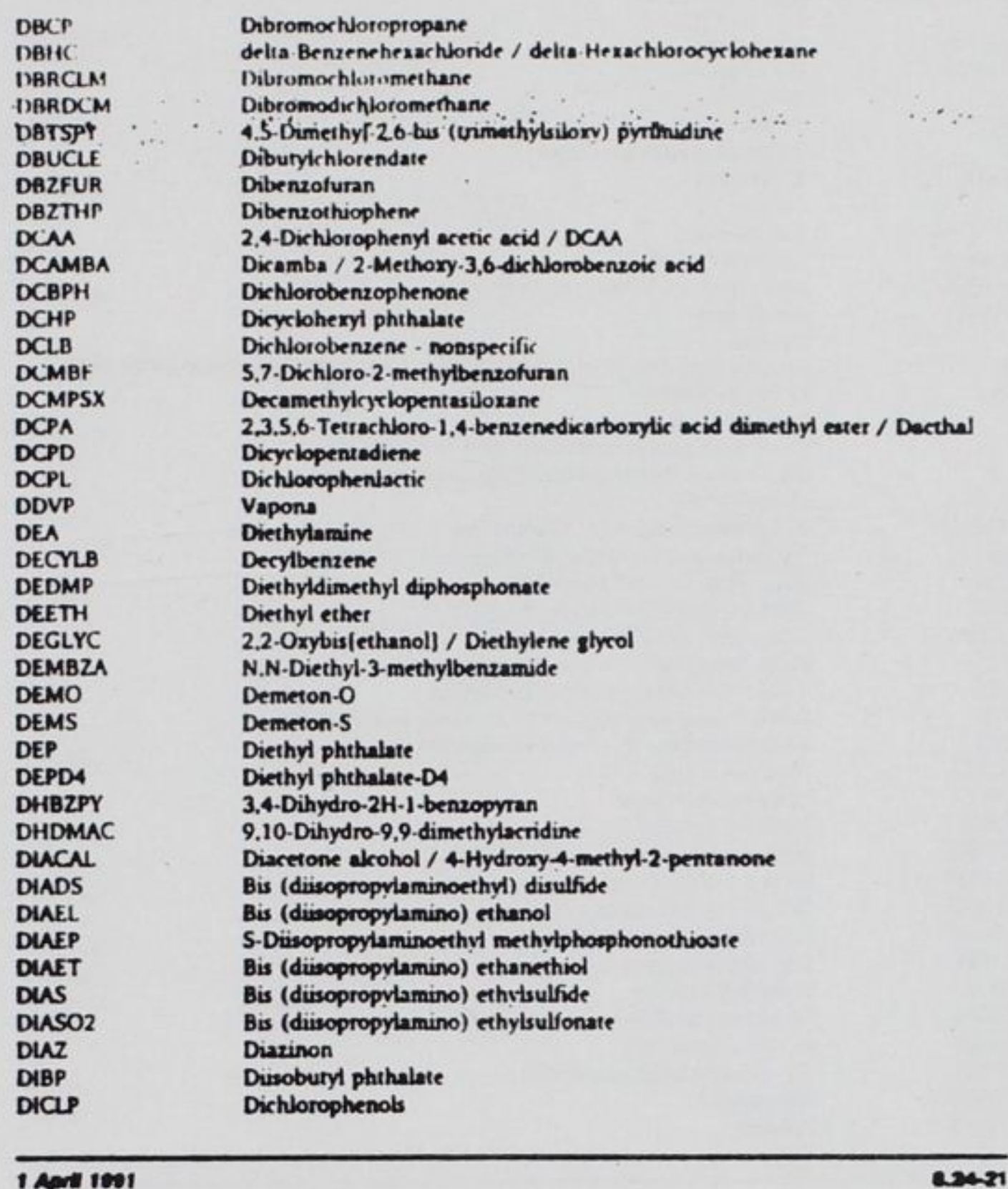

8.24

ACCEPTABLE ENTRIES (Cont)

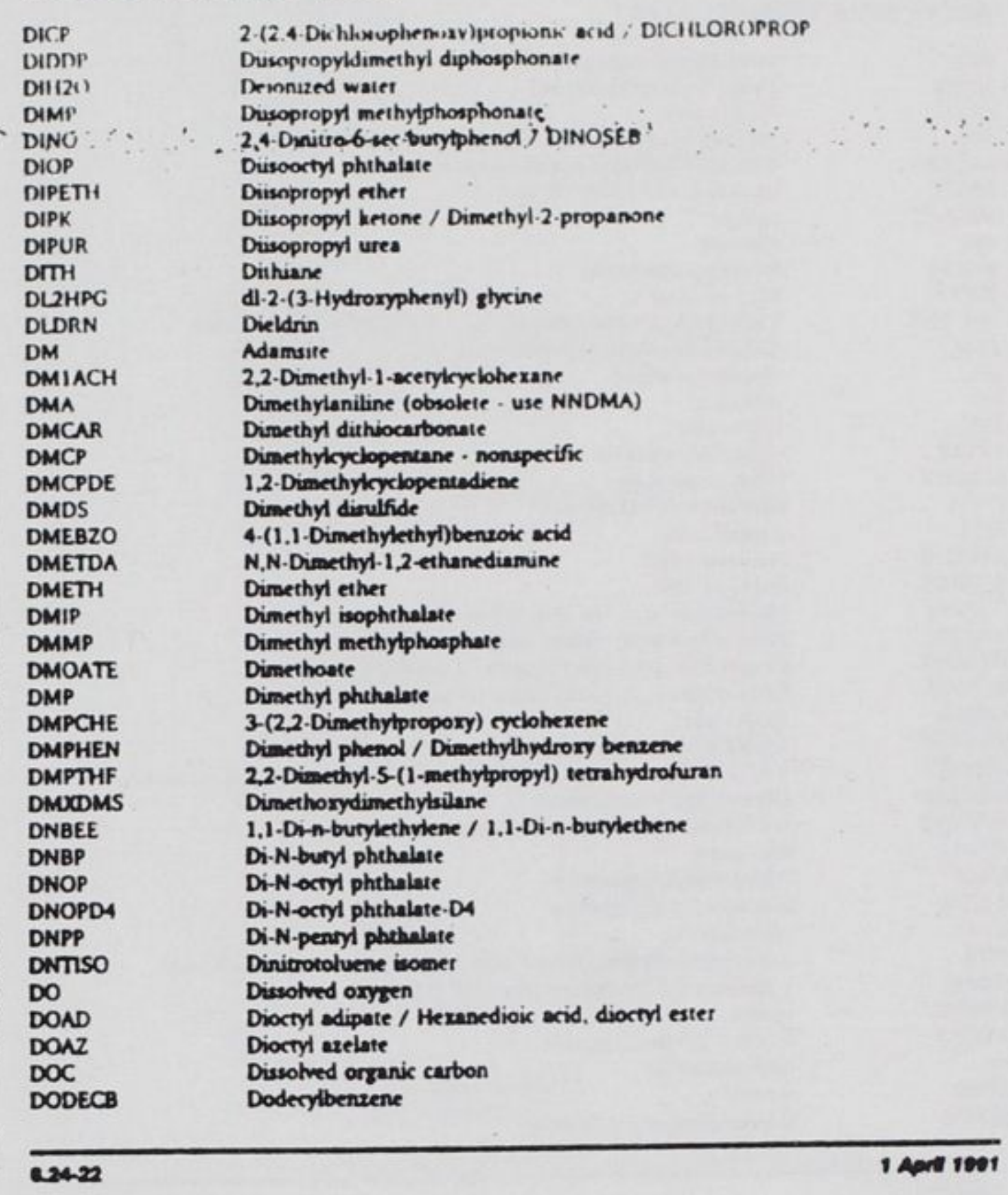

Toet Namo (Anatyno)

\section{ACCEPTABLE ENTRIES: (Cont.)}

DOETH Dioctyl ether

DPA Diphenylamine

DPETYW $\ldots+$ Diphenyi ether

DPH $\quad$ Diphenylhodraxines - nonspecifix

DPHNY Diphenyl

DPNTLL D.(.).Pantolyl laxtone

DPSULF Diphenyl sullowide

$\begin{array}{ll}\text { DRBM } & \text { Dibromomethane } \\ \text { DSEDIN } & \text { Diseleno dindole }\end{array}$

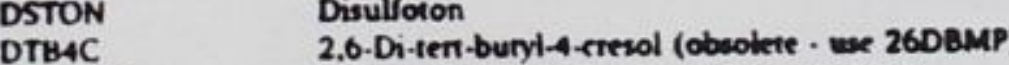

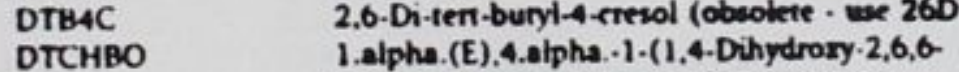

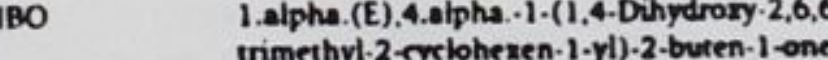

DURS Durban

$\begin{array}{ll}\text { DISCAN } & \text { GC-MS dye xan } \\ \text { EN2192 } & \text { S-2.Dusopropylaminoethyl methytphosphonic acid }\end{array}$

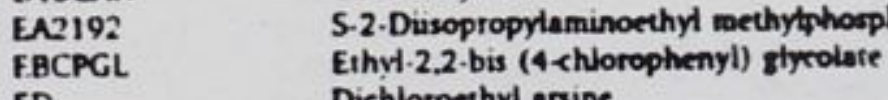

ED

EDBDAS

E.COSL

EMFUR
EMPA

EMPA

ENDRN

ENDRNA

ENDRNK

EPHEN

EFTOX
ESFSO4

ESFSOA
ET3MB2

ETAMBZ
ETBD10

ETBD10
ETCOHS
ETCMUX

ETCVHX

ETIBR
ETHER

Ethyl-2.2-bis (4 echlor
Dichloroethyl ansine

E.Phenylpropanal

1. Eironanol

3-Ethyl-4-methyloctane

Ethyl methytphosphonic acid / Echyl methytphoophonete

Eihyl methanesullonate

Endrin

Endrin oldebyde
Endrin ketone

Ethyl-N.heryl ether

Ethyl phenol / Ethythydroxy benzene

Extraction procedure toxix organic

Endosulfan sullate

1.Ehyl-3-methulbenzene

1.Ehyli-4.methvilber

Ethylbenzene

Ethykseloherane

Btomoethane / Ethyl

14001 1001
2.24

2.24

ACCEPTABLE ENTAIES: (ComL)

$\begin{array}{ll}\text { ETHION } & \text { Ethion } \\ \text { ETHOPR } & \text { Ethoprop }\end{array}$

ETHPOA Ethyl phosphate / Phosphoric ocid, triethyl ester

ETMuCR. $\therefore$ Echyl methesplate

ETOH

EU Europium

F108P Derafluorobiphenyl

Fomaix scid bete-phenyechin ester

FACHXE

FNMPHR Fampher

FARN

$\begin{array}{ll}\text { FATNL } & \text { Fanty alohohols } \\ \text { FC2A } & \text { Fluoroeretic acid }\end{array}$

FE THENE

$\begin{array}{ll}\text { FLRENE } & \text { Fluorene } \\ \text { FNT } & \text { Fenthion }\end{array}$

FORM Formaldehyde / Methyl aldehyde

FREON
FRN112 Freon / Dichloronuoromethane

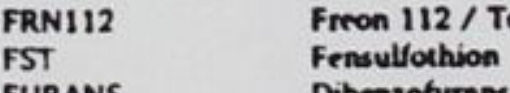

FURANS Dibenzofurans - nonspecifis

GA

GAMMAG

GAMMUS

GB

GBHC

GCLNAN

GD

GRNDY

GUNI

H

H2O

H2S
H3POA
MARD

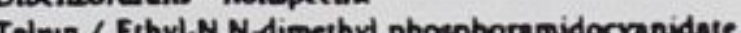

Gallium

Gemans scan / Gamme screen

Serin / lsopropyl methylphosphonofluoride is

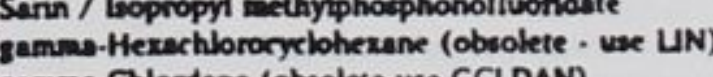

Somen / Pinereatyl methytphosphonof uoridate

Germenium

Green dye

Guanidine nitrate

Levissict

Water

Hydrogen sulfide

Phoophonk acid
Total herdness 
Teet Neme (Analye)

8.24

\section{ACCEPTABle ENTRies. (Cont.)}

HCBD
HCNH

HCNH Herar

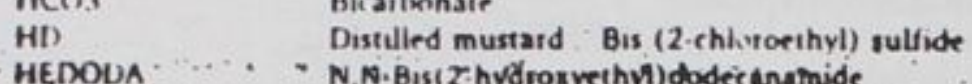

HE,DOUA

HEXANF.

HG

HGEXT
HGTOT

HMTCHF

HMX

HMN
HO
HPC
HPCLE

HPCLE

HPOA

HTH
HWX013

HWX099

HXTB2E

HXADBE

HXADOE

HXcos

HXHMNZ

HXMETA

HXMTSX

HYDRND

HTORZ

HYNB

IGNIT
IMPA

IMPA

INDENE

INDOLE

IPA

ISOPBZ

Hexanoic acid / Coprokt acid

Hexane

Mercury extractable

$26,10.15,19.23$

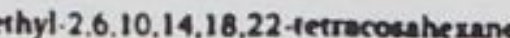

Cycloretramethyleneterranutramine

Heprachlor

Hepiachlor epoxide

Hidrolvasable water

Hypochlonite

Halowax 1013
Halowax 1099

Hexanediok acid. bis (2-ethylhery) enter

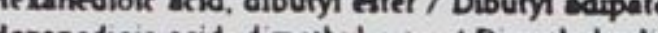

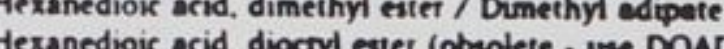

Heracosane

4,5.6.7.8.8A-Hexahydro- AA-mecthyl-2. (1H)-asuleone

1.3.5.7-Tetranzatricyclo(3.3.13.7) decane / Hernamethylene tetramine

Hexamethykyclotrisilorane

IH-Indene, octahydro- / Hydrindane

Hydrazine

7. Hydroxymorbormadien

Ignitabiling

lsopropyl methviphosphonic acis : Isopropyl methviphosphonate

1-Hydroxy-2.3-methviene indan (M.W.140)

indene

Indole / 2.3-Benzopurrole

lsopropylamine

lsopropylbenzene / Cumene

1400|ll 1901
6.24

Toet Name (Analyte)

ACCEPTABle entries: (Cont.)

ISORHR Isophorone

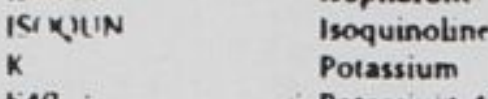

$\mathrm{KB} \quad \mathrm{KO}^{40} \quad$ Polassium 40

KENI Ketoendrin

LA Lewsite

Al40 Lenthanum 140

Lectic acid, gerlic butaneboronete

LAURIC Louric acid

UIN Lindane / gamma Benzenehexachlonide / gemma-Hexachlorocyrbohexane

LPID Lids, perrentsog
Lewisite oride

Bis (2-disopropylaninoethyl) methytphosphonite

MALO Malononitivile

(1)

3-Methy burranoik acid. 3.7-dimethyl-2.4.6-0rtarienyl enter

MBAS Foaming egents / Methylyme blue extive substance

MBOH alpho-Methylbenzyl akohol

alpha-Methylbenzyl accetoeceraie

S-Methylbenzo(C)ecridine

MCPA alpho-Methylbenzyl-2-thloroseretoocetate

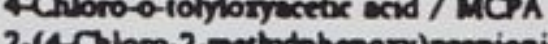

2 Mectid

ME2AEA Dimechyl anenic acid

ME2C11 Dimethylunderanes

ME2HC $\quad$ Dimecthyl mertury

Methyl-2-heptanots

Dimethylnaphetheien

Trimetuylderanes

Trimethyl heranes

Trimethylnephthalenes

Mechyl anonic acid

1.1'Methylenebislpiperidine!

Tolvene-Do

axes

Toet Namo (Analye)

2.24

.24

Toot Mamo (Anatye)

\section{ACCeptable ENTAies: (Cont.)}

MECC6 Methylexeloherane

MECYBU Methylcyclobutane

MECYPE Methylevelodecane

MEFGC :" Methylmeroury

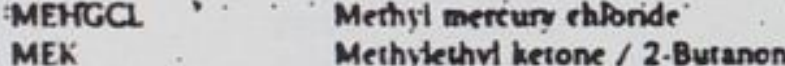

MELAM Melomine/ 13.5 Tremize 2a.6

MEPHEN Methanol

MEPOH 2-Methytpentanol

MERP MeTphor

Methrl sulficte / Thiobismethan

MESTOX Mesinyl oxide i 4-Methyl-3-penten-2-one

METLAP

MEXCLR Mennphos

Magnesium

MIMDRZ Methylhydrazine

MIBCOH Methyl isobunil carbinol (4-methyl-2-pentanol)

MIBK Methrlisoburyl ketone

MIPK

MIREX Mirex

MMIS Methyl methonesullonate

MN Manganere

MNS4 Manganere S4

MNBK Methyl-N.buryl thetome / 2-Hesanon

MO Molvbdenum

MPA Methylphosphonic acid

2.(m-Chlorophenyl)-2.(p-chlorophenyl) -1.1. dichloroecthase

MPK Methylpropyl kerone / 2.Pentanone

MOFIIIO Parathion methyl

MSSCAN Milli-Q.-Filered water

MTRITN Methyl trithion

$\begin{array}{ll}\text { MTRZLL } & \text { Metrazol / Cardiazole } \\ \text { N2KJEL } & \text { Nitrogen by Kjeldohl Method }\end{array}$

N2KUEL NA Nitrogen by

$\begin{array}{ll}\text { NA } & \text { Sodium } \\ \text { NM22 } & \text { Sodium } 22\end{array}$

I apen 1001

\section{ACCEPTABLE ENTAIES: (ComL)}

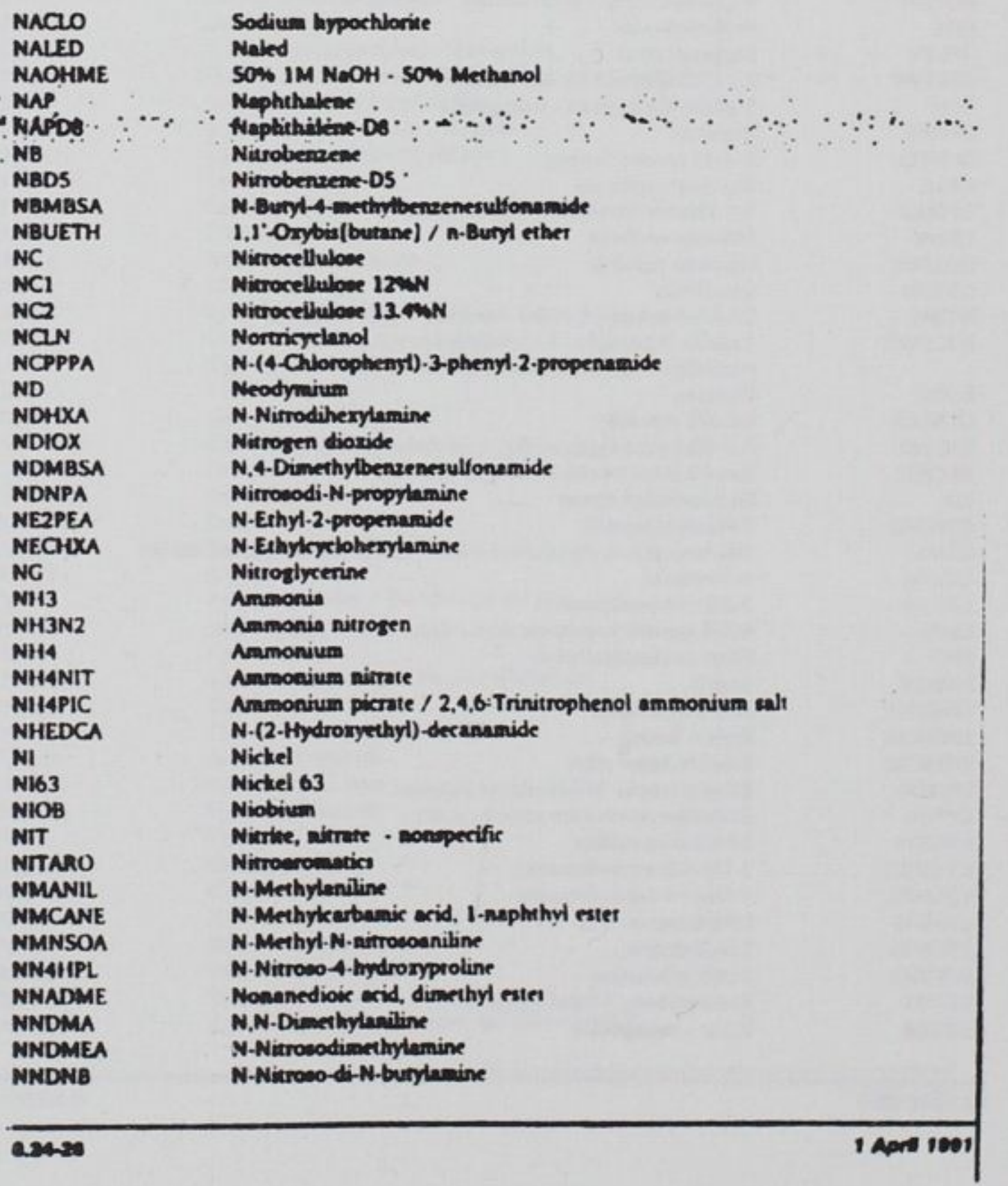


Teat Name (Analyno)

8.24

ACCEPTABLE ENTAIES: (Cont)

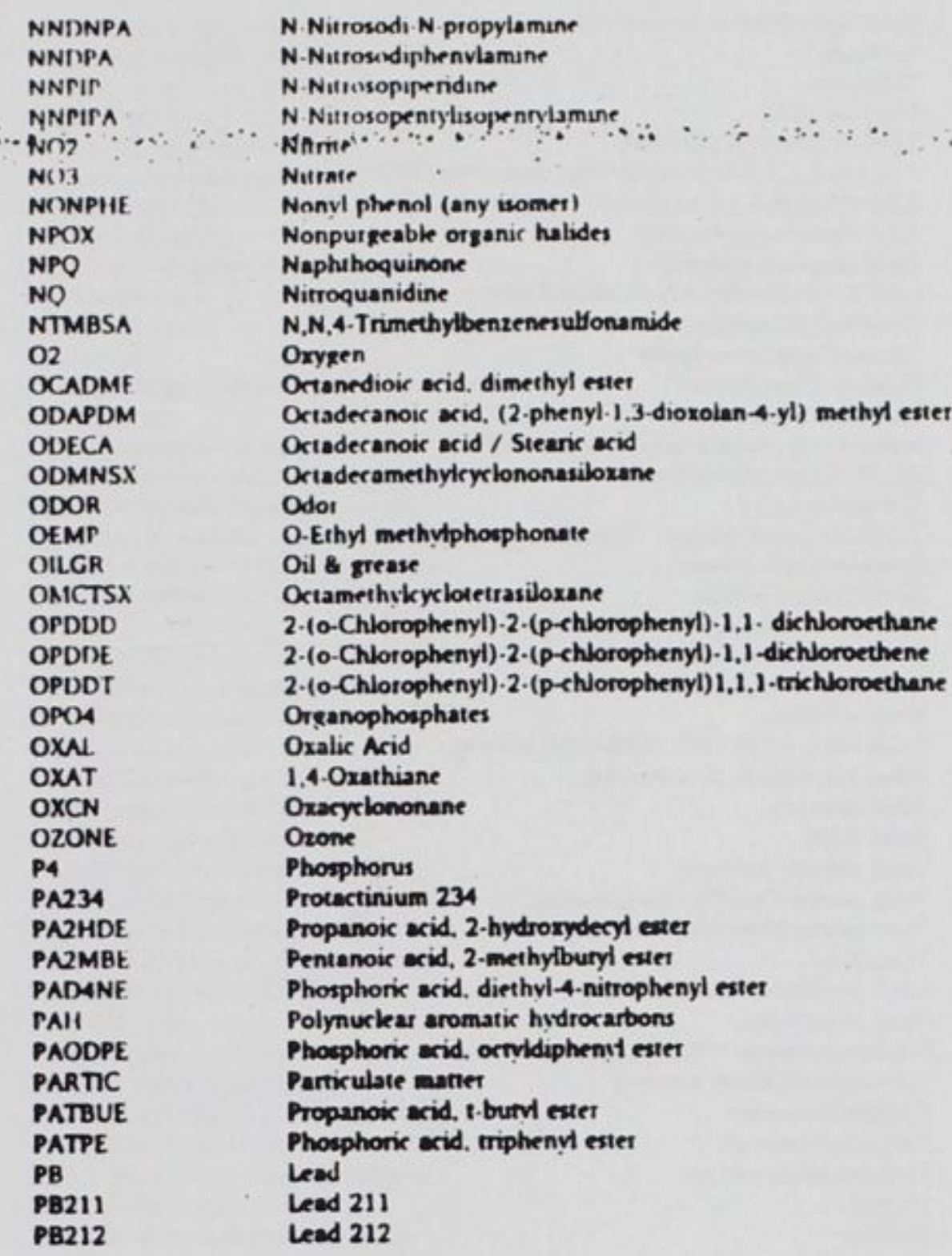

inonteri
Toet Nemo (Anelye)

\section{aCceptable entaies: (Cont.)}

PIPER PIPeridine

$\begin{array}{ll}\text { POA POA } & \text { Phosphate } \\ \text { POAORT Orthophosphere } & \end{array}$

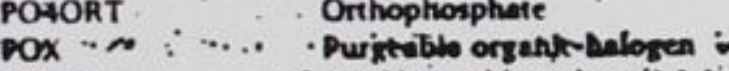

PPDDD 2.2-Bis (p-chlorophenyl)-1.1- dickloroethan

PPDDE 2.2. Bus (p-erlorophenyl)-1.1-dichloroethene

2.2. Bis (p-chlorophenyl)-1.1.1- -mrthloroethens

PRCOHS Propylbenzene

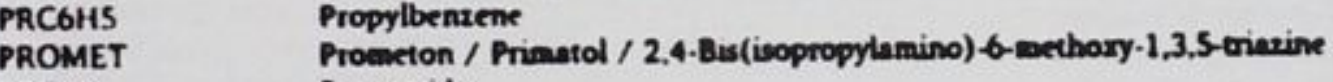

PRONA Pronemide

PROPOX Propitene oxide / Methyl oxianse

$\begin{array}{ll}\text { PRTHN } & \text { Parathon } 238 \text { isotope } \\ \text { P('238 } & \text { Plutonium } 238 \text {. }\end{array}$

PID12

PYR Parene

$\begin{array}{ll}\text { PYRDIO } & \text { PYTene.D10 } \\ \text { PITDDIN } & \text { Pitidine }\end{array}$

$\begin{array}{ll}\text { PYRDIN } & \text { Pitidine } \\ \text { OA } & \text { 2. Dusopropviaminoethvl methylphosphinste }\end{array}$

OA 2 2. Dusopropviaminoethyl methylphosphinste

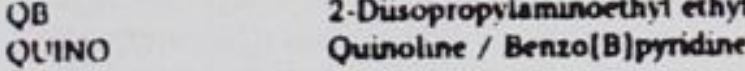

RA Ravium 223

RA:226 Radium 226

$\begin{array}{ll}\mathrm{R} N 228 & \text { Rodium } 228 \\ \mathrm{RDX} & \text { Cyclonite / Herahydro-1.3.5-trinitro-1.3,4-miazine }\end{array}$

RDX Cyclonite
REACT

REDDY Red dye

RESACI Resin acids

RESO Resorrin

RU103 Ruthenium 103

RU106 Ruthenium 100

$\begin{array}{ll}\text { S } & \text { Sulfur } \\ \text { S2C.2 } & \text { Sulfur monochlonide }\end{array}$

SALUNE. Saline

$\begin{array}{ll}\text { SA } & \text { Antimony } \\ \text { SC } & \text { Srandium }\end{array}$

inatit 1001
824

Toet Name (Anaive)

acceptable entries: (Cont)

PA214 Lead 214

$\begin{array}{ll}\text { PHSIY } & \text { Lead snphinat } \\ \text { PCROIT } & \text { PCB 1016 }\end{array}$

PCR221 PCA 1221

PCB292 2 2CB 1292

PCB248 PCB 1248

$\begin{array}{ll}\text { PCB254 } & \text { PCB } 1254 \\ \text { PCB260 } & \text { PCB } 1260 \\ \text { PCE262 } & \text { PCB } 1262\end{array}$

$\begin{array}{ll}\text { PCB260 } & \text { PCB } 1260 \\ \text { PCB262 } & \text { PCB } 1262\end{array}$

Pentachloroherane

PCLORM Dimethyl-2.3.5.6. vichloropicolinic acid / PICLORMM

PCNB Pentachloronitrobenzene

PCYMEN 4.(1-Methylethyl) tolvene / P-Cymene

DD Dichlorophenyl arsine

POMAB

PEGE

PENAMD N.Pentamide

PENTAN Pentane

$\begin{array}{ll}\text { PERTHN } & \text { Penhane } \\ \text { PETOIL } & \text { Pecroleum distillotes }\end{array}$

PETDIL Pecroleum distillotes
PETN

$\begin{array}{ll}\text { PETN } & \text { Penseryihntol iert } \\ \text { PFP } & \text { Peatafluorophenol }\end{array}$

PFP $\quad$ PH

PHAD10 Phenanchrene.D10

PHWVTR Phenenthrene

PHENA Phenecetin

Phenylecetic arid

PHENDS Phenol-DS

PHENLC

HENOL L Phenol

$\begin{array}{ll}\text { PHOR } & \text { Phorate } \\ \text { WTHM } & 1.2 \text {-Benzenedicarbontyic acid / Phihalic arid }\end{array}$

P.:Benzen

Penoryacetic acid

hydroxycyclopentane

1,1:(1,3-Phenylene)echanone
224

.2

Toet Neme (Anelyno)

ACCEPTABLE ENTRIES: (Comt)

$\begin{array}{ll}\text { SCN } & \text { Thiocyanate } \\ \text { SE } & \text { Selenuum } \\ \text { SFOTEP } & \text { Sullocepp / Thiodiphoophoric acid, tectracthyl enter }\end{array}$

SI $\cdots \ldots$ sitica

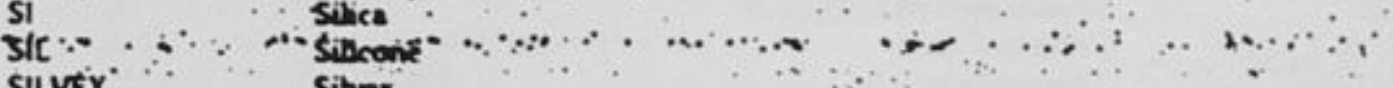

SIN

$\begin{array}{ll}\mathrm{SN} & \text { Tin } \\ \mathrm{SO} 2 & \text { Sulfur Diovide } \\ \mathrm{SO}^{2} & \text { Sulfite } \\ \mathrm{SO} & \text { Sulfate }\end{array}$

(1,5 mans) -7-Oaloro-6-hydroxy-2,4- dimethory-6.-methyl apiro

SQUNL Squalene

SR9O Stontum

$\begin{array}{ll}\text { SSOL } & \text { Sertieable rolid } \\ \text { STB } & \text { SUper tropical bleach } \\ \text { STERO } & \text { Sieroids }\end{array}$

STIGMA Srigmesienel

surophos / Terochlominphos

STMPH Sophnate ion

STMPHA Srpphnic acid (obsolete - 246TNR

STYPHA

SULFID

SUPONA

TI3DCP

TIB2BC
T2DEC
TA

TASTE

TRA

TRP
TCB
TCBI

TCB1

TCB3

Sulluric arid, dimethyl ever

Sulide $/ 2$-Chloro-1-(2,4-dichlorophenyl) mnitdiethyl phosphale

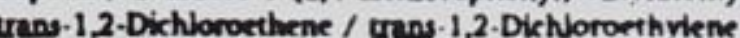

Tans. 1.3. Dichloropropent

une-1-Bromo-2-buryiksclopropan

tons-2-Deerex

Tentalum

Triburylemine

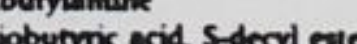

.

Tnburt phosphate

1.,4.5- Tetrichlorobenzen

1.2.3.4. Tetrachlorobenteene

23.3.8-Terechlorodibenzo-p-dioxin / Dioxin 
Teat Neme (Anatye)

\section{acceptable entries (Cont)}

TCDF

TMHOS

TCLAN

TCLiF
TCN
TCOS
TCP

TCOS

TCSAM

TCST

TDCBC

TDGCLA

TDMHS

TOS

TEGLME

TEGLYC

TEMT

TEMP-F

TERO4

TETR

TETRY

TFDCLE
TGLYME

TH

TH227
TH230
Th232

TH232

THCDD

THCDF

THF

THNAP

THPCDD

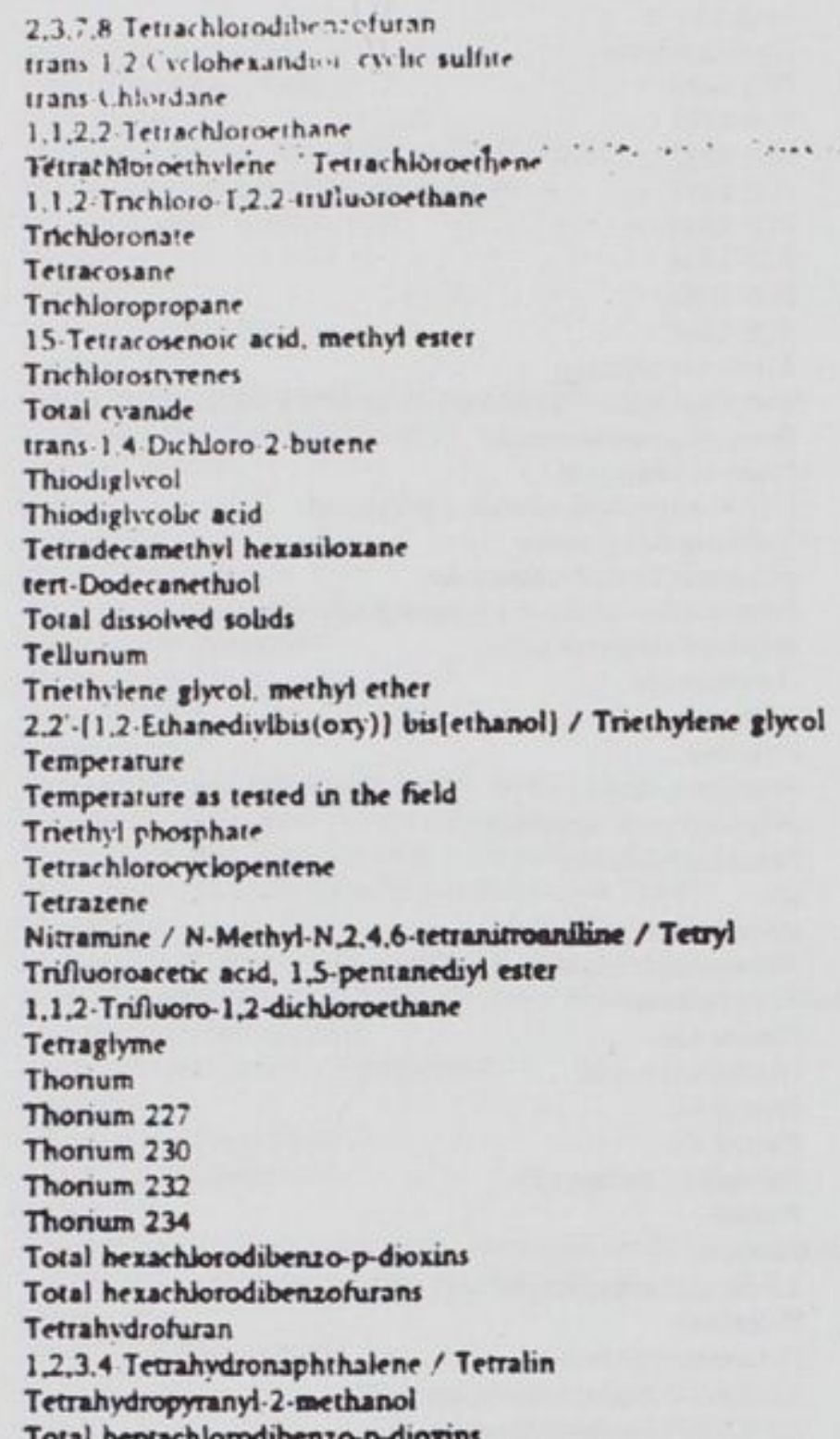

1 Aper reor
B. 24

Test Nome (Anaivte)

ACCEPTABLE ENTRIES (Cont)

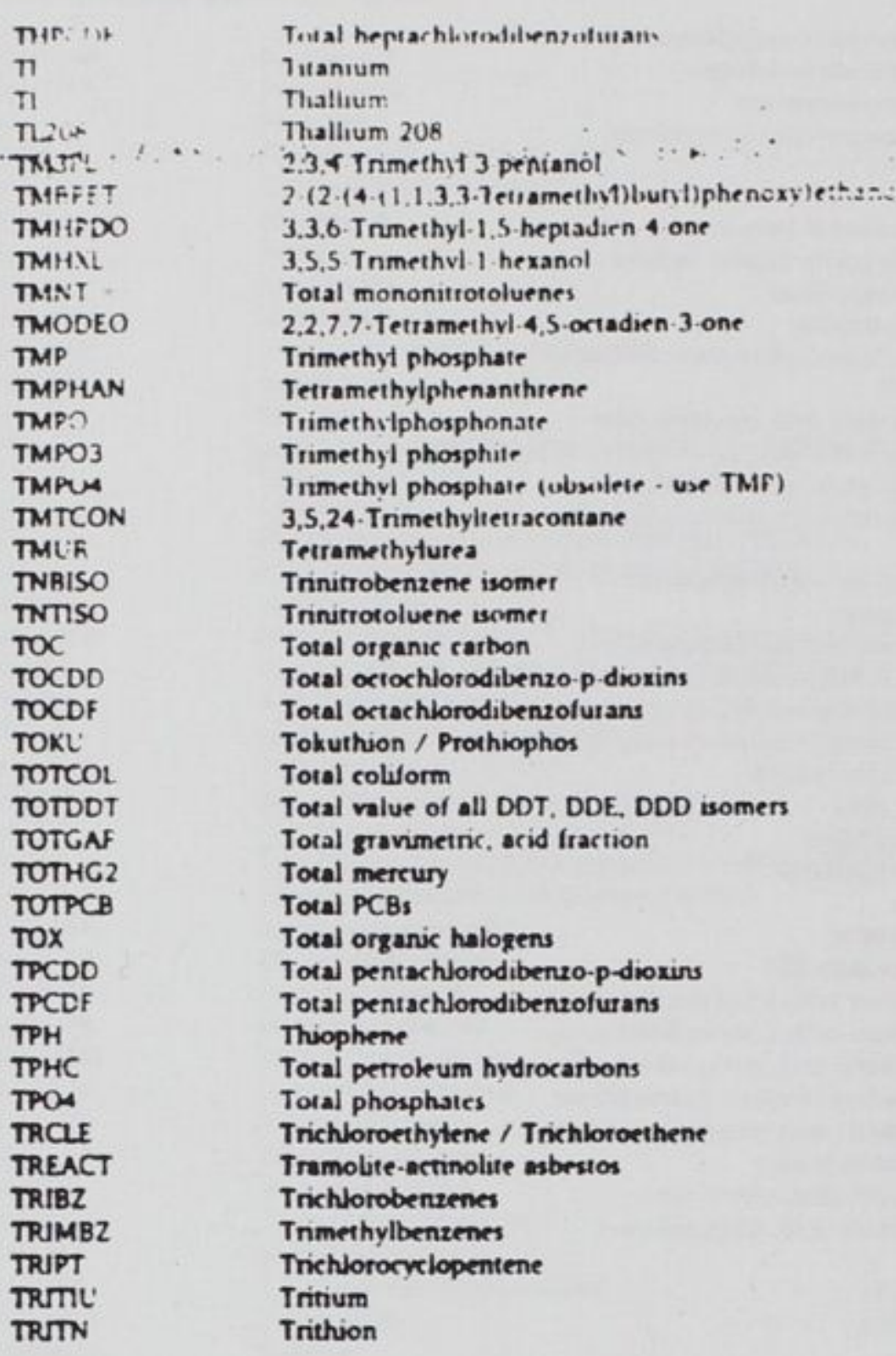

\section{ACCEPTABle ENTRIES: (Comt)}

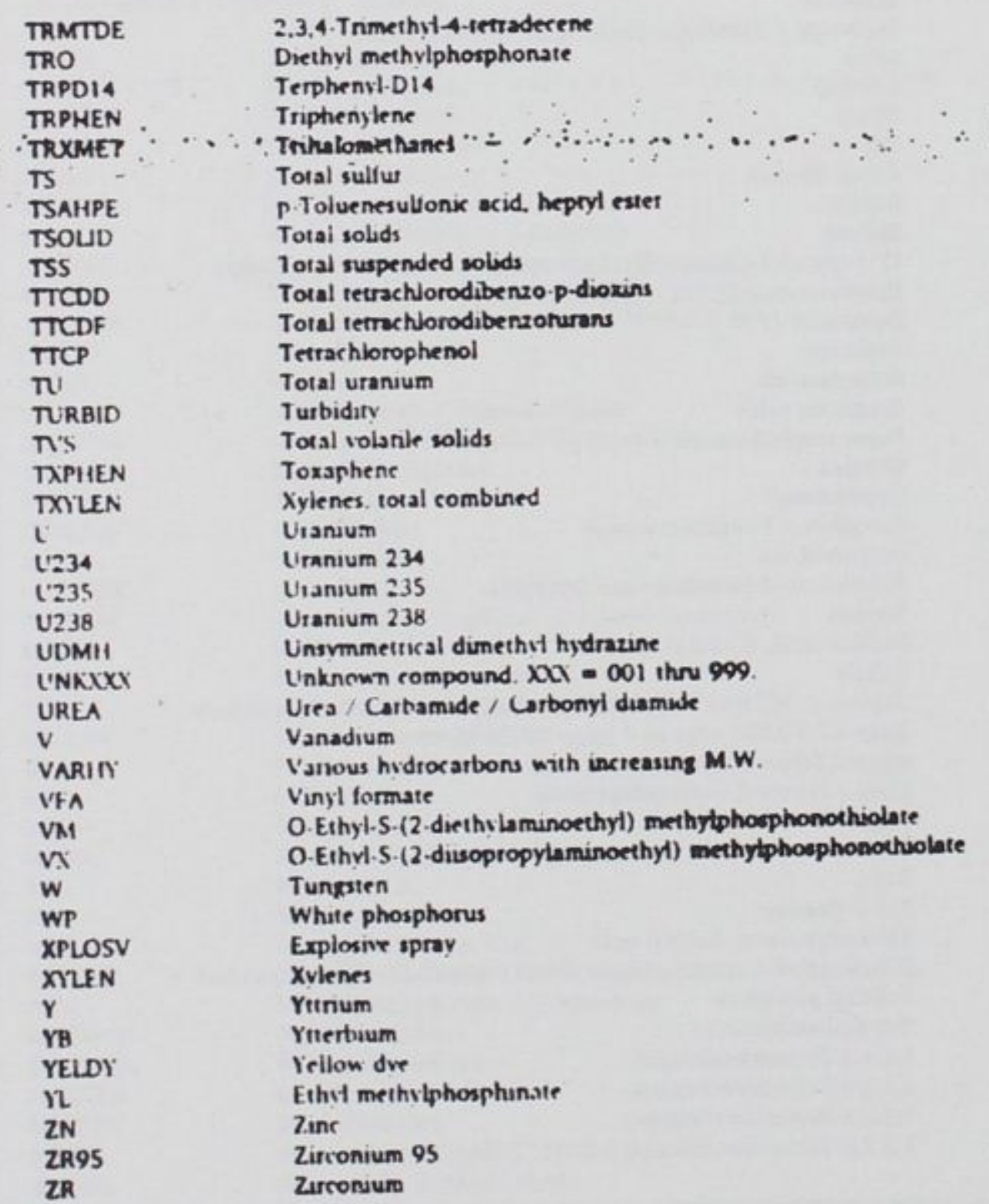

.24

Toet Nemo (Analyto)

ACCEPTABLE ENTRIES: (Cont)

Chomical and Fadiological Date

(Sortod alpheboticelly by Test Nemo)

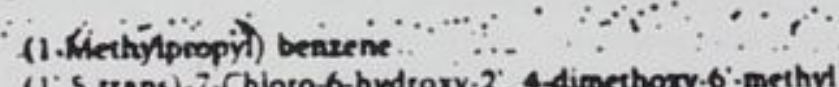

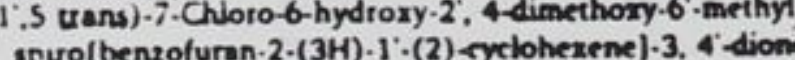

(1.1. Dimethylethys) benzene

(1.3. Dumethytburt) benzen

(2.-Choroethons) ethene

(3bera) - Srigmest-5-en-3-01
$0.1 \mathrm{~N}$ Hydroctuonx acid

1. (2. Butoryethory) ethanol

Aceerl. 3. methyl.5. prenolone

-Acett-4-(1-hydroxv-1-methylecthyl) benzene

1.Benzyl-4. hudrentrenzimidazole

1. Butanol

1.Carbemoyl 3.5-dimethyl.2-pvrazoline

Chloro. 2,4 heradien

Chlorexane

1.Chooronaphthalen
1. Chlorooctioderane

1.Dodecanol

Eromenul

1. Ethvi.2-methythenzene

Fithyl-2.4 dimethylbenze

1. Eshri-3.methvilenzene

1. Ethulherylbenzene

i. Fthitpropolbenzene

1. Flivoronaphethalene

1. Hieprederanol

1. Hexen-3-ol

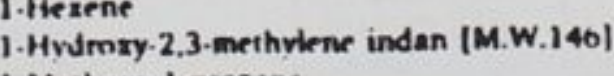

1. Methoxy. 1. propene

1. Methil.7-(1.-methylethyl) naphithalene

1. Methyl-9H--numene

1. Methylbenz (A) anthraven

1.Methykgriopentene

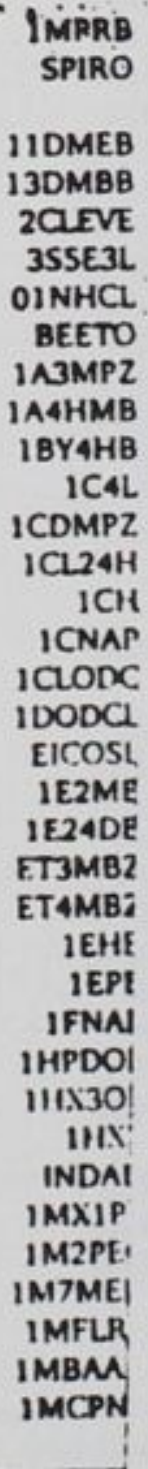

Apent ion 
Toet Name (Analye) 8.24

ACCEPTABle ENTRies: (Cont)

1 Methyldecylbenzene

i Methviethylicurlopropane

1. Methyhindan

1.-Matihyinaphitratete

1. Methyinonylbenzen

1. Methylpyrene

1. Naphithylamine
1. Nitro-2-octanone
1. Nitroheptane

1.-Nitropropene

1. Orcanol

1.PhenVinaphithalene

1. Propen

1. Propenylicuclohexane

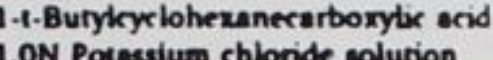

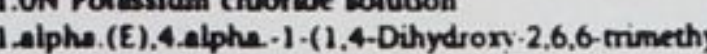

2-pyclohexen-1-y) - 2-buren-1-one

1.1-(1.2. Ethymediyl) bis(benzene

1.1.-Di-n.burylethene

1.1-Dh.n-burylethylene

1.1.Dichloroethane

1.1-Dichtoroethene

1.1-Dichloroethylene

1.1-Dimecthykrelopentan

1.1-Diphenylhydracine

1,1-Oxybis (2 ethoxy

1.1. Thiobis (benzene)

1,1:(1.3.-Propanediyl) bisfbenzene]

1,1-Methyliknebis[piperidine]

1.1-Ombislbutane]

1.1.1.-Tnchloroethane

1.1.1.2. Tetractloroethane
1.1.2. Tnichloro-1.2.2-erifuoroethane

1,1.2-Iniluoro-1,2-dichloroethan

1,1.2,2-Tetrachloroethene

1,1,2,2-Tetranethykyclopropane

140ent 1001

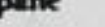

1 MIPB

MFC.PR

IMEINI)
IIMNNA
IMNB
IMPNB

MPYR

INAPA

INHP

INPNOL
IOCTOL

IPNAP

ICIL.
IPECHX
ITECHA

ITBCHA

DTCHBO

DPETYN

DNBEE

$11 D C E$
IDDCE

$11 D C E$
$11 M C P E$

$11 M C P E$
$11 D P H$

EGMEE

DPSULF

$13 D P P R$
MEBPIP

NBLETH

11 TCE

TRCLEA

TPDCE

TCEA

2047

Toet Nemo (Anelye) . .24

aCceptable entries: (Coml)

1.3-Dinworobenzene

1.3. Dimethylbenzene

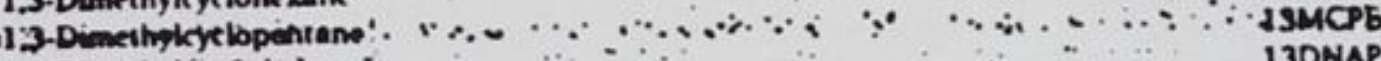

1.3. Dimbithytriaphithalene

1.3-Dinittobenzene

1.3-Diphenvipropane

1.3.5. Tnimethylbenzene

1.3.5-Tnmethvikerlohe

1.3.5. Trinitrobenzene
1.3.5.7. Tetrazatrinitio| 3.3.13.7/decane

1.4. Diacervilienzene

1.4. Dichlorobenzene
1.4. Dichlorobenzene D

1.4. Dichlorobenzene Dor
1.4. Dichlorobulane

1.4. Dinuorobenzen

1.4. Dihidro. 1.4-methanonaphthalene

1.4. Dimethoxianthrarene

1.4. Dimethittenzene

1.4. Dimethvikyclohera

1.4. Dinitubenzene

1.4. Dioxane

1.4. Hersdiene

i. - Bis (1.1-dimethylethyl) -3.3. dimethylbicyclo (3.1.0) berane-2-one

i.5-Dimethytnaphthalene

1.6-Dimethylindan

1.0-Drmethyinaphthalene

1,0,7. Tnimethyinaphthaiene

1.8-Dimethylraphithaie

IH-Benzotnazole

2-(1-Methvitethyl) nephthalene

2-12.4. Dichlorophenoxy) propionic acid

2. (2.4.5. Trichlorophenoxv) propionk exid

2-(2.) (4-(1,1.3.3. Tetramethyl) bur

2.(2-Cyanoethyl) cychoheranon
2.(2- Methoryethoxy) ethanol

1apell 1001
8.24

Toet Name (Anaive)

ACCeptable entries (Cont)

1.1.3 Tnmeihylky toherane

2 Henjenedicarbontir erv

1.2 Dibromoethane

1.2. Dichlorobonenzene id

1.2. Dechboroethane

2. Dichloroethenes

2-Dichboroethylenes (cis and trans isomen)

2.Dichloropropane

1.2. Dumethylkyelopentadien

1.2. Dumethylknelopentane

2. Diphenvibensene

1.2. Diphenylhydrazin

1.2. Eporycycloberene

1.2. Eposyechylbensene

1.2.3. Benzorinazote

1.2.3. Trichloropropane

1.2.3. Trimethylbenzene

1.2.3. Trimethylcyrlohe xane

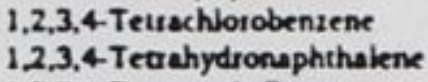

2.,3.4. Teramethy

1.2.3.4.5-Pentuhydromeyclopentane

$1,2,3$, S. Terrechlorobenzene

1.2.4 Trichlorobenzene

1.2.4 Trimethylbenzene

1.2, .4. - Tetrachlorobensen

1.3. Benzenediol

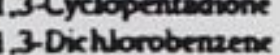

1.3-Dichlorobenzene- DA

1,3.-Dichboropropene

1.3. Dichloroprope

$\cos 2$

$113 \mathrm{MCH}$

Piti

IOEBRE
IEDCLO

TEDCLO

$120 \mathrm{CLC}$

$2 \mathrm{DC}$

12DCE

12DMB

DMCPOE

12DNAP

$12 \mathrm{DPB}$
$12 \mathrm{DPPH}$
$12 \mathrm{EPCH}$
$125 \mathrm{~S}$

$12 E P E B$

23TCB

$23 C P R$

$123 \mathrm{TMB}$
$23 \mathrm{MCH}$

TCB

THNN

$10018 \mathrm{D}$

PHYCP
TCB
$124 T C B$
$124 T M B$

$124 \mathrm{TMB}$

24MCH

RESO

$13 \mathrm{CPDO}$

$130 C 18$
$13 D B D 4$

$130 \mathrm{CO}$

13DEB

1Apert 1001

.24

Toet Namo (Anatye)

ACCEPTABLE ENTRIES: (Comt)

2. (2-N.Butoryecthory) ertanol
2. (2.-Phenorvethoxy) ethenol

2. (2. Phenoryethoxy) ecthanol
2.- (4) Choro-2-methylphenory) propionic acid

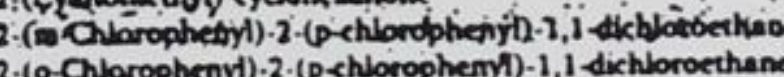

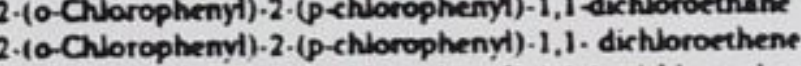

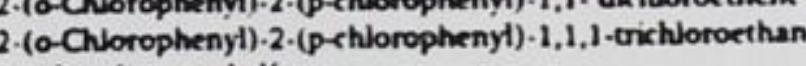

2.(1-bumi) - A.methylura

2. Amino-4 nutrotolvene

2. Amino- 6 - -dinitrotolvene

2. Broma-1 ethoropropane

2.Butanome

2-Butene

2. Butomethanot phosphate

2. Bunyl-1 - oxtanol

2. Bund.N-methylnotleurine, methyl ene

2-Burythetrahydrofuran

2-Chloro-1. (2.4-dichlorophenyl) vinyldiechyl phosphate

2. Chorot-methon

2. Chlorobiphent
2. Chloroerhutunnl e ther

2-Choronsphithalen

2. Chlorophenol

2.Chlorophenol-D

2. Chlorotoluene

2.Chorom

2.-Cresol 2 -Cycheren-1-ol

-Crelohent-4.6-dinutrophenol

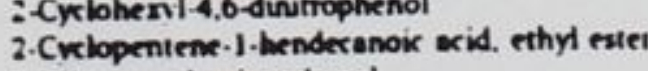

2. Disoprophaminorthanel

2. Dusopropplominoethanyl ethyl methytphosphonate

2.Disoproprlomin

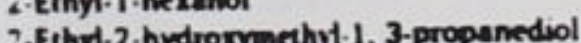

2.Ethyl-4-methyl.1.pentunol

$+\infty$

2BEETO

2PXEX

MCHN

TiTOOO

OPDDD

OPDDE

$460 \mathrm{~A}$

AMGOT

BRHXA

MEK

2BUXEL

B100L

SBNMNM

SUPONA

C6MPZ

2CLPP

CNAP

2 2CIPOA

avis

2MP
2CHEIL
2CHE 10

CHASD

KB

OB

EEIHXI

2EIHXI

IAnent 1891 
ACCEPTABle ENTAies: (Cont.)

2 Fithurwinlolutanol

2 Ethvilhe sano

2. Puorobuphenyl

2-Fluorophenthol

2. Hendecanol

2. Heplanon

2. Hydrontrenzaldehyde

2.Hydroxybiphenyl

2. Hudrombutanedioic acid, dimethyl ester

2- - Isoburynic anid

2-Methory-1-propene

2-Methorv-2.3.3-rnimethylburane

2-Methory -3.6 -dichlorobenzoic acid

2.Methyl. -1-doderanol

2.Methyl-2.-butenediamide

2. Methyl-2. hydroxy-3-bury

2-Methyl.2-propenol

(1,2-ethanediyl eater

2.Methyl-3-herene

2.Methyl-3-pentanone

2.Methyl-4-(1-buryl) phenol

2-Methyl 4 - chlorophenol

Methyit 4,6-6initrophenol

2-Methyl. s-chlorophenol

2-Methyl-a-(1-burnyl) phenol

2-Methylbenzyl akohol

2-Methylbutane

2.Methyleyclopentanone

2-Methylyctopentanol

2.Methylderane

2-Methyldodecane

2-Methylhepecane

2-Methylherane

IAnt 1901

-2-ycloheren-1-one

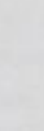

$2 M \mathrm{MON}$
$2 \mathrm{MDO}$
$\mathrm{MDCL}$

2 MDOD

$2 \mathrm{MCO}$

.2041

Toet Namo (Anolye)

.24

ACCEPTABLE ENTRIES: (Comt)

2.2.Dimethyl-5-(1-methylpropy) tetrahydrofuran

2.2. Dimethylbutane

2.2. Oxybisiethanol]

2.2. 3.3 .4 .4 .5 .5 - Octachlorobiphenif

2.2. 3..4.4. 5.6-Hepenachlorobipheny

2. 3.4.5.5. Her.

22.3.4.5.5.6. Heplachlorobiphen

2.2.5. Tninhlorobiphenvid

2.2. S.S. Teurachlorobipheny

2.2.3.3. Terramethytpentane

2.2.4. Trumethyl-1.3. pentanediol 1 inden

2.2.4.4.7.7. Hexamethy

2.2.7.7. Tetramethvi-4. S-oxtadien - 3-one

2.3. Benzopintole

2.3. Drhloro-1-prop

2.3. Dichloophenol

2.3. Dihidrobenzofuran

2.3-Dimenthylnaphthalen

2.3-Dimethvibutane

2.3. Dumethylpentane

2.3. Dimethylphenol

2.3.4-Trimethyl.3-pentanol

2.3.4. Trmethyl-4-terraderene

2.3.4.5. Tetrachlorobiphenst

2.3.4.6-Terrachlorophen

2.3.5. -Trichlorophenol

2,3.5.6. Tetrachlora- 1,4-benzenecartionylic acid dimerthyl eater

2.3.5.6. Tetrachlorophenol

2.3.6. Trimethylopphthalene

2,3.7-Trimethylortane

2.3.7.8. Tetrachlorodibenzofuran

2,4-Bia(tisopropylamino)-6-methoxy-1.3.5-tiazine

4-Dichlorophenol

14001 1001

-
DMPTHF

DECLY

$250 C C B$

26HPCB

SHPCB

$245 P C B$

$225 \mathrm{SCB}$

$24 T 13 P$

$247 \mathrm{HOI}$

$226 \mathrm{TMO}$

MODEO

INDOLE

$230 \mathrm{DP}$

COUMRN

23DNAP

2302 $23 \mathrm{DMC}$

23 DMC4
$23 D M C S$

23DMP

TRMTDE

2345CB

$2346 C \mathrm{CP}$

235TCP

DCPA

DCPA
$2356 C$ P

236 Tnin

23 Tak)

TCD

PROMET

$24 D B$
$240 C 1 P$
2.2019 acceptable entries: (Cont)

2 Merhutnaphithalene

2. Methilioriaderan

2-Methylpentanol

2. Methylpropane

C... MEPOH

$2 M C 3$
$2 M P T$

2MPAHT
2MPAME

2.Methyltertroder

2.Methylietrahydrofuran

2-Methylthio-4hydroxypyrimidine

2-Methylunderanal

2. Naphthylamine

2-Nitro-m-eresol

2-Nirroenitive

2.Nucroeniline

2-Nitrophenol

2-Nitropropan

2. Nirrotolvene

2-Nomedecanon

2.Phentanone

2.Phenylethanol

2. Hicoline

2.Propenol

2-eer-Buryl-4,6-dinitrophenol

2. ON Potassium chloride solution

(DOT reloted)

22. Bis (methylmersapo) propane

2.2-2-Bin(p-chlorophenyl)-1.1-dichloroecthene

22. Ais (p-chloropheny))-1,1-2ichloroethene

22. Bis(p-chloropheny) - 1,1,1-trichloroer hane

2.2-2.Dis(p-chlorophenyi)-2-phen

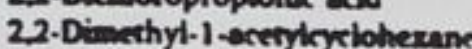

2.2. Disethyl-1-propenol

2MTPY

2MTH

MDCL
$2 N A P A$
$2 N 3 C$

$2 \mathrm{~N} 3 \mathrm{C}$

NANIL

NBZL

2NPN

2 NODCO

MPK
2 PHXEL

2PHXEL

2PETOH

2PICO

258460

$2 \mathrm{NKCC}$

BCPHCE

2BEMDE

2BMMPA

PPDDD

PPDOT

DANA

DMIACH

arese

1Aperd 1981

24

Toet Name (Anetyro)

ACCEPTAOLE ENTRIES: (Comt)

2.4-Dichlorophenoryaretic arid

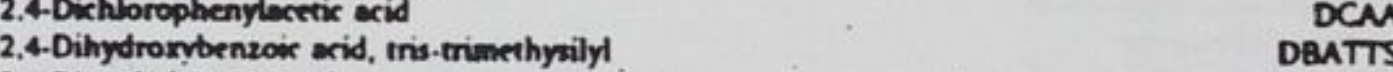

29-Dinequbl-2.pentanol

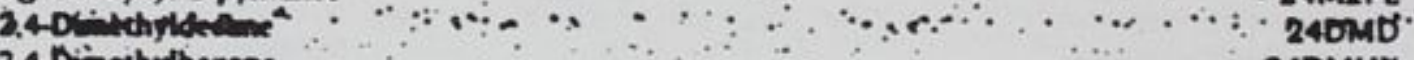

2.4. Dismethylpentene

2.4. Dinitro- 6-ser-buntiphenol

2.4-Dinitrophent

2.4-Dinitroroluene

2.4.-Oichlorobipheny

2,4.5-Trictlorophenol

2.4.5. Tnich orophenoryaretic arid

2.4.6. Tribromopheno!

2.4.6-Trichlorophenel

2.4.6. Trimethvioctane

2.4.6- Trimethitpyidine

2.4.6. Trinitrobenzaldehy

2.4.0. Trinitrophenol ammonium ralt

2.4. - Trinitrmesomeind

2.4.6-Tninitsuoluen

2.5-Cycloheradien-1.4-dion

2.5-Dichlorophenol

2.5-Diethythetrahydroturan

2.5-Dimethylphenenthren

2.5. Dimethytphenol

5.

2.6-Di-ter - burnl 4 - methylphen

2.6-Diter buryl seresol

2.6-Dirhlorophenol

2.0-Dimethylortane

2.0.Dimethitphenol

2,0-Dimethrtayrene

2.6. Dimethylender

axsen

$24 D M C S$

24DMPN

24DNP

24NPDP

$240 \mathrm{DCB}$

2457

$246 \mathrm{TC}$

$246 \mathrm{MMO}$

$246 \mathrm{MPY}$

ATNBA

$246 \mathrm{TN}$

NHAPIC

$246 \mathrm{TN}$

$246 \pi \mathrm{N}$

$247 \pi M$

$250 \mathrm{P}$

$25 \mathrm{ETH}$

2SDMPA

2SDMP

250THF

26DBMP

$2600 \mathrm{MP}$

$200 M$

26DMP

26DMST

26DNA

14 art 1901 
ACCEPtable Entaies: (Cont.)

2.6 Dinitrotoluere

2.6.10.14 Tet1amethviheptadecane

2.6. 0.15:19.23-Heramethyl-2.6.10.14,18.22- -erracosphexane

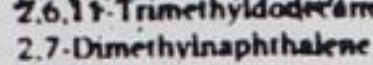

2.7. Dimethvinaphither

2.7. Dimethylortane

2.10. Dimethylundecene

3. (1-Methylethyl)-1H-2.1,-3-benzothiadiazin -4(3H)-one-2,2-dioxide

3. (2.2. Dimethylpropoxy) cyclohexene

3.(Chloromethyl) (recioherene

3-(1-Buryl)-pentane

3.Amino-2.5-dichlorobenzoic acid

3.Butenylpenryl ethet

3.Chloro 1-propene

3.Chlorophenol

3.Chiorot

3.Cyclohexyldecane

3.Ethyl.2.2-dimethylpentane

3-Ethyl-2.5-dimethyl-3-herene

3-Echyl-4-methyl-2.5-furandione

3.Ethylphenol

3-Ethylphenol

3-Hvdroxv-2.7-dimethyl-4. (3H)-pteridinone

3-Hydroxybenualdehyde

3.Methory -2-evelopenten-1-on

3. Methornimidazo

-Mecthoxnotuene

3.Methyl-1-2-onemenol

3-Methyl-2-herenol

3-Methyl-2-nirropheno

3.Methyl-2-pentene

3-Methyl-5-propytronan

1Aporl 1001

2.24

Teet Neme (Anetryo)

ACCEPTABLE ENTRIES: (Com)

3.5.5. Trimethyl-2-exclohexen 1 -one

3.6-Dichloronuoren.9-one

3.7. Dimethylnonane

2. D. Dementhylundecale: :

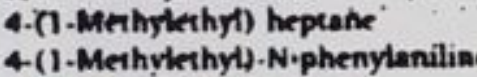

4.(1-Methylethyl). N. phenyld

4.(1.-Methitethyl) toluene

4.(2-Aminoethyl) pyrocatecthol

4-(2.4-Dichlorophenoxy)buryric acil

4.Arenytmorpholine

4. Amino-3.5 dinitrotoluene

4. Aminobiphenvil

4. Bromofluorobenzene
4-Bromophenylphenyl ether
4. Butory. 3.penten-2-one

4 -Chloro. 2 - reesol

4.Chloolo-3-reterol

4 -Chloro. 3 methyl

- Chlomen-10lybomaretic arid

4. Chlorvanilure

Chlorophenylphenyl ethe

4.Chlorotoluen

4 Cremol

4.Ethyl-2-ortene

4. Ethyl-2.2.6.6

4. Fluoroaniline

4- Huorodoluene

4. Hydroxy.3.5-dimethorybenusidehwe

4-Hydroxy-4-methyl-2-pentanone

4. Hydroxyazobenzene

4 4. Hydrorvbenzalidehrde

4.-lodomethylquinuclidine

4. Methorycycloher

4-Methyl-1.(1-methylethyl)-biowlo(3.1.0) ben-2.ene

14anl 1001
26DNT

MINTCHE

2711MD

27DMO

$9 D M U D$

$210 D M U$

BTNZON

उCMCH

उTBU

CAMBEN

3BPETY

$3 \mathrm{CICBE}$
$3 \mathrm{CLP}$
$3 \mathrm{CIT}$

$3 \mathrm{MPP}$

3 EHXD

EEBOD

उEP

3 HOCE 20
3HDMPT

उНMB

उM2C1O

$3 \mathrm{MXM}$

3MIPL

3M2CHO

$3 \mathrm{M}_{2 \mathrm{~N} 3 \mathrm{C}}$

$3 \mathrm{M} 2 \mathrm{CSE}$

4 IC3C

and

acceptable EntRifs (Cont)

3 Methyl- e rhinemphrem

7Methylliphentit

3. Mecblikhoianthe?

3. Methylderane

3-Methylherane

3-Methytpentane

3-Methytphenol

3.

3.-Nitroutuene

3. Octanol

3--0xa-3-phenytpropanoik acid, ethyl enter

3.-Phenytpropanol

Phenytpropanoyl chloride

3.Propyttoluene

3.-Dimethylhezane

3-Dimethyipentan

3.3. Dichlorobenzidine

3.3.4.4. Tetrachlorobiphenyl-D6

3,3,6-Tnmethyl-1,5-heptadien $4-$ - on

3.4-Benzoliworanthen

3.-Dihydro-2H-1 - benzopyran

3,4-Dimethyl-1 derene

3.4-Dimecthytphenol

3,4-Epory-3 ethyl-2-butanone

3.4,4-Trumethyl-2. penten

3.4.5.6- Tetramethylphenantivene

3.5-Dimethyl.2-ortoheren-1-ope

3.5-Dimecthyl-3-heranol

3.5. Disecthylphenol

3,5 Dinitrophenol

3.5-Divitrotolvene

3.5.24-Trimethylitetracontas

$2 x$

200

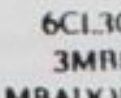

MMOMRY
3MDE:

ACCEPTABLE ENTRIES: (COMt)

4.Methyl-2.pentanol

4-Mecthyl-2.proptl-1.pentanol

4.Methyl.3-penten-2-one

4. Methyl-2y - Auprene

4 Meehylbiphenyl

4.Methyldibenzoth

4-Methylheplane

4.Mechylphenol

4 Mechyppren

4-Nitrophenol

4. Nirropoluene

4-1-Buryl-2-eresol

4.1-Orntphenol

4.4.-Diluorobenzophenoone

4.4. Dimethyl-2.penice

4.4. Dichlorobentophenone

4.5-Dimethyl-2.6-bis (trimethytsilony) pyriaidine

4.5.6.7.8.8. . Hernhydrom

4.6.6- Dinitio-2-2rewal

4.7. Dumethylundecan

S-Chloro-artesol

S-Eihyl-2-methylheptan

S-Eihyl-S-methyldecane

-Methyl-5-hydronyteranoix acid lectoon

5-Mortoren-20l

- propyitrideran

S.7-Dichloro-2-methyllenenzotura

6-Aminohezanoik arid lectean

6-Chloro-3-revel

6.EAhyl-6-methyltulue

a-merhyl-3-implane

MIBCOH MMZPPL MFUR 
Toet Name (Analye)

8.24

acceptable entries (Cont)

O.Methvipunne

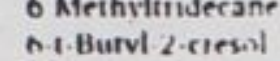

7 Hrdronymorbormadiene

7.12 Dimthylbenz (A) anthriacen

TH. Benz1 DElanthracen-7.

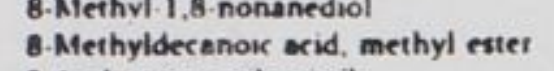

9. Anthresenerarbonimle

9. Aluorenone

9.Methoryanihracene

9-Methylbenzi|Alanthracene

9.10-Dihydro-9.9-dimethylacridine

9 H.Corbazole

OH. Fluoren-9-one

10-Cyclopentulunderanoic acid, methyl

10-Oriaderenois acid, methyl euter

1004 Methanol

17. Methylietrodecanoix acid. methyl ester

14. Methylpentederanic acid methyl

15-Tetracosenoic ecid, methyl ester
16-Methylhepradecanoic acid, methyl ester

16-Methylheptadecan

SOH IM NaOH . SOW Methano

$50 \mathrm{w}$ Hexane - SOM acerone

504 Methylene chloride. SO4 acetone

son Water - $25 \mathrm{~s}$ Methanol - 25w ecetonimite

Areenaphthene

Acenaphthylene

Aretic arid, cycloheryl ester

Aretic acid, ethyl ester

Acetic arid

Anetonitrile

Anetophenone

140014 1001

GMERUR OMTHII)

OTA1 2

PITTRE

$12 D N A$

BDEANT

MMNDL.

NNTRCN

GFLENO

9MXNANT

NTRQU

CARBAZ

9HFLRE

MUNM

10OEME

OMEOH

2MTDM

ЧMPME

MHME

TCSAME

17PTCE

SAOHME

SOHSOA

SOWMAN

NNAPNE

ACNDIO

ANAPYL

MCH.XE

C2AEE

CANET

$\mathrm{ACPHN}$

20010
15.Methylheradecanoic acid, mechyl ester

Toet Name (Analyno)

8.24

acceptable entaies: (Comt)

Ammonium dihydrogen phosphate

Ammonium nitrare

Ammosite nsbesios

- Antilime

Anion elutent

Anthophillie

Anticholinesiesase

Antimnny

Arsenic

Asenic total

Athrsquino

Atsazine

Azsertononane

Azinnhos met
Ranum

Banum
Bentazon

Benzal chlonide

Benzoldehyve

Benzanthio
Renzene

Benzene. D6

Benzenephosphonk: acid

Benzfluoran
Benzidine

Benzidine
Renzo|A A anthracene

BenzolA) phenant

BenzolBinuoranthe

Benzo|B|Alnoorene

BenzolB| Inophthol 1.2. D) thiophen

BenzolBinaphitho|

BenzolBlithiophene

BenzolB| Briphenvilene

Renzo/B|triphenvitene

Aenzo(E)pyrene

Benzo|G,II.IJ nuoranthene

1A0nt 1001
8.24

Toet Name (Anolyie)

acceptable entries (Cont)

A inlitis

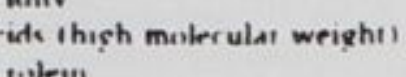

Activerin

Aetiviuim 228

Adamsite

Nkohols (high molecular weight)

Aldrin

Alipheric atrohols

Nipharic hydrocarbons

Alkalinury

Nkalinity - bicatbonate

Nkaliniry - carbonale

Nhanes - hydrox

Nllyw chloride

Allyt ether

alpha, alpha-Dimethylhenzenemethanol

Ipha. alphs-Dimethylphenethylamine

alpha-Benzenehexachlonde

apha-eromoroluen

alpha-chiordane

dipha. Hexachlorocyclohexare

Than-Mechylbenzyl-2-ehloroscetoecetate

alpho-Methylbenxyl acetoscetate

apho-Methylbenzyl alcohol

alpha-Pinene

aphs. Trinitrotoluene

Npha groess.

Apho grous- Lab

Nophe groses-eoluble ecid fraction

Nipha gross-soluble water fraction

Numinumen

Amenable cyanide

Aminoguanidine

Ammonoain nitrogen

2006

A. III)

ACIMIMY
ACRIIN

$A(R Y H$,
$\therefore C 22 B$

DM

ALHMW

ALIC

AI.KAIC

ALKCAR

NLKN

AYIETH

BZAL2M

ABMIC

BTYLAR

ACLDAN

ABHC

MBZCL

$\mathrm{MBZA}$

ALPHPN

246 TNT

ALPGF

ALPGL

ALPGLW

CINAMI

CNAAM

NH3 3
NH3N2

NH4

2.2

Toet Nemo (Anolyto)

ACCEPTABLE ENTRIES: (Com.)

Benzo[G.H.J)pentene

BGHIPY

BZHOUN
BFANT

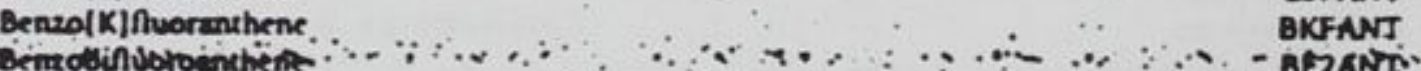

(a)

Bensoir ecid ard

Benzoic acid, methit eser

Benzothizzole

Benzorrichlonde

Benzyl akrohol

Benzyl bromice

Bernilutium ?

beta-Benzenehexachloride

bete. Chiordane

bets. Endosulfan

Gra. Hexachlorocycloherane

Beta gross

Beta gross

Beta grosa. Lat

Beta groses-soluthe acid ftartion

Deta xioss- soluble watet traction

AitC - nonspecifí

Bicyrbo[2.2.1] |hepla-2.5-diene

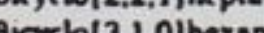

Birvelohent

Binaphthyl

Biolopical oxyzen demand

Bis (2-ehloroetiony) methan

Bis (2-ehloroethyl) ethet

Bis (2-rhloroethyl) suifice

Ris (2 rethylheryll) phithalue

Bs (carbonmethyl) sulfone

Ais (certorymethyl) sulloxide

Bis (elloromethyl) ether

Ras (dimopropylaminoechyl) disulfice

$.24-62$

BENZOA

BZONH4

BT2

BzNic

BZMBR

BZnC

BE7
BBHC

BCIDN

BENSLF

BEGA

BETAG,

BETCL

BETGLA

BETRLW
BHC

HCO3

BCHPD

BCY3HX

BINAP

BOD

B2CLEE

BDD

B2EHP

BCMSO2

BCLME

DINDS

1ant 1091 
Teot Nome (Analye)

.24

ACCEPtable entaies: (Cont)

Bus (dusupropylaminoethyl) methviphosphonate

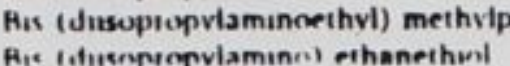

14. (duscpreputamino) ethanol

Ax Lisopropylomimo) ethylsulfide
Bis (disopropulamino) ethylisulionate

Bis (pentanuorophenyl) phenyl phosphine

Bis (tinmerthydalyl) ondis acid

Bismuth

Bismuth 212

Bladex

Bolsıat

Boron
Bıomacil

Bromide

Bromobenzene

Bromochloromethane
Bromodichloromethane

Bromoeithane

Bromolorm

Bromome

Butanedior arid. dimethyl ester

Butanor acid, I-hexyl ester

Buryilbenzene

Butylbenzyl phihala

Burblethyi ether

Buydmethyl phithalaie

Buryiphth
C17 olkan

C18 alkane

C1811300 Unknown

C2211400 Unknow

CB slkane

Codmium

Calcium

Cakculated hardness

Camphor

Caproic arid

1A0011 1001

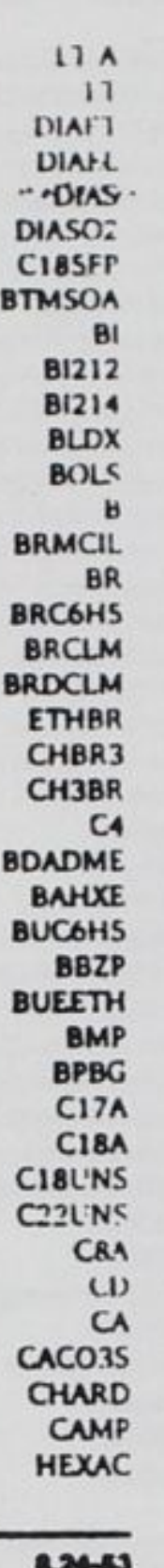

2006

0.24

Tost Nome (Anoive)

ACCEPTABLE ENTRIES (Cont

wpiolariam

Gathamir arw

watbon dionise

certion disultitide

Carbon monoside

Carbon ietrathing

Garbonic acid, dimethyt este

Garbonyl chlonde

Cartonyl dismude

Cardiazole

Esiechol

atton exchange caparity

Cenum 141

Cenum 144

Cesium

Cesium 134

Cesium 137

Chemical orreen demand
HLORAMBER

CHLORAM
Chlorate

Chlordene

Chibrdene

Chloride

Chlonnated benzenes

Chlonnated naphthalen

Chlorine

Chiorine demand

Chlorascetic acid

Chloroscetopheno

Chorobenzene

Chiorobenzene DS

Chlorocyctoherane

Chlorodinitrobenzene isome

Chloroethane

Chlorofluoromethan

.24-4

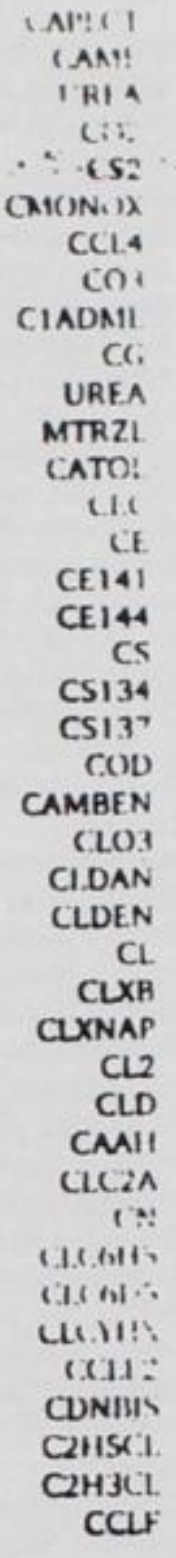

IApent 1091

Toet Neme (Analyno)

8.24

aCceptable entrues: (Cont.)

Chloroform

Chlorolorm.D

Chlotomethane
Chloromethyl methis ether

Chloronemphita

Chloropyntos

Chlorotoluen

Cholestan

Chromate

Chrmene

Chrmene D12

Chnsotite asturstus

cis 1 Biomn-2 chlororvelohexan

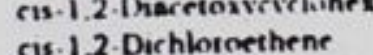

cis-1.2. Dishlorethilene

cis-1.3-Dwhknopiopene

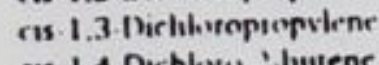

cis 1.4 Duhbown:

eis Chlordane

Contati

Contalit in:

Colot

Copper extractatio

Copper total

Cimesiuniry (tendencr to conode)

Conumaphos

Coumara

Grocublolite astersius

Gyonflex

Cumene

Cranide

Cyanide. fiec foim

Cyanogen chlorition
Cyrlododerane

ingerd 1001
8.2

Toet Nemo (Anebre)

ACCEPTABLE ENTRIES: (Comt)

Cycloherane

Creloherenone

Cyclobenene
- Eyctionzenene oride

Cueloherytbenzene

Cyrbootatertsene
Crelopentaderene

Crelopentanecartoraldehyde

Cyclopentanone

Cveboetramethrleneterranitramine

D. (.). Pantoly lectone

Derthal
Delepon

DCM

Decanluorobiphenvl

Deramethyleve lopentasilorane

Derane

Derylbenzene

Della-Rentenenerecthloride

rocrelohesane

Demeton-U

Di-N.butyl phithalate

Di.N-ortyl phthalate

Di N-ortyl phithalate. Dr

Di-N-pentyl phthals

Diacetome akothol

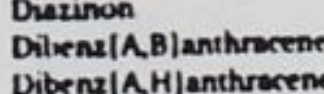

Dibenz|A lecridine

Dibenzofuran

Dibenzofurans - nonspecific

Dibenzothuophene

Dibsomorchloromethene

Dibromorthoropiopane

2.24
CYHX CHOOH

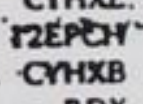

RDX

CYPD

CPOWL

CYPNE

HMX
DPNTIU

DCPA

DCAM

FIOBP

DCMPSX

DECIB

DIH2O

$\mathrm{DBHC}$

DEMO

DEMS

DNBP

DNOP
DNOPDA
DNPP

DINCNI

DUNZ
DBABA

DBaiA

DBNA

DBZFLR

DBZTII

DBRCLM

DBC.
DERDCM

1 Aodel 1001 
aCCEPTABle ENTRIES (Cont.)

Dihromomethane
Diturvi adipate
Diturut hlutendaie

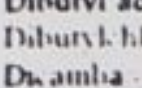

Dichloroseretohimite-

Dirlikotulenzene- mumspecilin

Dichlorobenzenes

Dichlorotenzophenone
Dichlorobiphenyls

Dichlorodifuuoromethan

Dichboroethyl arrine

Dichlorofluorometh

Dichlorolormoxime

Dichloronaphthalenes

Dichlorophenlactic

Dichlorophenyl an

DICHLOROPROP

Dicurlohexyit phithalase

Dirvirlopentadiene
Gereldrin

Derthanolan

Diethyl ether

Drethyl methwiphosphonate

Diethyl phithalate

Diethyl phthalote

Diethylaming

(Diphosphonate

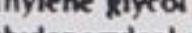

Diethylenesycol monomethyl ether

Oussoburyl phthalate
Disooryd phthalate

Disopropyl ether

Disopropyl methytphosphonate

Disopropyl methy

Disopropyldinethyl diphosphons

Dimethoate

Dimethoxydimethylsilane

Dimethyl adipote

10001

Toet Nemo (Anatyo)

8.24

ACCEPTABLE ENTRIES: (Cont.)

Endosulfan I

Endosulfan II

Endost

Endritin aldehyobe

Endrin ketone

Ethanol

Ethanolamine

Ethei

Ethion

Ethyl-2.2-bis (4-chlorophenvi) givolate

thyl aretale

Ethyl bromide

Ethvil methacrviate

thyl methanesulionate

Ehyl methilphosphinate

Ethyl methviphosphonic acid

Ethvil phenol

Ethyl phosplate
Ethyl. N-hexvi ethet

Ethvi-N.N-dimethvil phosphoramidoryanidale

Ethyllenzene

Ethvibenzene.D10

Ethylene glival. monoet hyl ether

Eihylhydrory benzene

Ethylmethyt benzene

Europium

Exphaive spray
Extraction procedule toxic organic

Famphur

Farnesol
Farty akrohols
Fecal coliform

Fensulfothion

Fensthion

Fluoranithen
Fluorene

Fluorene
Fluoride

I Aperll 1001
DBRM

cambs

IXCLB

$\mathrm{DCBPH}$
C $2 \mathrm{BP}$
$\mathrm{CC}$

CCLFF

FREON

$\mathrm{CX}$
$\mathrm{C} 2 \mathrm{CH} 2$

CLNAP

DICLP

DICP

DCPD

DLDRN

DEETH

TRO

DEPDA

DEDMP

DEGLYC

DIBP

DIOP
DIRETII

DIPA

DIPI R

DMOATE

DMXDNIS

20.87

acceptable entaies (Cont)

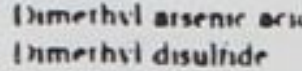

inmerthwl Jithwora

Dimetivl ether

Dimethyl mercurs

Dimethyl phenol

Dimethyl phithalate

Dimethyl-2-propanone

Dimethyl-2.3.5.6-richloropicolinix acid

Dimethylaniline

Drmethwrevelopentane - nonsperitic

Dimethylnaphithalenes

Dimethyipoly sidoxane

Dimethylundecanes

Dinitrotoluene isome

Dioctyl adipate

Diortid ether

Dioxin

Diphenyl

Diphenyl ether

Diphenyl sulfide

Diphenyl sulloxide

Diphenylhydra

- nonspecific

Dissolved organic carto

Dissolved oxyzen

Distiled murtar

Disulloron

A1-2-(3-4)

Dodecene

Doderybenzen

Dopamine

Dursban

2xat

MICA

Mat IH

MESHC

DMM

DIPK

PCLORM

DMC

ME2NA

ME2C11
DNTISO

DNTISO
DINO

DOAD

DOETI

TCDD

DPHNY

DPETH
DPSULF

DPSO

DPA

DSEDIN

1 Aperll 1901

2.24

Teet Nemo (Anelye)

ACCEPTABLE ENTRIES: (Comt)

Fluoraseretic acid

Foaming ogents
Formaldehyde

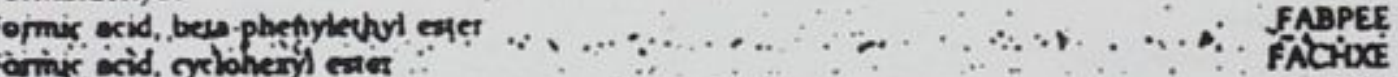

Freon

Freon 112

Gamma-Benseneherechloride

gammo-Chordane

terachlorocyclohexan

Gemma gross

Gamma screen

GC.MS dye kan

Germaniv

Green dye

Guanidine nimrate

Haiowax 1013

Halowax 1099

Henderane

Henerosane
Heprochlor

Hepeachlor eposide

Hepreschlorobiphents

Heplachloronorbomene
Heprechloromorbornediener

Heplachinromorbornedienes

Heproderamer

acid, methyl ester

Heptane

Hepeanoir arid
llesechlornbenum

Herachlorobiphenyts

Hezachlorobutadieme

Hexachlorocyriopent

Herachloroet hane
Herachloronorbomedien

Herschioron

Hezoderam

.2400 


\section{Toet Nomo (Anolyto)}

624

ACCEPTABLE ENTRIES: (Cont)

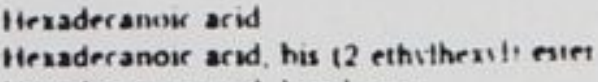

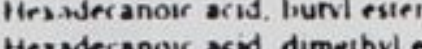

Heradecanoir acid. dimathyl ster.

Herohvdio. 1.3.5. minitro 13.4 . max

Hexamethvitevelotrisidoxane

Hexane

Hexane
Hexanediuk acid. bis (2-ethythexyl) este

Hexanedroir acid. diburyl ester

Hexanediok scrid. dimsethyl est

Hexanow' ach

Hexatnacontane

Hexavaient chromium

Holmium
HPIC. grade water
Hydrazine

Hidrazine

Hidrocarbons (all molerular weights)

Hvdrocinnamyl chloride

Hydroxyanic acid

Hidrozen canide

Hidrolyzable phosphate

Hypochlonite

gnimabiluty

indene

Indeno(1.2.3-C.D)punen

Indole

Iron

lsodrin

Loheptane
tooortane

loopentane

lsophorone

sopropyl methylphosphonate

hopropyl methylphosphons acid

1A001 1001

\section{Teat Namo (Anatyre)}

8.24

\section{ACCEPTABlE ENTRIES: (Cont)}

Methyl isobunt carbinol (4-methyl-2-pentano)

Methyt mercury

Methyl mercun cluderide

Methil oxirame:

Methyl trithion

Methit-N.butyl keton

Methukirirobuitan

Methukycloderan

Methylcyelobexane

Methitwrimpentane

Methrieme tromide

Methitene chlonde. D?

Methvilethsil ketune

Methivethit phenel

Methviethilhudens Ireniene

Methrlhuda azine

Methistirotiunts ketene

Mlethvinaphtilialenes

Methripentyl ketone

Alethiviplenenula

Methylphomphonic acu

Alethitpropist kerione

Methylyne
Mettazol

Millt-O-futered water

Molytodenum

Mvinstic acrd

N.(4. Chlorophenvi) -3-phenvil.2-ptupenamide

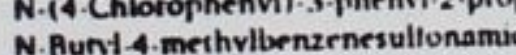

N. Aunts 4. Buryt ether

N.Ethvi-2 propenamicte

N.Ethykyriohe xvlamine

N.Methyl.N-nitroweniline

1aper 1001
6.24

Toot Name (Anaiyto;

ACCEPTABLE ENTRIES: (Cont)

lempinpulamine

isuprowethent
Isaquinatine

ketoendin

-

Lonthanum 190

Laurk acid

lead

Lead 211

Lead 214

Lead snphinate

Lewistle

Lewisisie o

Lindane
Lipids, pereentage

Lithium

m.Xylene

Mognesium
Malathion

Malononitrile

Manganese

Mangance
MCPA
MCPP
Melamin

Mercury

Merrury extractable

Mercury to

Merphos

Methane

Methanol

Methoryctlor

Mechyl-2-heptanols

Methyl-2-heptanon

Methyl sodehyde

Methyl ansonic acid

204

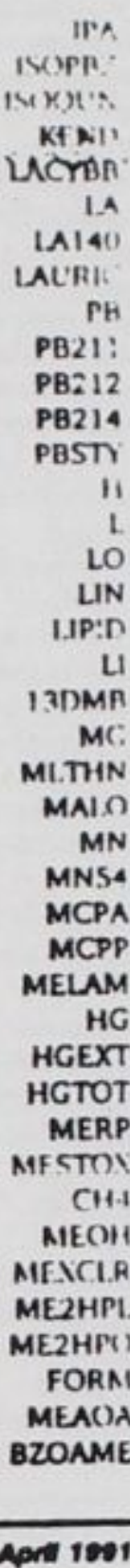

1 apd 1001

2.24

Teet Namo (Anelyno)

ACCEPTABLE ENTAIES: (COML)

N.Methyleniline

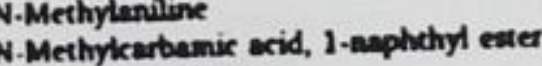

N-Nitrodilherylemine

N. Nirtowo-4hydromprotine

N.Nirrocodi-N-propylemin

N. Nitrosodinamethylamine

N.-Nirrosodiphenthenise

N. Nitroosopentyendidine

N.-Aertanide

N.4.-Dimethrlbeasenesullonamide

N.N.Bis(2-hydroryechyl)dodecane

N.N.Dimethyl 1,2 ethanedianing

N.N.4.Tnmethylbenzenesultonamide

Naphithalene

Naphehoquinon

Neodymium

Neopenirta

Nikkel 63

Niotium

Nitramine
Nurtate

Nintrate
Nitrite

Nitrite, witrate - nonspecifix

Nitroeromatic

Nitrobenzene

Nitroerellulose

Nirrocelluboese $120 \mathrm{~N}$

Nitrogen by kieldehl Method

Nirogen dioxide

Merrogluererine

anes 
Toet Name (Analye)

.24

aCCEPTABle ENTRIES: (Cont)

Nutursodt.N. propviamune

Nenaderane

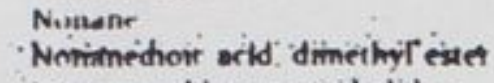

Nonpurgerable or kamir hatides

Nonyt phenol (ani isomer)

Nentievrlane

o.Chlorobrenzaldehyde

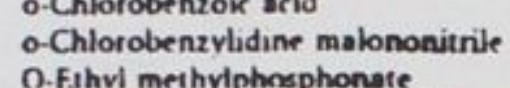

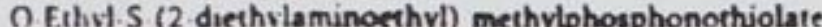

O.Ethyl.S. (2-dusopiopylominoethy) methylphosphonotholate

o-Xviene

Oriadecamethyknriononasiloxane

Ortadecane

Oriaderanoik acid. (2-phenvl-1.3-dioxolan-4.v1) methvil ester

Oriaderanoic acrid. burvi ester

Octadecanoic acid. ethvi ester

Ortaderanoik scid, methyl ester

Ot inderanoic acid. ortaderyl ester
Oriamethvikylotetrasiloxane

Oriane

Octanedioic acid. dimethyl ester

Octanoic acid, methyl ester

Odil \& greas

Organophosphates

Orihophosphate

Oxacyclononane

Oxalk Arid

Oxyzen

p. Chiorophenyimethyl sulfide

p-Chlorophenylmethyl sulfone

p-Chlorophenylmethyl sulfoxide

P-Cymene

p-Divethylaminoazobenzene

p-Tolvenesulifonix axid, heppyl eser

140at 1901

8.2

Teat Neme (Analyte)

ACCEPTABLE ENTRIES: (Com.)

Phenylaretic acid

Phorate
Phosgene .

Phoogene. ort?

Phosphoric arid
Phosphoric acid. diethyt 4 nittophenyt ester
Phosphune acid. ocritliphenyl ester

Phosphune acid. ornidiphenyl este

Phosphorr acud. trnphenit ester

Phosphores
Phithalates

Phithalie arrid

PICLOPAN

Ficic arid

Pinarolit

Plutonium 238 isotope
Plutonium 230 isotope

Polvdimethyl siloxane

Polvnucleat atomatic hidrocatbons

Posassium

Potassium

Pisnated

Promelon
Pronamide

Pronamide
Propanoir acid. 2-hydroxideryl ester

Piopanoir acid, 2-methibutyl erier

Piopanoir arvi. methyl estel

Propyl methytphosphoaic acid

Propyllienzene

Propylene oxide

Protartinium 2.4

Protherphos

Purgealite organic halogen

Prene

Pyridine

IAport 1001
8.24

Tost Name Analye

ACCEPTABLE ENTRIES: (Cont)

Prome
ralmuse and

Falation"

I'aratheon methy!

Aurtivilate ming
PCB 1016

PCB 1016
PCH 1221

PCB 1232
PCA 1242
PCB 1248

PCB 1248
PCB 1254

PCB 1260

Pentinchlorobenzene

Pentachlorobiphenyls

Pentachloroethane

Pentachloronitrobenzen

Pentachioroping

Pentacosane

Pentadecanoic acid

Pentaernhritol tetranitrate

Pentafuorophenol

Pentane

Pentanoik axid
Pentanoix acid, 2-methylburyl eater

Pentanoic acid. 2 -

Pentatriecontas

Pervilene.D12

Petroleum distillares

$\mathrm{PH}$

$\mathrm{pH}$ as lessed in the treld

Phenacetin

Phenanthrenene-D10

Phenol

Phenot D6

Phenolics - nonspecific

Phenoryacetic acid

$413 x+4$

cin

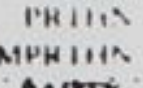

PCBOI

PCB 23

PCB24:

PCB2SA

PCB260

CLSBP

PCH

PCNB

2.240

140 all 1001

Toet Mamo (Analyto)

ACCEPTABLE ENTRIES: (Comt)

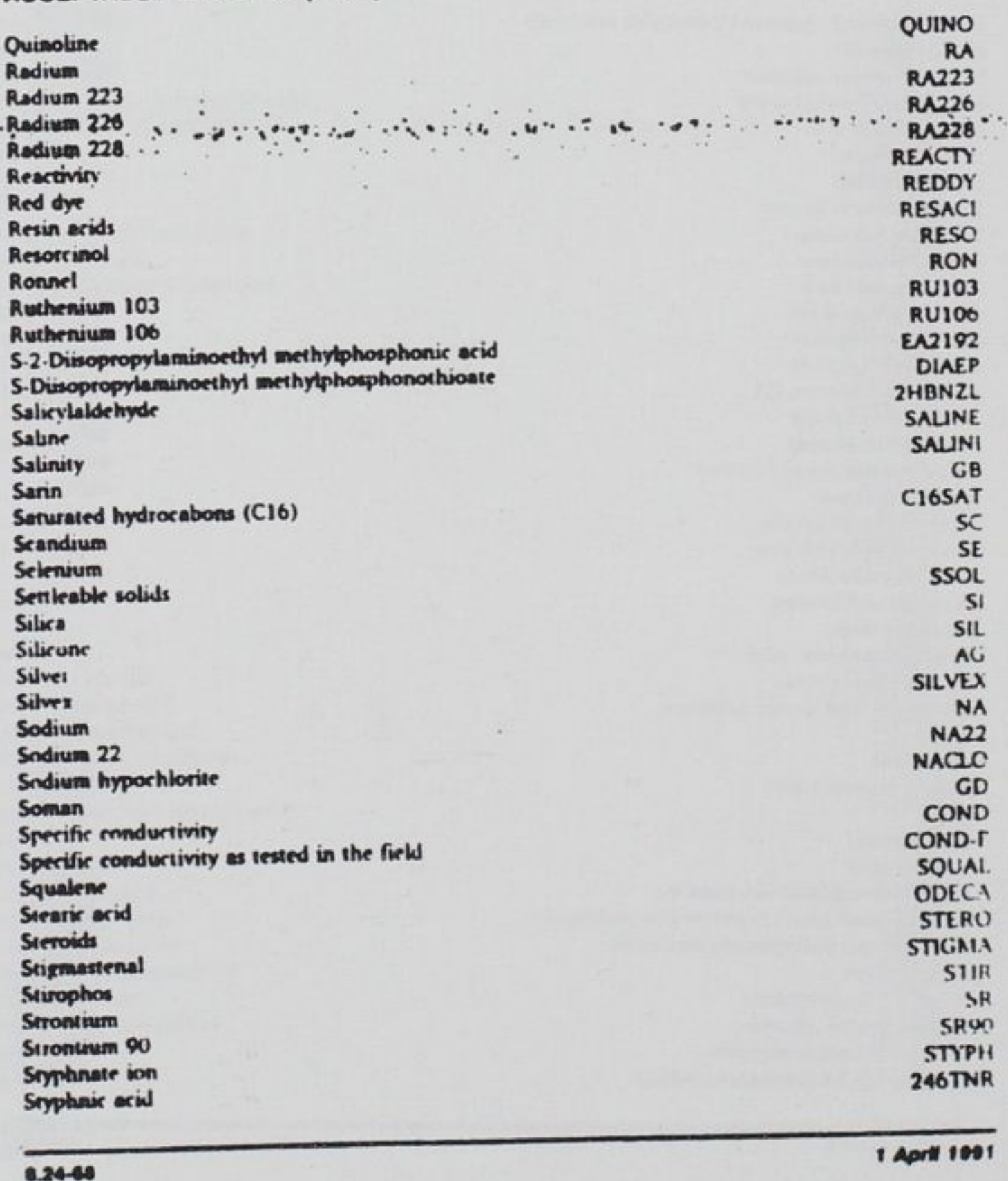




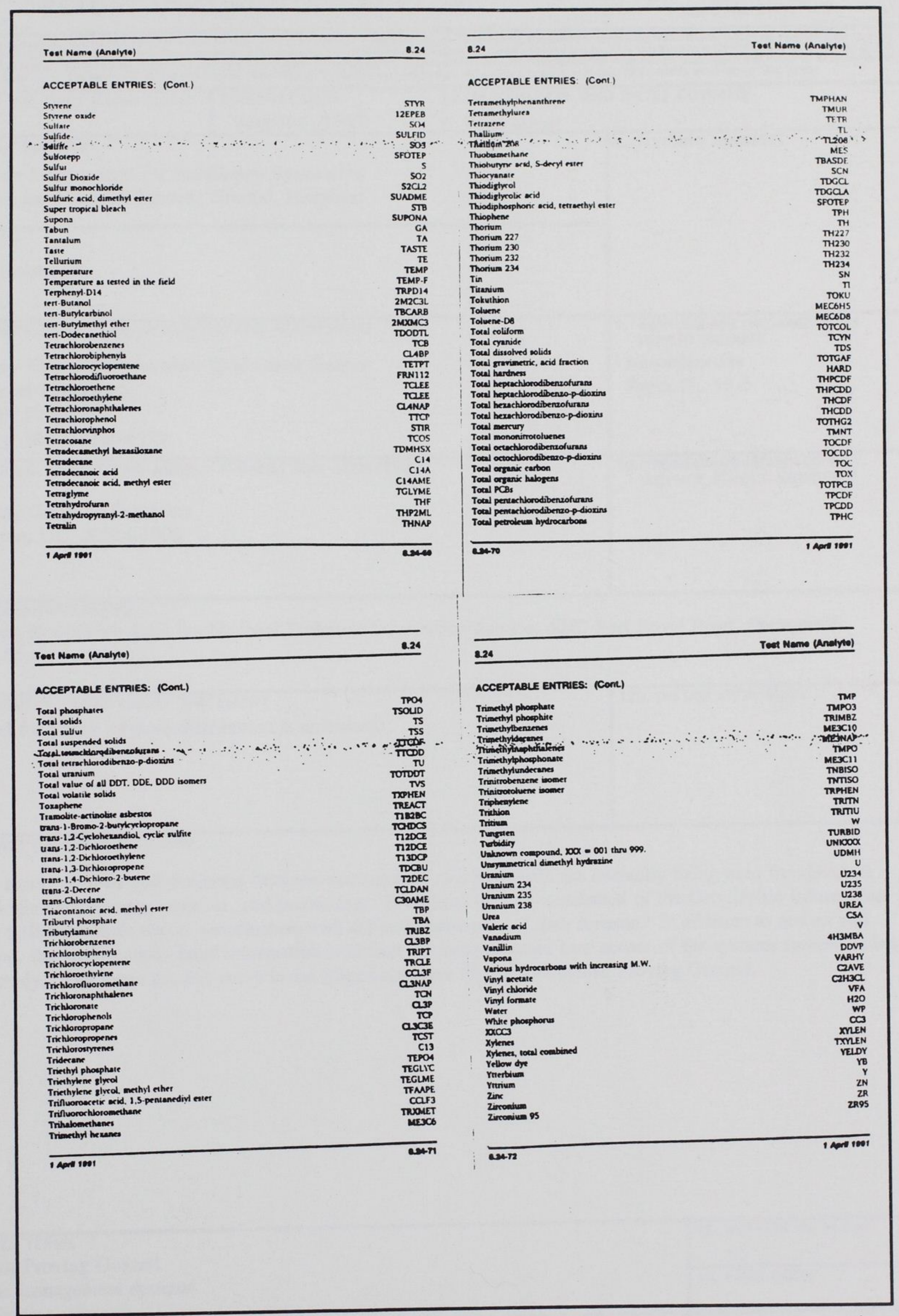


Public reporting burden for this collection of information is estimated to average 1 hour per response. including the time for reviewing instructions, searching existing data sources. gathering and maintaining the data needed, and completing and reviewing the collection of information. Send comments regarding this burden estimate or any other aspect of this collection of information, including suggestions for reducing this burden, to Washington Headquarters Services, Directorate for information Operations and Reports, 1215 Jefferson Davis Highway, Suite 1204. Arlington, VA 22202-4302, and to the Office of Management and Budget, Paperwork Reduction Project (0704-0188), Washington, DC 20503.

\begin{tabular}{|l|l|l|}
\hline 1. AGENCY USE ONLY (Leave blank) & $\begin{array}{l}\text { 2. REPORT DATE } \\
\text { August } 1993\end{array}$ & $\begin{array}{l}\text { 3. REPORT TYPE AND DATES COVERED } \\
\text { Final report }\end{array}$ \\
\hline
\end{tabular}

\section{TITLE AND SUBTITLE} August 1993

5. FUNDING NUMBERS

Information Management for Installation Restoration

with Focus on Aberdeen Proving Ground, Maryland

\section{AUTHOR(S)}

Joe D. Manous, Jr.

\section{PERFORMING ORGANIZATION NAME(S) AND ADDRESS(ES)}

8. PERFORMING ORGANIZATION REPORT NUMBER

U.S. Army Engineer Waterways Experiment Station

Geotechnical Laboratory

Miscellaneous

3909 Halls Ferry Road

Paper GL-93-3

Vicksburg, MS 39180-6199

9. SPONSORING /MONITORING AGENCY NAME(S) AND ADDRESS(ES)

U.S. Army Corps of Engineers

Washington, DC 20314-1000

10. SPONSORING / MONITORING AGENCY REPORT NUMBER

\section{SUPPLEMENTARY NOTES}

This report is available form the National Technical Information Service, 5285 Port Royal Road, Springfield, VA 22161.

\section{2a. DISTRIBUTION/AVAILABILITY STATEMENT}

Approved for public release; distribution is unlimited.

12b. DISTRIBUTION CODE

\section{ABSTRACT (Maximum 200 words)}

This report reviews and evaluates database management systems which are currently being used for chemical and geologic data storage, retrieval, and processing. A review was also conducted of the Geographic Information Systems (GIS) and their use in coordination with different database and data formats. In addition to review and evaluation, the study consolidated information sufficient for inexperienced user access of the systems recommended by this study. The focus for this study is the Edgewood Area (EA) of Aberdeen Proving Ground.

\section{SUBJECT TERMS}

Aberdeen Proving Ground

Database management systems

Geologic data storage

\section{SECURITY CLASSIFICATION OF REPORT}

18

8. SECURITY CLASSIFICATION OF THIS PAGE

UNCLASSIFIED
15. NUMBER OF PAGES

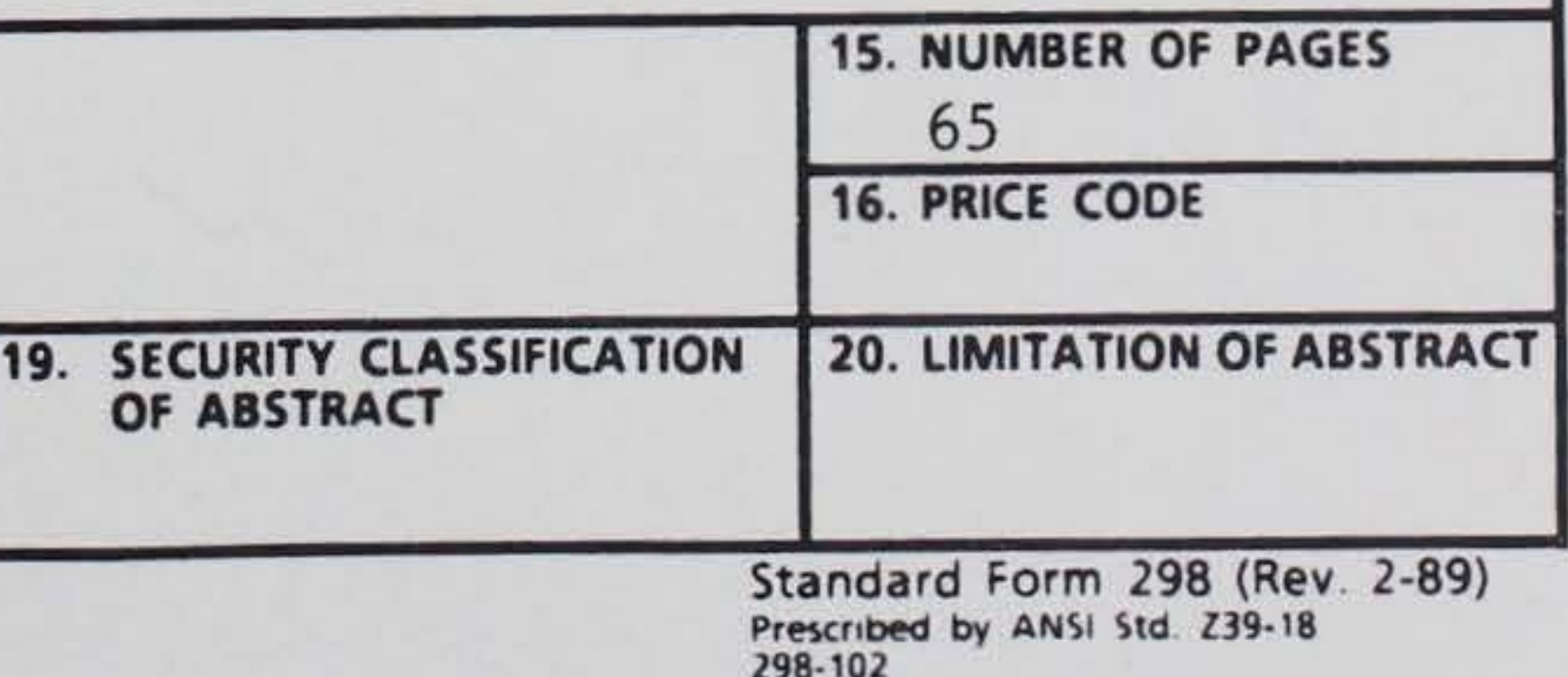

